\title{
Heat Capacity and Bond Dissociation Energy Calculations of Some Fluorinated Ethanol's and its Radicals: $\mathrm{CH}_{3-\mathrm{x}} \mathrm{CH}_{2} \mathrm{~F}_{\mathrm{x}} \mathrm{OH}, \mathrm{CH}_{3} \mathrm{CH}_{2-\mathrm{x}} \mathrm{F}_{\mathrm{x}} \mathrm{OH}$
}

\author{
Hebah M. Abdel-Wahab, Joseph W. Bozzelli \\ Department of Chemistry and Environmental Science, New Jersey Institute of Technology, Newark, New Jersey, USA \\ Email: dr.heathera@gmail.com
}

How to cite this paper: Abdel-Wahab, H.M. and Bozzelli, J.W. (2021) Heat Capacity and Bond Dissociation Energy Calculations of Some Fluorinated Ethanol's and its Radicals: $\mathrm{CH}_{3-\mathrm{x}} \mathrm{CH}_{2} \mathrm{~F}_{\mathrm{x}} \mathrm{OH}, \mathrm{CH}_{3} \mathrm{CH}_{2-\mathrm{x}} \mathrm{F}_{\mathrm{x}} \mathrm{OH}$. Open Journal of Physical Chemistry, 11, 13-53.

https://doi.org/10.4236/ojpc.2021.112002

Received: October 26, 2020

Accepted: March 22, 2021

Published: March 25, 2021

Copyright $\odot 2021$ by author(s) and Scientific Research Publishing Inc. This work is licensed under the Creative Commons Attribution International License (CC BY 4.0).

http://creativecommons.org/licenses/by/4.0/

(c) (i) Open Access

\begin{abstract}
Structures and thermochemical properties of these species were determined by the gaussian M-062x/6-31 $+\mathrm{g}(\mathrm{d}, \mathrm{p})$ calculation enthalpies of formation for 19 fluorinated ethanol and some radicals were calculated with a popular $A b$ initio and density functional theory methods: The gaussian M-062x/6-31 $+\mathrm{g}(\mathrm{d}, \mathrm{p})$ via several series of isodesmic reactions. Entropies $\left(\mathrm{S} 298^{\circ} \mathrm{K}\right.$ in $\mathrm{Cal} \cdot \mathrm{Mol}^{-1} \mathrm{~K}^{-1}$ ) were estimated using the $\mathrm{M}-062 \mathrm{x} / 6-31+\mathrm{g}(\mathrm{d}, \mathrm{p})$ computed frequencies and geometries. Contributions of entropy, $\mathrm{S} 298^{\circ} \mathrm{K}$, and heat capacities, $\mathrm{Cp}(\mathrm{T})$ due to vibration, translation, and external rotation of the molecules were calculated based on the vibration frequencies and structures obtained from the M-062x/6-31 + g (d, p) Density Functional Method. Potential barriers are calculated using $M-062 x / 6-31+g(d, p)$ density functional method and are used to calculate rotor contributions to entropy and heat capacity using integration over energy levels of rotational potential. Rotational barriers were determined and hindered internal rotational contributions for $\mathrm{S} 298^{\circ}-1500^{\circ} \mathrm{K}$, and $\mathrm{Cp}(\mathrm{T})$ were calculated using the rigid rotor harmonic oscillator approximation, with direct integration over energy levels of the intramolecular rotation potential energy curves. Thermochemical properties of fluorinated alcohols are needed for understanding their stability and reactions in the environment and in thermal processes.
\end{abstract}

\section{Keywords}

Thermo-Chemistry, Enthalpy, Fluorinated Ethanol's, Thermochemical Properties

\section{Introduction}

Fluorinated hydrocarbons are used as refrigerants, in polymers, heat exchange fluids, and as solvents. They are present in the atmosphere, lithosphere, and hy- 
drosphere. Because of their less adverse effects on the stratospheric ozone layer, they are used in place of greenhouse gases [1]. Fluorinated hydrocarbons exist as compounds ranging from pure to oxidized intermediates resulting from oxidation in the environment. In order to study their reactivity in biological systems, lifetimes, and in the environment, it's critical to understand the chemical and thermodynamic properties of fluorocarbons and their breakdown intermediates.

The thermochemistry of fluorinated alcohols with one carbon atom was studied in the past and is in the literature [2]. In 2016, Hang Wang studied thermodynamic properties of fluorinated methanol using CBS-QB3, M06, M06-2X, WB97X, W1U, B3LYP, CBS-APNO and G4 Calculations. Small standard deviation suggests good error cancellation of work reactions and accuracy. M06-2x/6-31 $+\mathrm{g}(\mathrm{d}, \mathrm{p})$ calculation had small values for standard deviations. It is an accurate method to calculate Enthalpy of fluorinated alcohols. It shows the second smallest standard deviation after CBS-QB3 method of calculation.

Halogenated compounds are highly stable, have low reactivity and are valued chemicals in industry [3]. Due to its widespread use and their persistence in the environment, they are of concern to the environment. In order to understand the oxidation and reduction reactions involving such molecules, their thermochemical properties must be studied [3].

\subsection{Isodesmic and Isogyric Reaction}

The enthalpy of formation of mono and di fluorinated ethanol's and its radicals has been calculated using Gaussian M-062x/6-31 + g (d, p) method of calculation. In order to calculate the enthalpy of formation of fluorinated ethanols using this method, we use the calculated enthalpies of formation in work reactions along with reference species.

The number of each type of bond must be conserved in the isodesmic reactions in order to cancel any systematic error in the molecular orbital calculations using this method. Calculations of enthalpies of formation are allowed to accuracies close to experimental values by the careful choice of the isodesmic reactions [2]. Standard enthalpy of formation for the reference species used in the isodesmic work reactions along with their uncertainties is listed in Table 1. Taking 1-fluoroethanol as an example, two isodesmic reactions (Table 2) are selected to determine the $\Delta_{\mathrm{f}} \mathrm{H}_{\left(298^{\circ} \mathrm{K}\right)}^{\circ}$ of the target molecule, 1-fluoroethanol. Since the $\Delta_{\mathrm{f}} \mathrm{H}_{\left(298^{\circ} \mathrm{K}\right)}^{\circ}$ values of all species but 1-fluoroethanol in 1 - 2 (Table 2) are known, the $\Delta_{\mathrm{f}} \mathrm{H}_{\left(298^{\circ} \mathrm{K}\right)}$ of the target species 1-fluoroethanol is obtained from this data and the calculated $\Delta_{\mathrm{rxn}} \mathrm{H}_{\left(298^{\circ} \mathrm{K}\right)}^{\circ} \cdot \Delta_{\mathrm{f}} \mathrm{H}_{\left(298^{\circ} \mathrm{K}\right)}^{\circ}$ calculated using two different reference molecules are within $\pm 2 \mathrm{Kcal} \cdot \mathrm{mol}^{-1}$.

\subsection{Reference Species}

Standard enthalpy of formation for the reference species used in the isodesmic work reactions along with their uncertainties is listed in Table 1. Table 2 provides one example on the method of Isodesmic Work Reactions used for the 
Table 1. Reference Species in the Isodesmic Reactions Standard Enthalpy of Formation Values $\left(\mathrm{kcal} \cdot \mathrm{mol}^{-1}\right)[4]$.

\begin{tabular}{|c|c|c|c|}
\hline Species & $\Delta_{\mathrm{f}} \mathrm{H}^{\circ}{ }_{\left(298^{\circ} \mathrm{K}\right)}$ & Species & $\Delta_{\mathrm{f}} \mathrm{H}^{\circ}{ }_{\left(298^{*} \mathrm{~K}\right)}$ \\
\hline \multirow{2}{*}{$\mathrm{CH}_{3} \mathrm{~F}$} & $-56.54 \pm 0.07^{\mathrm{a}}$ & $\mathrm{CH}_{3} \mathrm{OOH}$ & $-30.96 \pm 0.67^{\mathrm{b}}$ \\
\hline & $-56.62 \pm 0.48^{\mathrm{h}}$ & $\mathrm{CH}_{3} \mathrm{CH}_{2} \mathrm{OOH}$ & $-38.94 \pm 0.81^{\mathrm{b}}$ \\
\hline $\mathrm{CH}_{3} \mathrm{CH}_{2} \mathrm{~F}$ & $-65.42 \pm 1.11^{\mathrm{a}}$ & $\mathrm{CH}_{3} \mathrm{CH}_{2} \mathrm{CH}_{2} \mathrm{OOH}$ & $-44.03 \pm 0.67^{\mathrm{b}}$ \\
\hline $\mathrm{CH}_{3} \mathrm{CH}_{2} \mathrm{CH}_{2} \mathrm{~F}$ & $-70.24 \pm 1.30^{\mathrm{a}}$ & $\mathrm{CH}_{3} \mathrm{OO}$ & $2.37 \pm 1.24^{\mathrm{b}}$ \\
\hline \multirow{2}{*}{$\mathrm{CH}_{2} \mathrm{~F}_{2}$} & $-108.07 \pm 1.46^{\mathrm{a}}$ & \multirow{2}{*}{$\mathrm{CH}_{3} \mathrm{CH}_{2} \mathrm{OO} \cdot \mathrm{CH}_{3} \mathrm{CH}_{2} \mathrm{CH}_{2} \mathrm{OO} \cdot$} & $-6.19 \pm 0.92^{b}$ \\
\hline & $-107.67 \pm 0.48^{\mathrm{h}}$ & & $-11.35 \pm 1.24^{\mathrm{b}}$ \\
\hline $\mathrm{CH}_{3} \mathrm{CHF}_{2}$ & $-120.87 \pm 1.62^{\mathrm{a}}$ & $\mathrm{CH}_{4}$ & $-17.81 \pm 0.01^{\mathrm{c}}$ \\
\hline $\mathrm{CH}_{3} \mathrm{CH}_{2} \mathrm{CHF}_{2}$ & $-125.82 \pm 1.65^{\mathrm{a}}$ & $\mathrm{CH}_{3} \mathrm{CH}_{3}$ & $-20.05 \pm 0.04^{\mathrm{c}}$ \\
\hline \multirow{2}{*}{$\mathrm{CHF}_{3}$} & $-166.71 \pm 1.97^{\mathrm{h}}$ & \multirow[b]{2}{*}{$\mathrm{CH}_{3} \mathrm{CH}_{2} \mathrm{CH}_{3} \mathrm{CH}_{3} \mathrm{CH}_{2} \mathrm{CH}_{2} \mathrm{CH}_{3}$} & $-25.01 \pm 0.06^{\mathrm{i}}$ \\
\hline & $-166.09 \pm 0.48^{\mathrm{h}}$ & & $-30.07 \pm 0.08^{\mathrm{i}}$ \\
\hline $\mathrm{CH}_{3} \mathrm{CF}_{3}$ & $-180.51 \pm 2.05^{\mathrm{a}}$ & $\mathrm{CH}_{3} \mathrm{O}^{\cdot}$ & $5.15 \pm 0.08^{c}$ \\
\hline $\mathrm{CH}_{3} \mathrm{CH}_{2} \mathrm{CF}_{3}$ & $-185.48 \pm 2.15^{\mathrm{a}}$ & $\mathrm{CH}_{3} \mathrm{CH}_{2} \mathrm{O}^{\bullet}$ & $-3.01^{\mathrm{d}}$ \\
\hline $\mathrm{CH}_{3} \cdot$ & $34.98 \pm 0.02^{c}$ & $\mathrm{OH}$ & $8.96 \pm 0.01^{c}$ \\
\hline $\mathrm{CH}_{3} \mathrm{CH}_{2} \cdot$ & $28.65 \pm 0.07^{\mathrm{c}}$ & $\mathrm{CH}_{3} \mathrm{OH}$ & $-47.97 \pm 0.04^{\mathrm{c}}$ \\
\hline $\mathrm{CH}_{3} \mathrm{CH}_{2} \mathrm{CH}_{2}^{\cdot}$ & $\begin{array}{c}24.21 \pm 0.24^{\mathrm{gj}} \\
24.18^{\mathrm{i}}\end{array}$ & $\mathrm{CH}_{3} \mathrm{CH}_{2} \mathrm{OH}$ & $-56.07 \pm 0.05^{\mathrm{i}}$ \\
\hline $\mathrm{H}$ & $52.10^{c}$ & HOO & $2.94^{\mathrm{cj}}$ \\
\hline $\mathrm{O}$ & $59.57^{\mathrm{c}}$ & $\mathrm{HOOH}$ & $\begin{array}{c}-32.39 \pm 0.04^{\mathrm{fj}} \\
-32.37^{\mathrm{i}}\end{array}$ \\
\hline
\end{tabular}

${ }^{a}$ Wang [2], ${ }^{b}$ Wang [4], 'Ruscic [5], ${ }^{\mathrm{d} B u r k e ~[6], ~}{ }^{\mathrm{e}}$ Chase [7], fLuo [8], ${ }^{\mathrm{B}}$ Bodi [9], ${ }^{\mathrm{h}}$ Pedley, ${ }^{\mathrm{i}} \mathrm{ATcT}$ Tables [10], ${ }^{\mathrm{h}}$ Csontos [11]. .The value we used in this study [4]

Table 2. Example for Enthalpy of Formation Calculations for 1-fluoroethanol using Isodesmic Reactions; using different reference molecules, Units in $\mathrm{kcal} \cdot \mathrm{mol}^{-1}$.

\begin{tabular}{|c|c|c|c|c|c|}
\hline Isodesmic Reactions & $\begin{array}{c}\Delta \mathrm{H}_{\mathrm{Rxn}(298 \cdot \mathrm{K})}^{\circ} \\
\text { Hartrees }\end{array}$ & $\begin{array}{c}\Delta \mathrm{H}_{\mathrm{Rxn}(298 \cdot \mathrm{K})} \\
\mathrm{kcal} / \mathrm{mol}^{-1}\end{array}$ & $\begin{array}{c}\Delta_{\mathrm{f}} \mathrm{H}^{\circ}{ }_{\left(298^{\circ} \mathrm{K}\right)} \mathrm{CH}_{3-\mathrm{x}} \mathrm{CH}_{2} \mathrm{~F}_{\mathrm{x}} \mathrm{OH} \\
\mathrm{CH}_{3} \mathrm{CH}_{2-\mathrm{x}} \mathrm{F}_{\mathrm{x}} \mathrm{OH} \mathrm{kcal} \cdot \mathrm{mol}^{-1}\end{array}$ & $\begin{array}{c}\text { Error } \\
\mathrm{kcal} \cdot \mathrm{mol}^{-1}\end{array}$ & $\begin{array}{c}\text { Equ. } \\
\#\end{array}$ \\
\hline $\begin{array}{c}\mathrm{CH}_{2} \mathrm{FCH}_{2} \mathrm{OH}+\mathrm{CH}_{4}=\mathrm{CH}_{3} \mathrm{CH}_{2} \mathrm{OH}+\mathrm{CH}_{3} \mathrm{~F} \\
-254.173115^{\mathrm{a}}-40.447961-154.926666-139.683801 \\
\text { Reference Values }-17.81-56.21-56.54 \\
\mathrm{Kcal} \cdot \mathrm{mol}^{-1}\end{array}$ & 0.010609 & 6.657243 & -101.6 & \pm 0.2 & 1 \\
\hline $\begin{array}{c}\mathrm{CH}_{2} \mathrm{FCH}_{2} \mathrm{OH}+\mathrm{CH}_{3} \mathrm{CH}_{3}=\mathrm{CH}_{3} \mathrm{CH}_{2} \mathrm{OH}+\mathrm{CH}_{3} \mathrm{CH}_{2} \mathrm{~F} \\
-254.173115-79.717768-154.926666-178.963776 \\
-20.05-56.21-65.42\end{array}$ & 0.000441 & 0.276731 & -101.9 & \pm 1.3 & 2 \\
\hline Reported $\Delta_{\mathrm{f}} \mathrm{H}^{\circ}(298) \mathrm{kcal} \cdot \mathrm{mol}^{-1}$ & & & $-101.7 \pm 0.7$ & & \\
\hline Standard Deviation over rxns & & & \pm 0.1 & & \\
\hline
\end{tabular}

${ }^{\mathrm{a}}$ Hartrees, $\mathrm{kcal} \cdot \mathrm{mol}^{-1}$; ${ }^{\star S D}$ Standard Deviation $\mathrm{kcal} \cdot \mathrm{mol}^{-1}$ Errors reported avg of sum of uncertainties in rxn's reference species.

calculation of the Standard Enthalpy of Formation $\Delta_{\mathrm{f}} \mathrm{H}_{\left(298^{\circ} \mathrm{K}\right)}$ for the fluoroethanols.

\subsection{Computational Method}

Composite calculations and series of Isodesmic Reactions are used to calculate 
enthalpy of formation of fluorinated ethanols. All calculations are performed using the Gaussian 16 program. The DFT method M06-2x is used to initially analyze optimized structures, frequencies, thermo energies and internal rotors of the molecules studied. It's a Global-hybrid meta-GGA density functional approximation, GGA, generalized gradient approximation, in which the density functional depends on the up and down spin densities and their reduced gradient, meta GGA, in which the functional also depends on the up and down spin kinetic energy densities, hybrid GGA, a combination of GGA with Hartree-Fock exchange, hybrid meta GGA, a combination of meta GGA with Hartree-Fock exchange [12]. All reported values are for standard state of $298 \mathrm{~K}$ and $1 \mathrm{~atm}$. We continue the calculation of fluorinated alcohols in this study with this method, because the $\mathrm{M}-062 \mathrm{x} / 6-31+\mathrm{g}(\mathrm{d}, \mathrm{p})$ level of calculation have been applied to fluoro hydrocarbons 6 with small reported standard deviations values.

\section{Results and Discussion}

\subsection{Entropy and Heat Capacity Values}

Internal rotor contributions to calculated entropy and heat capacity at 298 - 1500 $\mathrm{K}$ were determined using the molecular mass of each molecule, number of optical isomers, symmetry of the molecule, electron degeneracy, moment of inertia, and vibrational frequencies values (Table 3). The vibrational frequencies for the calculation of heat capacity and entropy at the M-062x/6-31 + g (d, p) level of calculation was scaled by a factor of 0.97 [13]. The moment of inertia values is shown in the Supplemental Information Table provided. To calculate the contributions of external rotor, vibration and transition to the calculated entropy and heat capacity, the "SMCPS" [14] program is used. It employs the rigid-rotor harmonic oscillator approximation using moment of inertia from optimized structure and frequencies. The "Rotator" [15] program by Lay et al. [16] is used to calculate internal rotor contributions from the corresponding internal rotor torsion frequencies. In this paper, a torsional potential curve presenting a ten-parameter Fourier series function is used to calculate the contribution of internal rotor. Parameters and detailed functions are shown in the Supplemental Information Table provided. Rotor [16] program is used to calculate thermodynamic functions from hindered rotations with arbitrary potentials.

Calculation of the Hamiltonian matrix of the internal rotor, and subsequent calculation of energy levels by direct diagonalization of the matrix are employed by this technique. Rotational barriers versus dihedral angle are presented as a potential curve. In this paper, the calculated torsional potential at discrete torsional angles.

$$
V(\Phi)=a_{0}+\sum a_{i} \cos (i \Phi)+\sum b_{j} \cos (j \Phi) \quad i, j=1-10
$$

The coefficients $a_{i}$ and $b_{j}$ are calculated to present the maxima and minima of the torsional potentials with a possibility to shift from the extreme angular positions. 
Table 3. Monofluoro and Difluoro-Ethanol's Ideal Gas phase Entropy and Heat Capacity obtained using M-062x/6-31 + g (d, p) level of theory $\left(\mathrm{Cal} \cdot \mathrm{mol}^{-1} \cdot \mathrm{K}^{-1}\right)$.

\begin{tabular}{|c|c|c|c|c|c|c|c|c|c|}
\hline Species & & $S\left(298^{\circ} \mathrm{K}\right)$ & $\mathrm{Cp}\left(298^{\circ} \mathrm{K}\right)$ & $\mathrm{Cp}\left(400^{\circ} \mathrm{K}\right)$ & $\mathrm{Cp}\left(500^{\circ} \mathrm{K}\right)$ & $\mathrm{Cp}\left(600^{\circ} \mathrm{K}\right)$ & $\mathrm{Cp}\left(800^{\circ} \mathrm{K}\right)$ & $\mathrm{Cp}\left(1000^{\circ} \mathrm{K}\right)$ & $\mathrm{Cp}\left(1500^{\circ} \mathrm{K}\right)$ \\
\hline \multirow{4}{*}{$\mathrm{CH}_{2} \mathrm{FCH}_{2} \mathrm{OH}$} & \multirow{4}{*}{$\begin{array}{c}\text { TVR } \\
\text { Internal Rotor } \\
\text { Total }\end{array}$} & 64.74 & 13.23 & 17.12 & 20.72 & 23.75 & 28.18 & 31.11 & 35.06 \\
\hline & & 3.84 & 3.24 & 3.48 & 3.43 & 3.22 & 2.68 & 2.23 & 1.62 \\
\hline & & 2.02 & 3.15 & 3.33 & 3.04 & 2.66 & 2.05 & 1.69 & 1.30 \\
\hline & & 70.60 & 19.62 & 23.94 & 27.20 & 29.62 & 32.91 & 35.03 & 37.98 \\
\hline \multirow{4}{*}{$\mathrm{CH}_{3} \mathrm{CHFOH}$} & \multirow{4}{*}{$\begin{array}{c}\text { TVR } \\
\text { Internal Rotor } \\
\text { Total }\end{array}$} & 65.17 & 14.06 & 17.90 & 21.37 & 24.26 & 28.51 & 31.32 & 35.15 \\
\hline & & 4.48 & 2.13 & 2.09 & 1.95 & 1.80 & 1.56 & 1.40 & 1.19 \\
\hline & & 2.67 & 2.17 & 2.21 & 2.18 & 2.09 & 1.89 & 1.71 & 1.40 \\
\hline & & 72.32 & 18.35 & 22.21 & 25.50 & 28.16 & 31.96 & 34.42 & 37.75 \\
\hline \multirow{4}{*}{$\mathrm{C} \cdot \mathrm{HFCH}_{2} \mathrm{OH}$} & \multirow{4}{*}{$\begin{array}{c}\text { TVR } \\
\text { Internal Rotor } \\
\text { Total }\end{array}$} & 66.30 & 13.54 & 16.96 & 19.97 & 22.48 & 26.29 & 29.04 & 33.33 \\
\hline & & 6.19 & 2.31 & 1.91 & 1.69 & 1.54 & 1.36 & 1.25 & 1.13 \\
\hline & & 3.09 & 3.16 & 2.52 & 2.04 & 1.74 & 1.41 & 1.25 & 1.11 \\
\hline & & 75.58 & 19.01 & 21.38 & 23.70 & 25.76 & 29.06 & 31.55 & 35.56 \\
\hline \multirow{4}{*}{$\mathrm{CH}_{2} \mathrm{FCH} \bullet \mathrm{OH}$} & \multirow{4}{*}{$\begin{array}{c}\text { TVR } \\
\text { Internal Rotor } \\
\text { Total }\end{array}$} & 66.47 & 13.76 & 17.09 & 20.04 & 22.52 & 26.29 & 29.02 & 33.31 \\
\hline & & 5.72 & 2.34 & 2.22 & 2.09 & 1.95 & 1.73 & 1.56 & 1.32 \\
\hline & & 6.00 & 1.96 & 1.95 & 1.95 & 1.91 & 1.79 & 1.65 & 1.40 \\
\hline & & 78.19 & 18.06 & 21.27 & 24.07 & 26.38 & 29.81 & 32.24 & 36.03 \\
\hline \multirow{3}{*}{$\mathrm{CH}_{2} \mathrm{FCH}_{2} \mathrm{O} \bullet$} & TVR & 75.30 & 20.44 & 23.66 & 26.27 & 28.39 & 31.55 & 33.79 & 37.16 \\
\hline & Internal Rotor & 6.08 & 2.20 & 1.96 & 1.7982 & 1.68 & 1.53 & 1.43 & 1.27 \\
\hline & Total & 81.38 & 22.64 & 25.62 & 28.07 & 30.07 & 33.08 & 35.21 & 38.42 \\
\hline \multirow{4}{*}{$\mathrm{CH}_{2} \bullet \mathrm{CHFOH}$} & \multirow{4}{*}{$\begin{array}{c}\text { TVR } \\
\text { Internal Rotor } \\
\text { Total }\end{array}$} & 66.89 & 14.80 & 18.21 & 21.07 & 23.40 & 26.90 & 29.44 & 33.49 \\
\hline & & 4.94 & 1.42 & 1.25 & 1.17 & 1.12 & 1.06 & 1.04 & 1.01 \\
\hline & & 2.84 & 1.95 & 2.12 & 2.12 & 2.04 & 1.83 & 1.64 & 1.35 \\
\hline & & 74.67 & 18.17 & 21.58 & 24.35 & 26.55 & 29.79 & 32.11 & 35.86 \\
\hline \multirow{4}{*}{$\mathrm{CH}_{3} \mathrm{CF} \bullet \mathrm{OH}$} & \multirow{4}{*}{$\begin{array}{c}\text { TVR } \\
\text { Internal Rotor } \\
\text { Total }\end{array}$} & 66.56 & 13.94 & 17.25 & 20.17 & 22.62 & 26.38 & 29.11 & 33.38 \\
\hline & & 4.91 & 1.97 & 1.75 & 1.57 & 1.44 & 1.27 & 1.18 & 1.08 \\
\hline & & 1.71 & 2.60 & 3.17 & 3.22 & 3.01 & 2.43 & 1.99 & 1.45 \\
\hline & & 73.18 & 18.50 & 22.17 & 24.96 & 27.07 & 30.08 & 32.28 & 35.92 \\
\hline \multirow{3}{*}{$\mathrm{CH}_{3} \mathrm{CHFO} \bullet$} & TVR & 66.73 & 14.44 & 18.16 & 21.39 & 24.09 & 28.18 & 31.12 & 35.58 \\
\hline & Internal Rotor & 4.55 & 2.12 & 2.05 & 1.90 & 1.75 & 1.51 & 1.36 & 1.18 \\
\hline & Total & 71.27 & 16.56 & 20.21 & 23.29 & 25.84 & 29.69 & 32.48 & 36.75 \\
\hline \multirow{4}{*}{$\mathrm{CH}_{2} \mathrm{FCHFOH}$} & \multirow{4}{*}{$\begin{array}{c}\text { TVR } \\
\text { Internal Rotor } \\
\text { Total }\end{array}$} & 67.46 & 15.41 & 19.51 & 23.12 & 26.07 & 30.23 & 32.83 & 36.15 \\
\hline & & 0.00 & 0.00 & 0.00 & 0.00 & 0.00 & 0.00 & 0.00 & 0.00 \\
\hline & & 6.12 & 3.11 & 2.64 & 2.24 & 1.94 & 1.57 & 1.37 & 1.17 \\
\hline & & 73.58 & 18.52 & 22.15 & 25.35 & 28.00 & 31.79 & 34.20 & 37.31 \\
\hline \multirow{4}{*}{$\mathrm{CF}_{2} \mathrm{HCH}_{2} \mathrm{OH}$} & \multirow{4}{*}{$\begin{array}{c}\text { TVR } \\
\text { Internal Rotor } \\
\text { Total }\end{array}$} & 62.94 & 12.00 & 15.54 & 18.95 & 21.90 & 26.43 & 29.57 & 34.06 \\
\hline & & 4.98 & 3.36 & 3.20 & 2.97 & 2.72 & 2.26 & 1.93 & 1.48 \\
\hline & & 2.23 & 3.21 & 3.17 & 2.81 & 2.44 & 1.91 & 1.60 & 1.27 \\
\hline & & 70.15 & 18.57 & 21.91 & 24.72 & 27.06 & 30.60 & 33.10 & 36.81 \\
\hline
\end{tabular}


Continued

\begin{tabular}{|c|c|c|c|c|c|c|c|c|c|}
\hline \multirow{4}{*}{$\mathrm{CH}_{3} \mathrm{CF}_{2} \mathrm{OH}$} & \multirow{4}{*}{$\begin{array}{c}\text { TVR } \\
\text { Internal Rotor } \\
\text { Total }\end{array}$} & 68.41 & 16.53 & 20.74 & 24.23 & 27.01 & 30.87 & 33.29 & 36.37 \\
\hline & & 4.53 & 2.13 & 2.08 & 1.93 & 1.77 & 1.53 & 1.38 & 1.18 \\
\hline & & 1.58 & 2.45 & 3.09 & 3.28 & 3.14 & 2.60 & 2.14 & 1.53 \\
\hline & & 74.52 & 21.11 & 25.91 & 29.44 & 31.93 & 35.01 & 36.80 & 39.08 \\
\hline \multirow{4}{*}{$\mathrm{CH}_{2} \mathrm{FC} \cdot \mathrm{FOH}$} & \multirow{4}{*}{$\begin{array}{c}\text { TVR } \\
\text { Internal Rotor } \\
\text { Total }\end{array}$} & 69.58 & 15.15 & 18.76 & 21.84 & 24.36 & 28.05 & 30.59 & 34.38 \\
\hline & & 6.18 & 3.28 & 2.51 & 2.07 & 1.80 & 1.50 & 1.34 & 1.16 \\
\hline & & 3.15 & 1.91 & 1.96 & 1.91 & 1.83 & 1.65 & 1.50 & 1.27 \\
\hline & & 78.90 & 20.34 & 23.22 & 25.82 & 27.99 & 31.19 & 33.43 & 36.81 \\
\hline \multirow{4}{*}{$\mathrm{C} \cdot \mathrm{HFCFHOH}$} & \multirow{4}{*}{$\begin{array}{c}\text { TVR } \\
\text { Internal Rotor } \\
\text { Total }\end{array}$} & 69.60 & 15.48 & 19.22 & 22.30 & 24.76 & 28.32 & 30.75 & 34.41 \\
\hline & & 6.92 & 2.03 & 1.69 & 1.49 & 1.37 & 1.22 & 1.15 & 1.07 \\
\hline & & 1.72 & 2.79 & 3.10 & 3.03 & 2.83 & 2.37 & 2.02 & 1.52 \\
\hline & & 78.25 & 20.30 & 24.01 & 26.83 & 28.96 & 31.91 & 33.92 & 37.00 \\
\hline \multirow{3}{*}{$\mathrm{CH}_{2} \mathrm{FCHFO} \bullet$} & TVR & 70.53 & 15.99 & 19.88 & 23.22 & 25.94 & 29.93 & 32.65 & 36.59 \\
\hline & Internal Rotor & 5.48 & 2.92 & 2.79 & 2.58 & 2.37 & 2.01 & 1.76 & 1.40 \\
\hline & Total & 76.01 & 18.91 & 22.67 & 25.80 & 28.31 & 31.94 & 34.41 & 37.99 \\
\hline \multirow{4}{*}{$\mathrm{CHF}_{2} \mathrm{C} \cdot \mathrm{HOH}$} & \multirow{4}{*}{$\begin{array}{c}\text { TVR } \\
\text { Internal Rotor } \\
\text { Total }\end{array}$} & 68.27 & 15.46 & 19.18 & 22.25 & 24.71 & 28.28 & 30.72 & 34.40 \\
\hline & & 6.07 & 2.65 & 2.30 & 2.05 & 1.86 & 1.61 & 1.45 & 1.24 \\
\hline & & 1.51 & 2.50 & 3.06 & 3.18 & 3.06 & 2.60 & 2.20 & 1.61 \\
\hline & & 75.85 & 20.61 & 24.54 & 27.48 & 29.63 & 32.49 & 34.37 & 37.25 \\
\hline \multirow{4}{*}{$\mathrm{C} \cdot \mathrm{F}_{2} \mathrm{CH}_{2} \mathrm{OH}$} & \multirow{4}{*}{$\begin{array}{c}\text { TVR } \\
\text { Internal Rotor } \\
\text { Total }\end{array}$} & 69.57 & 15.16 & 18.73 & 21.81 & 24.32 & 28.03 & 30.58 & 34.37 \\
\hline & & 6.18 & 2.68 & 2.38 & 2.11 & 1.89 & 1.5848 & 1.41 & 1.19 \\
\hline & & 3.46 & 2.45 & 2.04 & 1.75 & 1.55 & 1.32 & 1.21 & 1.09 \\
\hline & & 79.21 & 20.29 & 23.15 & 25.66 & 27.76 & 30.94 & 33.19 & 36.65 \\
\hline \multirow{3}{*}{$\mathrm{CHF}_{2} \mathrm{CH}_{2} \mathrm{O} \bullet$} & TVR & 70.02 & 15.31 & 18.91 & 22.14 & 24.87 & 29.03 & 31.94 & 36.19 \\
\hline & Internal Rotor & 5.78 & 2.51 & 2.41 & 2.26 & 2.10 & 1.83 & 1.63 & 1.34 \\
\hline & Total & 75.79 & 17.82 & 21.32 & 24.40 & 26.97 & 30.86 & 33.57 & 37.53 \\
\hline \multirow{4}{*}{$\mathrm{CH}_{2} \cdot \mathrm{CF}_{2} \mathrm{OH}$} & \multirow{4}{*}{$\begin{array}{c}\text { TVR } \\
\text { Internal Rotor } \\
\text { Total }\end{array}$} & 70.20 & 17.30 & 21.07 & 23.93 & 26.13 & 29.24 & 31.38 & 34.69 \\
\hline & & 4.97 & 1.37 & 1.23 & 1.16 & 1.11 & 1.06 & 1.04 & 1.01 \\
\hline & & 1.78 & 2.81 & 3.33 & 3.25 & 2.9407 & 2.30 & 1.87 & 1.38 \\
\hline & & 76.95 & 21.48 & 25.63 & 28.35 & 27.25 & 32.61 & 34.29 & 37.09 \\
\hline \multirow{4}{*}{$\mathrm{CH}_{3} \mathrm{CF}_{2} \mathrm{O}$ • } & \multirow{4}{*}{$\begin{array}{c}\text { TVR } \\
\text { Internal Rotor } \\
\text { Total }\end{array}$} & 70.14 & 16.85 & 20.78 & 23.99 & 26.55 & 30.30 & 32.87 & 36.66 \\
\hline & & 4.84 & 2.08 & 1.89 & 1.70 & 1.54 & 1.35 & 1.23 & 1.11 \\
\hline & & 5.78 & 2.51 & 2.41 & 2.26 & 2.10 & 1.83 & 1.63 & 1.34 \\
\hline & & 80.75 & 21.44 & 25.08 & 27.94 & 30.20 & 33.47 & 35.73 & 39.11 \\
\hline
\end{tabular}

Calculations of heat capacity and standard entropy based on benchmark database and the computational chemistry comparison for the M-062x/6-31 + g (d, p) calculation method, the vibrational frequencies were scaled by a factor of 0.987 [13]. Potential Energy profiles for mono and di fluorinated ethanol and their related radicals are listed in the Supplemental Information Table provided., the solid lines are the fit of Fourier series expansion, rotator contribution 
for barriers below $7 \mathrm{kcal} \cdot \mathrm{mol}^{-1}$ were added to the SMCPS calculated entropy and heat capacity. Energies are in $\mathrm{kcal} \cdot \mathrm{mol}^{-1}$ (Table 3).

\subsection{Standard Enthalpy Values}

Enthalpies of formation were determined from isodesmic work reactions from M-062x/6-31 + g (d, p) method of calculation. The standard enthalpy of formation for the reference species along with their uncertainties, which are used the isodesmic work reactions, Table 4, are listed in Table $1 \mathrm{in} \mathrm{kcal} \cdot \mathrm{mol}^{-1}$. The standard deviation was calculated [17] for all Enthalpies of formation values for all 19 fluorinated ethanol and is included in Table 4. Details of the method of

Table 4. Standard Enthalpy of Formation using isodesmic reactions: Monofluoro and Difluoro-Ethanols using the M06-2x/6-31 + $\mathrm{g}(\mathrm{d}, \mathrm{p})$ Level of Theory. Errors reported as sum of avg uncertainty in rxn's reference species.

\begin{tabular}{|c|c|c|c|c|c|c|c|}
\hline \multicolumn{4}{|c|}{$\begin{array}{l}\text { Isodesmic Reactions } \\
\text { Target Specie }\end{array}$} & \multirow[t]{2}{*}{$\begin{array}{c}\Delta \mathrm{H}^{\circ}{ }_{\text {Rxn }\left(298^{\circ} \mathrm{K}\right)} \\
\text { Hartrees }\end{array}$} & \multirow[t]{2}{*}{$\begin{array}{l}\Delta \mathrm{H}^{{ }^{\circ}}{ }_{\mathrm{Rxn}\left(298^{\circ} \mathrm{K}\right)} \\
\mathrm{Kcal} / \mathrm{mole}^{1}\end{array}$} & \multirow[t]{2}{*}{$\begin{array}{l}\Delta_{\mathrm{f}} \mathrm{H}^{\circ}{ }_{(298 \cdot \mathrm{K})} \\
\mathrm{kcal} \cdot \mathrm{mol}^{-1}\end{array}$} & \multirow[t]{2}{*}{$\begin{array}{c}\text { Error } \\
\mathrm{kcal} \cdot \mathrm{mol}^{-1}\end{array}$} \\
\hline $\mathrm{CH}_{2} \mathrm{FCH}_{2} \mathrm{OH}$ & $+\mathrm{CH}_{4}=$ & $\mathrm{CH}_{3} \mathrm{CH}_{2} \mathrm{OH}$ & $+\mathrm{CH}_{3} \mathrm{~F}$ & & & & \\
\hline-254.173115 & $\begin{array}{l}-40.447961 \\
-17.81\end{array}$ & $\begin{array}{l}-154.926666 \\
-56.21-56.54\end{array}$ & -139.683801 & 0.010609 & 6.657243 & -101.6 & \pm 0.2 \\
\hline $\begin{array}{l}\mathrm{CH}_{2} \mathrm{FCH}_{2} \mathrm{OH} \\
-254.173115\end{array}$ & $\begin{array}{l}+\mathrm{CH}_{3} \mathrm{CH}_{3}= \\
-79.717768 \\
-20.05\end{array}$ & $\begin{array}{l}\mathrm{CH}_{3} \mathrm{CH}_{2} \mathrm{OH} \\
-154.926666 \\
-56.21\end{array}$ & $\begin{array}{l}+\mathrm{CH}_{3} \mathrm{CH}_{2} \mathrm{~F} \\
-178.963776 \\
-65.42\end{array}$ & 0.000441 & 0.276731 & -101.9 & \pm 1.3 \\
\hline \multicolumn{4}{|c|}{ Reported $\Delta_{\mathrm{f}} \mathrm{H}^{\circ}(298) \mathrm{kcal} \cdot \mathrm{mol}^{-1}$} & & & $-101.7 \pm 0.7$ & \\
\hline \multicolumn{4}{|c|}{ Standard Deviation over rxns } & & & \pm 0.1 & \\
\hline $\begin{array}{l}\mathrm{CH}_{3} \mathrm{CHFOH} \\
-254.19203\end{array}$ & $\begin{array}{l}+\mathrm{CH}_{3} \mathrm{CH}_{2} \mathrm{CH}_{3}= \\
-118.990915 \\
-25.02\end{array}$ & $\begin{array}{l}\mathrm{CH}_{3} \mathrm{CH}_{2} \mathrm{OH} \\
-154.926666 \\
-56.21\end{array}$ & $\begin{array}{l}+\mathrm{CH}_{3} \mathrm{CH}_{2} \mathrm{CH}_{2} \mathrm{~F} \\
-218.237381 \\
-70.24\end{array}$ & 0.018898 & 11.85867 & -113.3 & \pm 1.5 \\
\hline $\begin{array}{l}\mathrm{CH}_{3} \mathrm{CHFOH} \\
-254.19203\end{array}$ & $\begin{array}{l}+\mathrm{CH}_{3} \mathrm{CH}_{3}= \\
-79.717768 \\
-20.05\end{array}$ & $\begin{array}{l}\mathrm{CH}_{3} \mathrm{CH}_{2} \mathrm{OH} \\
-154.926666 \\
-56.21\end{array}$ & $\begin{array}{l}+\mathrm{CH}_{3} \mathrm{CH}_{2} \mathrm{~F} \\
-178.963776 \\
-65.42\end{array}$ & 0.019356 & 12.14606 & -113.7 & \pm 1.3 \\
\hline \multicolumn{4}{|c|}{ Reported $\Delta_{\mathrm{f}} \mathrm{H}^{\circ}(298) \mathrm{kcal} \cdot \mathrm{mol}^{-1}$} & & & $-113.5 \pm 1.4$ & \\
\hline \multicolumn{4}{|c|}{ Standard Deviation over rxns } & & & \pm 0.2 & \\
\hline $\begin{array}{l}\mathrm{C} \cdot \mathrm{HFCH}_{2} \mathrm{OH} \\
-253.518298\end{array}$ & $\begin{array}{l}+\mathrm{CH}_{3} \mathrm{CH}_{2} \mathrm{CH}_{3}= \\
-118.990915 \\
-25.02\end{array}$ & $\begin{array}{l}\mathrm{CH}_{3} \mathrm{CH}_{2} \mathrm{O} \bullet \\
-154.268107 \\
-3.01\end{array}$ & $\begin{array}{l}+\mathrm{CH}_{3} \mathrm{CH}_{2} \mathrm{CH}_{2} \mathrm{~F} \\
-218.237381 \\
-70.24\end{array}$ & 0.003725 & 2.337471 & -50.6 & \pm 1.4 \\
\hline $\begin{array}{l}\mathrm{C} \cdot \mathrm{HFCH}_{2} \mathrm{OH} \\
-253.518298\end{array}$ & $\begin{array}{l}+\mathrm{CH}_{4}= \\
-40.447961 \\
-17.81\end{array}$ & $\begin{array}{l}\mathrm{CH}_{3} \mathrm{CH}_{2} \mathrm{O} \bullet \\
-154.268107 \\
-3.01\end{array}$ & $\begin{array}{l}+\mathrm{CH}_{3} \mathrm{~F} \\
-139.683801 \\
-56.54\end{array}$ & 0.014351 & 9.005382 & -50.8 & \pm 0.1 \\
\hline \multicolumn{4}{|c|}{ Reported $\Delta_{\mathrm{f}} \mathrm{H}^{\circ}(298) \mathrm{kcal} \cdot \mathrm{mol}^{-1}$} & & & $-50.7 \pm 0.8$ & \\
\hline \multicolumn{4}{|c|}{ Standard Deviation over rxns } & & & \pm 0.1 & \\
\hline $\begin{array}{l}\mathrm{CH}_{2} \mathrm{FCH} \cdot \mathrm{OH} \\
-253.518298\end{array}$ & $\begin{array}{l}+\mathrm{CH}_{3} \mathrm{CH}_{3}= \\
-79.717768 \\
-20.05\end{array}$ & $\begin{array}{l}\mathrm{CH}_{3} \mathrm{CH}_{2} \mathrm{O} \bullet \\
-154.268107 \\
-3.01\end{array}$ & $\begin{array}{l}+\mathrm{CH}_{3} \mathrm{CH}_{2} \mathrm{~F} \\
-178.963776 \\
-65.42\end{array}$ & 0.012436 & 7.803702 & -56.2 & \pm 1.2 \\
\hline $\begin{array}{l}\mathrm{CH} 2 \mathrm{FCH} \bullet \mathrm{OH} \\
-253.518298\end{array}$ & $\begin{array}{l}+\mathrm{CH}_{4}= \\
-40.447961 \\
-17.81\end{array}$ & $\begin{array}{l}\mathrm{CH}_{3} \mathrm{CH}_{2} \mathrm{O} \bullet \\
-154.268107 \\
-3.01\end{array}$ & $\begin{array}{l}+\mathrm{CH}_{3} \mathrm{~F} \\
-139.683801 \\
-56.54\end{array}$ & 0.022604 & 14.18421 & -55.9 & \pm 0.1 \\
\hline Reported $\Delta_{\mathrm{f}} \mathrm{H}^{\circ}$ & 8) $\mathrm{kcal} \cdot \mathrm{mol}^{-1}$ & & & & & $-56.1 \pm 0.6$ & \\
\hline
\end{tabular}




\section{Continued}

Standard Deviation over rxns

$\begin{array}{llll}\mathrm{CH}_{2} \mathrm{FCH}_{2} \mathrm{O} \bullet & +\mathrm{CH}_{3} \mathrm{CH}_{2} \mathrm{CH}_{3}= & \mathrm{CH}_{3} \mathrm{CHO} \bullet & +\mathrm{CH}_{3} \mathrm{CH}_{2} \mathrm{CH}_{2} \mathrm{~F} \\ -253.509195 & -118.990915 & -154.268107 & -218.237381 \\ & -25.02 & -3.01 & -70.24 \\ & & & \\ \mathrm{CH}_{2} \mathrm{FCH}_{2} \mathrm{O} \bullet & +\mathrm{CH}_{4}= & \mathrm{CH}_{3} \mathrm{O} \cdot & +\mathrm{CH}_{3} \mathrm{CH}_{2} \mathrm{~F} \\ -253.509195 & -40.447961 & -114.989112 & -178.963776 \\ & -17.81 & 5.15 & -65.42\end{array}$

Reported $\Delta_{\mathrm{f}} \mathrm{H}^{\circ}(298) \mathrm{kcal} \cdot \mathrm{mol}^{-1}$

$$
-65.42
$$

Standard Deviation over rxns

$\begin{array}{llll}\mathrm{CH}_{2} \bullet \mathrm{CHFOH} & +\mathrm{CH}_{4}= & \mathrm{CH}_{3} \mathrm{O} \bullet & +\mathrm{CH}_{3} \mathrm{CH}_{2} \mathrm{~F} \\ -253.532134 & -40.447961 & -114.989112 & -178.963776 \\ & -17.81 & 5.15 & -65.42 \\ & & & \\ \mathrm{CH}_{2} \bullet \mathrm{CHFOH} & +\mathrm{CH}_{3} \mathrm{CH}_{3}= & \mathrm{CH}_{3} \mathrm{CH}_{2} \mathrm{O} \bullet & +\mathrm{CH}_{3} \mathrm{CH}_{2} \mathrm{~F} \\ -253.532134 & -79.717768 & -154.268107 & -178.963776 \\ & -20.05 & -3.01 & -65.42\end{array}$

Reported $\Delta_{\mathrm{f}} \mathrm{H}^{\circ}(298) \mathrm{kcal} \cdot \mathrm{mol}^{-1}$

Standard Deviation over rxns

$\begin{array}{llll}\mathrm{CH}_{3} \mathrm{CF} \cdot \mathrm{OH} & +\mathrm{CH}_{3} \mathrm{CH}_{3}= & \mathrm{CH}_{3} \mathrm{CH}_{2} \mathrm{O} \cdot & +\mathrm{CH}_{3} \mathrm{CH}_{2} \mathrm{~F} \\ -253.542527 & -79.717768 & -154.268107 & -178.963776 \\ & -20.05 & -5.01 & -65.42 \\ & & & \\ \mathrm{CH}_{3} \mathrm{CF} \cdot \mathrm{OH} & +\mathrm{CH}_{3} \mathrm{CH}_{2} \mathrm{CH}_{3}= & \mathrm{CH}_{3} \mathrm{CH}_{2} \mathrm{O} \cdot & +\mathrm{CH}_{3} \mathrm{CH}_{2} \mathrm{CH}_{2} \mathrm{~F} \\ -253.542527 & -118.990915 & -154.268107 & -218.237381 \\ & -25.02 & -5.01 & -70.24\end{array}$

Reported $\Delta_{\mathrm{f}} \mathrm{H}^{\circ}(298) \mathrm{kcal} \cdot \mathrm{mol}^{-1}$

Standard Deviation over rxns

$\begin{array}{llll}\mathrm{CH}_{3} \mathrm{CHFO} \bullet & +\mathrm{CH}_{4}= & \mathrm{CH}_{3} \mathrm{O} \bullet & +\mathrm{CH}_{3} \mathrm{CH}_{2} \mathrm{~F} \\ -253.530783 & -40.447961 & -114.989112 & -178.963776 \\ & -17.81 & 5.15 & -65.42 \\ \mathrm{CH}_{3} \mathrm{CHFO} \bullet & +\mathrm{CH}_{3} \mathrm{CH}_{3}= & \mathrm{CH}_{3} \mathrm{CH}_{2} \mathrm{O} \bullet & +\mathrm{CH}_{3} \mathrm{CH}_{2} \mathrm{~F} \\ -253.530783 & -79.717768 & -154.268107 & -178.963776 \\ & -20.05 & -3.01 & -65.42\end{array}$

Reported $\Delta_{\mathrm{f}} \mathrm{H}^{\circ}$ (298) kcal $\cdot \mathrm{mol}^{-1}$

Standard Deviation over rxns

$\begin{array}{llll}\mathrm{CH}_{2} \mathrm{FCHFOH} & +\mathrm{CH}_{4}= & \mathrm{CH}_{3} \mathrm{CH}_{2} \mathrm{OH} & +\mathrm{CH}_{2} \mathrm{~F}_{2} \\ -353.429924 & -40.447961 & -154.926666 & -238.938973 \\ & -17.81 & -56.21 & -108.07 \\ & & & \\ \mathrm{CH}_{2} \mathrm{FCHFOH} & +\mathrm{CH}_{3} \mathrm{CH}_{2} \mathrm{CH}_{3}= & \mathrm{CH}_{3} \mathrm{CH}_{2} \mathrm{OH} & +\mathrm{CH}_{3} \mathrm{CH}_{2} \mathrm{CHF}_{2} \\ -353.429924 & -118.990915 & -154.926666 & -317.498882 \\ & -25.02 & -56.21 & -125.82\end{array}$

Reported $\Delta_{\mathrm{f}} \mathrm{H}^{\circ}(298) \mathrm{kcal} \cdot \mathrm{mol}^{-1}$

Standard Deviation over rxns

\begin{tabular}{|c|c|c|c|c|c|c|c|}
\hline $\mathrm{CF}_{2} \mathrm{HCH}_{2} \mathrm{OH}$ & $+\mathrm{CH}_{4}=$ & $\mathrm{CH}_{3} \mathrm{CH}_{2} \mathrm{OH}$ & $+\mathrm{CH}_{2} \mathrm{~F}_{2}$ & & & & \\
\hline \multirow[t]{2}{*}{-353.431533} & 40.447961 & -154.926666 & -238.938973 & 0.013855 & 8.694137 & -155.2 & \pm 1.6 \\
\hline & -17.81 & -56.21 & -108.07 & & & & \\
\hline $\mathrm{CF}_{2} \mathrm{HCH}_{2} \mathrm{OH}$ & $+\mathrm{CH}_{3} \mathrm{CH}_{3}=$ & $\mathrm{CH}_{3} \mathrm{CH}_{2} \mathrm{OH}$ & $+\mathrm{CH}_{3} \mathrm{CHF}_{2}$ & & & & \\
\hline \multirow[t]{2}{*}{-353.431533} & -79.717768 & -154.926666 & -278.225303 & -0.00267 & -1.67419 & -155.4 & \pm 1.8 \\
\hline & -20.05 & -56.21 & -120.87 & & & & \\
\hline
\end{tabular}




\section{Continued}

Reported $\Delta_{\mathrm{f}} \mathrm{H}^{\circ}(298) \mathrm{kcal} \cdot \mathrm{mol}^{-1}$

Standard Deviation over rxns

$\begin{array}{llll}\mathrm{CH}_{3} \mathrm{CF}_{2} \mathrm{OH} & +\mathrm{CH}_{4}= & \mathrm{CH}_{3} \mathrm{CH}_{2} \mathrm{OH} & +\mathrm{CH}_{2} \mathrm{~F}_{2} \\ -353.462472 & -40.447961 & -154.926666 & -238.938973 \\ & -17.81 & -56.21 & -108.07 \\ & & & \\ \mathrm{CH}_{3} \mathrm{CF}_{2} \mathrm{OH} & +\mathrm{CH}_{3} \mathrm{CH}_{2} \mathrm{CH}_{3}= & \mathrm{CH}_{3} \mathrm{CH}_{2} \mathrm{OH} & +\mathrm{CH}_{3} \mathrm{CH}_{2} \mathrm{CHF}_{2} \\ -353.462472 & -118.990915 & -154.926666 & -317.498882 \\ & -25.02 & -56.21 & -125.82\end{array}$

Reported $\Delta_{\mathrm{f}} \mathrm{H}^{\circ}(298) \mathrm{kcal} \cdot \mathrm{mol}^{-1}$

Standard Deviation over rxns

$\begin{array}{llll}\mathrm{CH}_{2} \mathrm{FC} \cdot \mathrm{FOH} & +\mathrm{CH}_{4}= & \mathrm{CH}_{3} \mathrm{CH}_{2} \mathrm{O} \cdot & +\mathrm{CH}_{2} \mathrm{~F}_{2} \\ -352.776406 & -40.447961 & -154.268107 & -238.938973 \\ & -17.81 & -3.01 & -108.07 \\ & & & \\ \mathrm{CH}_{2} \mathrm{FC} \cdot \mathrm{FOH} & +\mathrm{CH}_{3} \mathrm{CH}_{2} \mathrm{CH}_{3}= & \mathrm{CH}_{3} \mathrm{CH}_{2} \mathrm{O} \cdot & +\mathrm{CH}_{3} \mathrm{CH}_{2} \mathrm{CHF}_{2} \\ -352.776406 & -118.990915 & -154.268107 & -317.498882 \\ & -25.02 & -3.01 & -125.82\end{array}$

Reported $\Delta_{\mathrm{f}} \mathrm{H}^{\circ}(298) \mathrm{kcal} \cdot \mathrm{mol}^{-1}$

Standard Deviation over rxns

$\begin{array}{llll}\mathrm{C} \cdot \mathrm{HFCFHOH} & +\mathrm{CH}_{4}= & \mathrm{CH}_{3} \mathrm{O} \cdot & +\mathrm{CH}_{3} \mathrm{CHF}_{2} \\ -352.778474 & -40.447961 & -114.989112 & -278.225303 \\ & -17.81 & 5.15 & -120.87 \\ \mathrm{C} \cdot \mathrm{HFCFHOH} & +\mathrm{CH}_{3} \mathrm{CH}_{3}= & \mathrm{CH}_{3} \mathrm{O} \cdot & +\mathrm{CH}_{3} \mathrm{CH}_{2} \mathrm{CHF}_{2} \\ -352.778474 & -79.717768 & -114.989112 & -317.498882 \\ & -20.05 & 5.15 & -125.82\end{array}$

Reported $\Delta_{\mathrm{f}} \mathrm{H}^{\circ}(298) \mathrm{kcal} \cdot \mathrm{mol}^{-1}$

Standard Deviation over rxns

$\begin{array}{ll}\mathrm{CHF}_{2} \mathrm{C} \cdot \mathrm{HOH} & +\mathrm{CH}_{4}= \\ -352.773245 & -40.447961 \\ & -17.81 \\ \mathrm{CHF}_{2} \mathrm{C} \cdot \mathrm{HOH} & +\mathrm{CH}_{3} \mathrm{CH}_{3}= \\ -352.773245 & -79.717768 \\ & -20.05\end{array}$

Reported $\Delta_{\mathrm{f}} \mathrm{H}^{\circ}(298) \mathrm{kcal} \cdot \mathrm{mol}^{-1}$

Standard Deviation over rxns

$\begin{array}{llll}\mathrm{CH}_{2} \mathrm{FCHFO} \bullet & +\mathrm{CH}_{4}= & \mathrm{CH}_{3} \mathrm{CH}_{2} \mathrm{O} \bullet & +\mathrm{CH}_{2} \mathrm{~F}_{2} \\ -352.768218 & -40.447961 & -154.268107 & -238.938973 \\ & -17.81 & -3.01 & -108.07 \\ & & \mathrm{CH}_{3} \mathrm{CH}_{2} \mathrm{O} \bullet & +\mathrm{CH}_{3} \mathrm{CHF}_{2} \\ \mathrm{CH}_{2} \mathrm{FCHFO} \bullet & +\mathrm{CH}_{3} \mathrm{CH}_{3}= & -154.268107 & -278.225303 \\ -352.768218 & -79.717768 & -3.01 & -120.87 \\ & -20.05 & & \end{array}$

Reported $\Delta_{\mathrm{f}} \mathrm{H}^{\circ}(298) \mathrm{kcal} \cdot \mathrm{mol}^{-1}$

Standard Deviation over rxns

$\begin{array}{ll}\mathrm{C} \cdot \mathrm{F}_{2} \mathrm{CH}_{2} \mathrm{OH} & +\mathrm{CH}_{4}= \\ -352.773245 & -40.447961 \\ & -17.81\end{array}$

$\begin{array}{ll}\mathrm{CH}_{3} \mathrm{O} \cdot & +\mathrm{CH}_{3} \mathrm{CHF}_{2} \\ -114.989112 & -278.225303 \\ 5.15 & -120.87 \\ & +\mathrm{CH}_{3} \mathrm{CH}_{2} \mathrm{CHF}_{2} \\ \mathrm{CH}_{3} \mathrm{O} \cdot & -317.498882 \\ -114.989112 & -125.82 \\ 5.15 & \end{array}$

$-155.3 \pm 1.7$

$\pm 0.1$

$\begin{array}{llll}0.044794 & 28.10864 & -174.6 & \pm 1.6 \\ & & & \\ 0.027839 & 17.46922 & -174.5 & \pm 1.5\end{array}$

$-174.5 \pm 1.5$

$\pm 0.1$

0.01728

10.84775

$-104.1$

$\pm 1.5$

0.000332

0.208333

$-104.0$

$\pm 1.4$

$-104.1 \pm 1.5$

$\pm 0.1$

$\begin{array}{llll}0.01202 & 7.542658 & -105.5 & \pm 1.7\end{array}$

$\begin{array}{llll}0.008248 & 5.175694 & -105.8 & \pm 1.8\end{array}$

$-105.6 \pm 1.7$

$\pm 0.2$

$\begin{array}{llll}0.006791 & 4.261414 & -102.5 & \pm 1.7\end{array}$

$\begin{array}{llll}0.003019 & 1.89445 & -102.2 & \pm 1.8\end{array}$

$-102.3 \pm 1.7$

$\pm 0.2$

$\begin{array}{llll}0.009099 & 5.709704 & -99.0 & \pm 1.5\end{array}$

$\begin{array}{llll}-0.00742 & -4.65863 & -99.2 & \pm 1.7\end{array}$

$-99.1 \pm 1.6$

$\pm 0.1$

$\begin{array}{llll}0.014126 & 8.864192 & -102.1 & \pm 1.5\end{array}$ 
Continued

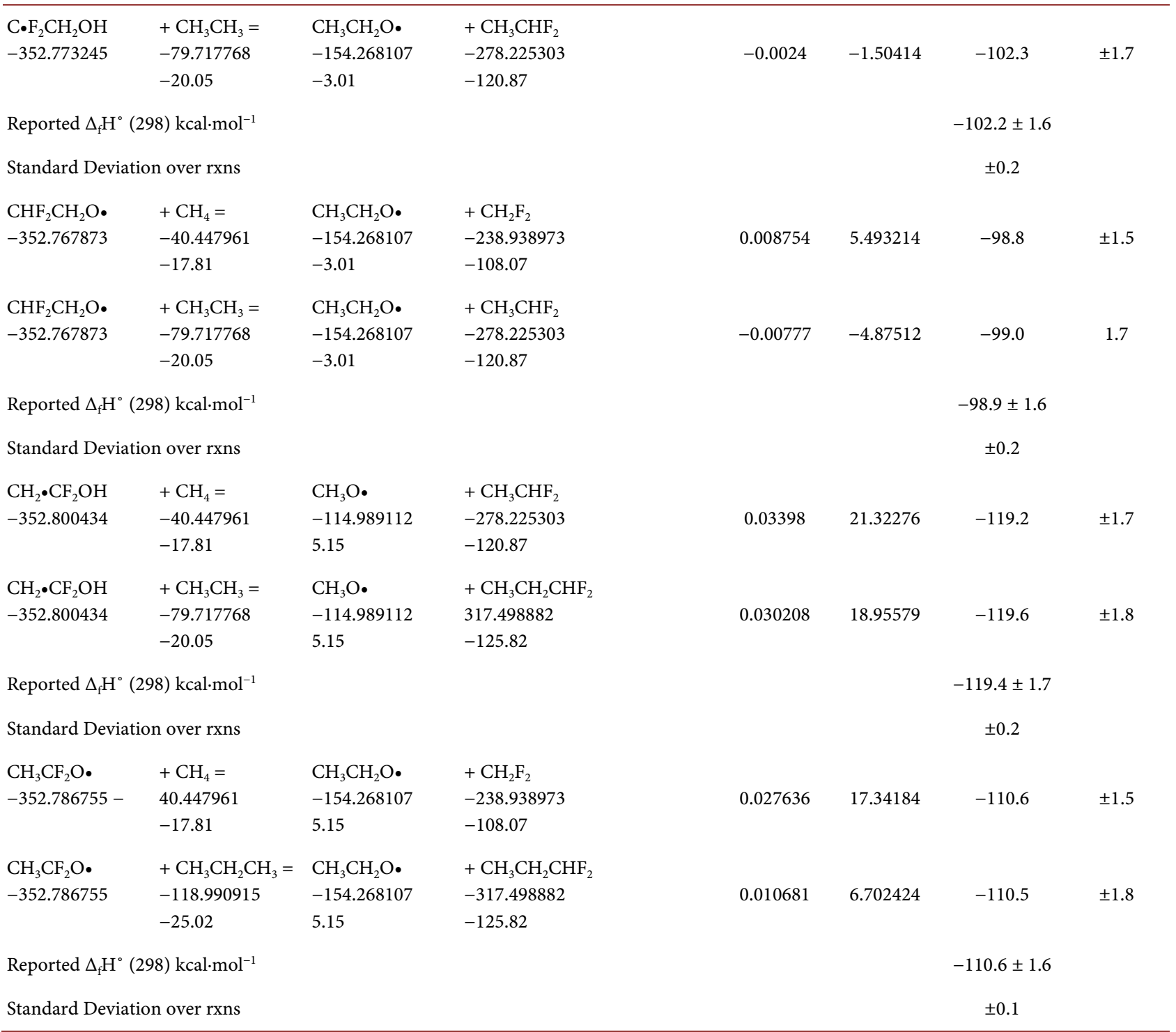

Hartrees, $\mathrm{kcal} \cdot \mathrm{mole}^{-1}{ }^{*} \mathrm{SD}$ Standard Deviation $\mathrm{kcal} \cdot \mathrm{mol}^{-1}$ Errors reported avg of sum of uncertainties in rxn's reference species.

standard deviation [19] and example calculation(s) are shown in the Supplemental Information table provided. The bond dissociation energy (BDE's) of Monofluoro and Difluoro-Ethanol's are also calculated in Table 5 with a small percent error, ranging from $0.2-0.8 \mathrm{Kcal} / \mathrm{mol}$. A small value for percent error indicates accurate calculated values for $\mathrm{BDB}$ of these molecules.

\subsection{Bond Energies}

\section{*Supplemental Information}

Supplemental Information is available, Cartesian Coordinates; Z-matrixes, vibration frequencies, moments of inertia, the method of standard deviation, Optimized Geometries, and C-C and C-O internal rotors potential energy profile for target fluorinated ethanol and their related radicals are included. 
Table 5. Bond Dissociation Energy (BDE's) of Monofluoro and Difluoro-Ethanol's.

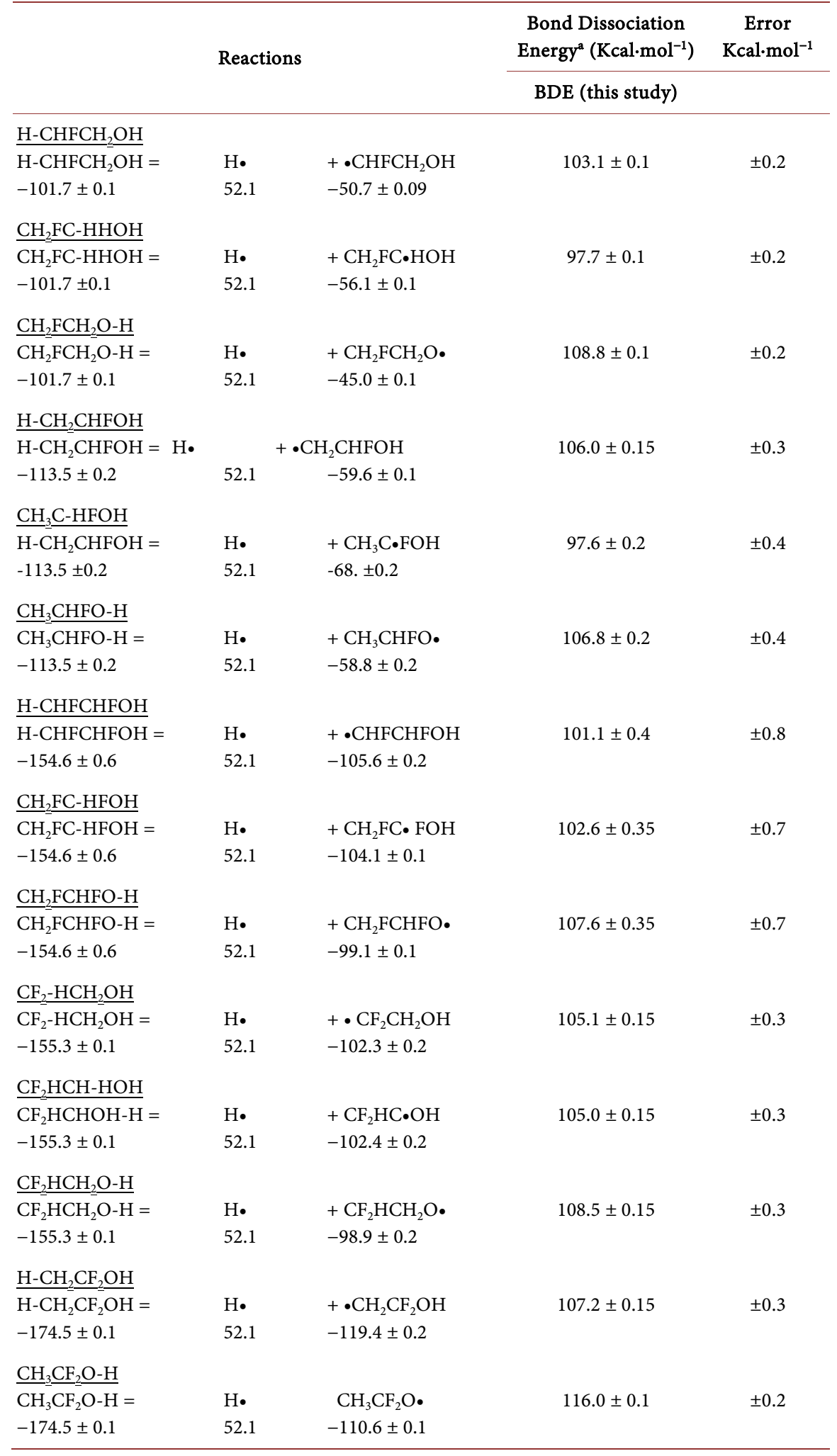

\section{Conclusions}

Thermodynamic properties of 19 mono and di-fluoro ethanols and their related 
radicals are calculated using the $a b$ initio and Global-hybrid meta-GGA density function methods. Isodesmic work reactions are employed for cancellation of calculation errors. Multiple work reactions are utilized to calculate standard enthalpy of formation at the Gaussian M06-2X calculation level. Optimized geometries and frequencies are used to determine entropy and heat capacity with M06-2x/6-31 + g (d, p) level of calculation. Intermolecular torsion potential curves at the M-06-2x/6-31 $+\mathrm{g}(\mathrm{d}, \mathrm{p})$ level of calculation are used to calculate hindered internal rotation contributions to heat capacity and entropy with a correction to the calculated heat capacity and entropy. The Thermochemical properties: Entropy, Heat Capacities at $\left(298^{\circ}-1500^{\circ} \mathrm{K}\right)$, Standard Enthalpy of formation (298K), and the $\mathrm{C}-\mathrm{F}$ and $\mathrm{C}-\mathrm{H}$ Bond Dissociation Energies (BDEs) for Mono and Difluorinated Ethanols and Radicals: $\mathrm{CH}_{3-\mathrm{x}} \mathrm{CHF}_{\mathrm{x}} \mathrm{OH}, \mathrm{CH}_{3} \mathrm{CH}_{2-\mathrm{x}} \mathrm{F}_{\mathrm{x}} \mathrm{OH}$ have been calculated. The $\mathrm{C}-\mathrm{H}$ bond energies range from 102.2 to $107.2 \mathrm{Kcal} \cdot \mathrm{mol}^{-1}$ on the methyl carbons, and from 97.3 to $105.2 \mathrm{Kcal} \cdot \mathrm{mol}^{-1}$ on the secondary ethyl carbons. The calculated values for $\mathrm{C}-\mathrm{H}$ bond energies for fluorinated methyl carbons are higher than those of the fluorinated ethyl carbons. Calculated values for the $\mathrm{O}-\mathrm{H}$ bond energies for 2-fluoroethanol are higher than those of $\mathrm{O}-\mathrm{H}$ bond energies for 1-fluoroethanol and an intermediate calculated value for $\mathrm{O}-\mathrm{H}$ bond energies for 1,2-difluoroethanol. Introducing a fluorine atom to either methyl or ethyl carbon increases O-H bond energy [18].

\section{Acknowledgements}

We acknowledge the NJIT Advanced Research Computing Services for significant help in providing the computer calculation software.

\section{Conflicts of Interest}

The authors declare no conflicts of interest regarding the publication of this paper.

\section{References}

[1] Wallington, T.J., Schneider, W.F., Worsnop, D.R., Nielsen, O.J., Sehested, J., Debruyn, W.J. and Shorter, J.A. (1994) The Environmental Impact of CFC Replacements HFCs and HCFCs. Environmental Science \& Technology, 28, 320A-326A. https://doi.org/10.1021/es00056a714

[2] Wang, H., Castillo, A. and Bozzelli, J.W. (2015) Thermochemical Properties Enthalpy, Entropy, and Heat Capacity of C1-C4 Fluorinated Hydrocarbons: Fluorocarbon Group Additivity. The Journal of Physical Chemistry A, 119, 8202-8215. https://doi.org/10.1021/acs.jpca.5b03912

[3] Schneider, W.F. and Wallington, T.J. (1993) Ab Initio Investigation of the Heats of Formation of Several Trifluoromethyl Compounds. The Journal of Physical Chemistry, 97, 12783-12788. https://doi.org/10.1021/j100151a025

[4] Wang, H. and Bozzelli, J.W. (2016) Thermochemical Properties and Bond Dissociation Energies for Fluorinated Methanol, $\mathrm{CH}_{3-x} \mathrm{~F}_{x} \mathrm{OH}$, and Fluorinated Methyl Hydroperoxides, $\mathrm{CH}_{3-x} \mathrm{~F}_{x} \mathrm{OOH}$ : Group Additivity. The Journal of Physical Chemistry 
$A, 120,6998-7010$.

[5] Ruscic, B. (2015) Active Thermochemical Tables: Sequential Bond Dissociation Enthalpy of Methane, Ethane, and Methanol and Related Thermochemistry. The Journal of Physical Chemistry A, 119, 7810-7837. https://doi.org/10.1021/acs.jpca.5b01346

[6] Burke, S.M., Simmie, J.M. and Curran, H.J. (2015) Critical Evaluation of Thermochemical Properties of C1-C4 Species: Updated Group Contributions to Estimate Thermochemical Properties. Journal of Physical and Chemical Reference Data, 44, Article ID: 013101. https://doi.org/10.1063/1.4902535

[7] Chase, M.W.J. (1998) NIST-JANAF Thermochemical Tables (Journal of Physical and Chemical Reference Data). Monograph No. 9, 1-1951.

[8] Luo, X., Fleming, P.R. and Rizzo, T.R. (1992) Vibrational Overtone Spectroscopy of the $4 \varkappa \mathrm{OH}+\mathrm{OH}^{\prime}$ Combination Level of $\mathrm{HOOH}$ via Sequential Local Mode-Local Mode Excitation. The Journal of Chemical Physics, 96, 5659-5667. https://doi.org/10.1063/1.462665

[9] Bodi, A., Kercher, J.P., Bond, C., Meteesatien, P., Sztaray, B. and Baer, T. (2006) Photoion Photoelectron Coincidence Spectroscopy of Primary Amines $\mathrm{RCH}_{2} \mathrm{NH}_{2}$ $\left(\mathrm{R}=\mathrm{H}, \mathrm{CH}_{3}, \mathrm{C}_{2} \mathrm{H}_{5}, \mathrm{C}_{3} \mathrm{H}_{7}, \mathrm{i}-\mathrm{C}_{3} \mathrm{H}_{7}\right)$ : Alkylamine and Alkyl Radical Heats of Formation by Isodesmic Reaction Networks. The Journal of Physical Chemistry A, 110, 13425-13433. https://doi.org/10.1021/jp064739s

[10] Wang, H. and Bozzelli, J.W. (2016) Thermochemical Properties $\left(\Delta \mathrm{fH}^{\circ}(298 \mathrm{~K})\right.$, $\left.\mathrm{S}^{\circ}(298 \mathrm{~K}), \mathrm{C} \mathrm{p}(\mathrm{T})\right)$ and Bond Dissociation Energies for C1-C4 Normal Hydroperoxides and Peroxy Radicals. Journal of Chemical \& Engineering Data, 61, 1836-1849. https://doi.org/10.1021/acs.jced.5b01035

[11] Csontos, J., Rolik, Z., Das, S. and Kallay, M. (2010) High-Accuracy Thermochemistry of Atmospherically Important Fluorinated and Chlorinated Methane Derivatives. The Journal of Physical Chemistry A, 114, 13093-13103. https://doi.org/10.1021/jp105268m

[12] Expanding the Limits of Computational Chemistry, 2015-2019 (2020) Density Function Method. https://gaussian.com/dft

[13] NIST Computational Chemistry Comparison and Benchmark Database, NIST Standard Reference Database Number 101, Release 16a; Johnson, R.D. III, Ed., NIST: Gaithersburg, MD. http://cccbdb.nist.gov

[14] Sheng, C. (2002) Elementary, Pressure Dependent Model for Combustion of $C_{1}, C_{2}$ and Nitrogen Containing Hydrocarbons: Operation of a Pilot Scale Incinerator and Model Comparison. Ph.D. Dissertation, New Jersey Institute of Technology, Newark.

[15] Becke, A.D. (1993) Density-Functional Thermochemistry. III. The Role of Exact Exchange. The Journal of Chemical Physics, 98, 5648-5652. https://doi.org/10.1063/1.464913

[16] Lay, T.H., Krasnoperov, L.N., Venanzi, C.A., Bozzelli, J.W. and Shokhirev, N.V. (1996) $A b$ Initio Study of $\alpha$-Chlorinated Ethyl Hydro Peroxides $\mathrm{CH}_{3} \mathrm{CH}_{2} \mathrm{OOH}$, $\mathrm{CH}_{3} \mathrm{CHClOOH}$, and $\mathrm{CH}_{3} \mathrm{CCl}_{2} \mathrm{OOH}$ : Conformational Analysis, Internal Rotation Barriers, Vibrational Frequencies, and Thermodynamic Properties. The Journal of Physical Chemistry, 100, 8240-8249. https://doi.org/10.1021/jp952976h

[17] Math Is Fun Advanced, 2017. Standard Deviation and Variance. https://www.mathsisfun.com/data/standard-deviation.html

[18] Myrna, H.M., Minh, T.N., David, A.D. and Minh, T.N. (2007) Theoretical Predic- 
tion of the Heats of Formation of $\mathrm{C}_{2} \mathrm{H}_{5} \mathrm{O}$ Radicals Derived from Ethanol and of the Kinetics of Beta-C-C Scission in the Ethoxy Radical. The Journal of Physical Chemistry $A, 111,113-126$. 


\section{Supplemental Information}

Table S1. Cartesian Coordinates in Angstroms for target fluorinated ethanol and their related radical's geometries at the $\mathrm{m} 062 \mathrm{x} / 6-31+\mathrm{g}(\mathrm{d}, \mathrm{p})$ level of theory.

\begin{tabular}{|c|c|c|c|c|}
\hline \multicolumn{2}{|c|}{$\mathrm{CH}_{2} \mathrm{FCH}_{2} \mathrm{OH}$} & \multicolumn{3}{|c|}{ Cartesian Coordinates } \\
\hline Center Number & Atomic Number & $\mathrm{X}$ & $\mathrm{Y}$ & $\mathrm{Z}$ \\
\hline 1 & 6 & 0.000003116 & -0.000001518 & 0.000020526 \\
\hline 2 & 9 & 0.000014524 & -0.000002341 & -0.000029060 \\
\hline 3 & 1 & -0.000005137 & -0.000003977 & 0.000002345 \\
\hline 4 & 1 & 0.000001564 & 0.000000767 & 0.000016765 \\
\hline 5 & 6 & -0.000021746 & 0.000030833 & 0.000003239 \\
\hline 6 & 1 & -0.000002396 & 0.000010185 & 0.000006184 \\
\hline 7 & 1 & 0.000005782 & -0.000006013 & -0.000000243 \\
\hline 8 & 8 & 0.000005060 & -0.000028332 & -0.000029361 \\
\hline 9 & 1 & -0.000000767 & 0.000000396 & 0.000009605 \\
\hline \multicolumn{2}{|c|}{$\mathrm{CH}_{3} \mathrm{CHFOH}$} & \multicolumn{3}{|c|}{ Cartesian Coordinates } \\
\hline Center Number & Atomic Number & $\mathrm{X}$ & $\mathrm{Y}$ & $\mathrm{Z}$ \\
\hline 1 & 6 & 0.000021353 & 0.000006161 & -0.000053662 \\
\hline 2 & 6 & -0.000117652 & 0.000027420 & 0.000072872 \\
\hline 3 & 1 & -0.000017542 & -0.000011156 & -0.000000420 \\
\hline 4 & 1 & 0.000002761 & -0.000007600 & 0.000015768 \\
\hline 5 & 1 & -0.000013646 & -0.000002428 & 0.000033080 \\
\hline 6 & 9 & 0.000109835 & 0.000008063 & -0.000042913 \\
\hline 7 & 1 & 0.000018820 & -0.000022248 & 0.000004569 \\
\hline 8 & 8 & 0.000000477 & 0.000011019 & -0.000059799 \\
\hline 9 & 1 & -0.000004406 & -0.000009231 & 0.000030505 \\
\hline \multicolumn{2}{|c|}{$\mathrm{C} \cdot \mathrm{HFCH}_{2} \mathrm{OH}$} & \multicolumn{3}{|c|}{ Cartesian Coordinates } \\
\hline Center Number & Atomic Number & $\mathrm{X}$ & $\mathrm{Y}$ & $\mathrm{Z}$ \\
\hline 1 & 6 & -0.000020602 & 0.000003182 & -0.000077408 \\
\hline 2 & 9 & 0.000008151 & 0.000053880 & 0.000000565 \\
\hline 3 & 1 & -0.000000850 & -0.000011335 & 0.000020759 \\
\hline 4 & 6 & 0.000027899 & -0.000023254 & 0.000011757 \\
\hline 5 & 1 & -0.000003824 & -0.000056264 & -0.000008549 \\
\hline 6 & 1 & 0.000008293 & 0.000001847 & -0.000024269 \\
\hline 7 & 8 & -0.000008585 & -0.000003122 & 0.000024031 \\
\hline 8 & 1 & -0.000010483 & 0.000035066 & 0.000053114 \\
\hline \multicolumn{2}{|c|}{$\mathrm{CH}_{2} \mathrm{FCH} \cdot \mathrm{OH}$} & \multicolumn{3}{|c|}{ Cartesian Coordinates } \\
\hline Center Number & Atomic Number & $\mathrm{X}$ & $\mathrm{Y}$ & $\mathrm{Z}$ \\
\hline 1 & 6 & 0.000001249 & -0.000000923 & -0.000001559 \\
\hline 2 & 9 & 0.000007760 & 0.000016425 & -0.000015375 \\
\hline 3 & 1 & -0.000003551 & 0.000003238 & -0.000020167 \\
\hline 4 & 1 & -0.000005664 & 0.000000417 & -0.000013020 \\
\hline 5 & 6 & -0.000037395 & 0.000046885 & 0.000022542 \\
\hline 6 & 1 & 0.000001277 & -0.000023053 & 0.000004842 \\
\hline 7 & 8 & 0.000037801 & -0.000035478 & -0.000015526 \\
\hline 8 & 1 & -0.000001476 & -0.000007512 & 0.000038263 \\
\hline
\end{tabular}




\section{Continued}

\begin{tabular}{|c|c|c|c|c|}
\hline \multicolumn{2}{|c|}{$\mathrm{CH}_{2} \mathrm{FCH}_{2} \mathrm{O} \bullet$} & \multicolumn{3}{|c|}{ Cartesian Coordinates } \\
\hline Center Number & Atomic Number & $\mathrm{X}$ & $\mathrm{Y}$ & $\mathrm{Z}$ \\
\hline 1 & 6 & 0.000009752 & 0.000030682 & 0.000120978 \\
\hline 2 & 9 & -0.000007540 & 0.000018428 & -0.000104164 \\
\hline 3 & 1 & -0.000006175 & -0.000009977 & -0.000011573 \\
\hline 4 & 1 & -0.000001921 & -0.000005002 & -0.000038255 \\
\hline 5 & 6 & -0.000095473 & -0.000015917 & 0.000025447 \\
\hline 6 & 1 & 0.000036312 & -0.000025722 & 0.000008467 \\
\hline 7 & 1 & 0.000030627 & 0.000027608 & -0.000011892 \\
\hline 8 & 8 & 0.000034418 & -0.000020100 & 0.000010992 \\
\hline \multicolumn{2}{|c|}{$\mathrm{CH}_{2} \bullet \mathrm{CHFOH}$} & \multicolumn{3}{|c|}{ Cartesian Coordinates } \\
\hline Center Number & Atomic Number & $\mathrm{X}$ & $\mathrm{Y}$ & Z \\
\hline 1 & 6 & 0.000014779 & 0.000033223 & -0.000005569 \\
\hline 2 & 6 & -0.000030495 & -0.000005099 & 0.000010101 \\
\hline 3 & 1 & 0.000010354 & -0.000009796 & -0.000011520 \\
\hline 4 & 1 & -0.000018812 & -0.000018702 & -0.000000754 \\
\hline 5 & 9 & 0.000041121 & 0.000009214 & -0.000011106 \\
\hline 6 & 1 & 0.000011136 & -0.000017220 & 0.000008038 \\
\hline 7 & 8 & -0.000010627 & 0.000005847 & 0.000010223 \\
\hline 8 & 1 & -0.000017456 & -0.000007665 & 0.000000588 \\
\hline \multicolumn{2}{|c|}{$\mathrm{CH}_{3} \mathrm{CF} \cdot \mathrm{OH}$} & \multicolumn{3}{|c|}{ Cartesian Coordinates } \\
\hline Center Number & Atomic Number & $\mathrm{X}$ & $\mathrm{Y}$ & $\mathrm{Z}$ \\
\hline 1 & 6 & 0.000001336 & 0.000012708 & -0.000046895 \\
\hline 2 & 6 & -0.000071306 & 0.000036407 & 0.000029101 \\
\hline 3 & 1 & -0.000014952 & -0.000021374 & -0.000009157 \\
\hline 4 & 1 & -0.000000203 & 0.000002487 & 0.000002208 \\
\hline 5 & 1 & -0.000029193 & 0.000004057 & 0.000022548 \\
\hline 6 & 9 & 0.000068515 & 0.000006342 & -0.000002103 \\
\hline 7 & 8 & 0.000028358 & -0.000032227 & 0.000010729 \\
\hline 8 & 1 & 0.000017444 & -0.000008399 & -0.000006430 \\
\hline \multicolumn{2}{|c|}{$\mathrm{CH}_{3} \mathrm{CHFO} \bullet$} & \multicolumn{3}{|c|}{ Cartesian Coordinates } \\
\hline Center Number & Atomic Number & $\mathrm{X}$ & $\mathrm{Y}$ & $\mathrm{Z}$ \\
\hline 1 & 6 & -0.000025583 & 0.000026726 & 0.000006931 \\
\hline 2 & 6 & -0.000004925 & -0.000017774 & -0.000022873 \\
\hline 3 & 1 & -0.000016576 & 0.000000727 & -0.000013731 \\
\hline 4 & 1 & -0.000010431 & 0.000004336 & 0.000014687 \\
\hline 5 & 1 & -0.000006608 & 0.000007692 & -0.000003229 \\
\hline 6 & 9 & 0.000038154 & -0.000008832 & 0.000013405 \\
\hline 7 & 1 & 0.000017550 & -0.000008997 & -0.000000815 \\
\hline 8 & 8 & 0.000008419 & -0.000003878 & 0.000005626 \\
\hline
\end{tabular}




\section{Continued}

\begin{tabular}{|c|c|c|c|c|}
\hline \multicolumn{2}{|c|}{$\mathrm{CH}_{2} \mathrm{FCHFOH}$} & \multicolumn{3}{|c|}{ Cartesian Coordinates } \\
\hline Center Number & Atomic Number & $\mathrm{X}$ & Y & Z \\
\hline 1 & 6 & -0.000209387 & -0.000014742 & -0.000046791 \\
\hline 2 & 6 & -0.000078791 & -0.000026117 & -0.000054220 \\
\hline 3 & 9 & 0.000055970 & -0.000014001 & 0.000038779 \\
\hline 4 & 1 & 0.000037582 & 0.000003431 & 0.000025080 \\
\hline 5 & 1 & 0.000008069 & -0.000003401 & 0.000000786 \\
\hline 6 & 9 & 0.000213499 & -0.000011196 & 0.000078222 \\
\hline 7 & 1 & -0.000002897 & 0.000018025 & -0.000024730 \\
\hline 8 & 8 & -0.000003076 & 0.000003947 & -0.000028849 \\
\hline 9 & 1 & -0.000020969 & 0.000044055 & 0.000011724 \\
\hline \multicolumn{2}{|c|}{$\mathrm{CF}_{2} \mathrm{HCH}_{2} \mathrm{OH}$} & \multicolumn{3}{|c|}{ Cartesian Coordinates } \\
\hline Center Number & Atomic Number & $\mathrm{X}$ & Y & Z \\
\hline 1 & 6 & -0.000006264 & -0.000010001 & -0.000015081 \\
\hline 2 & 6 & 0.000196771 & -0.000004891 & 0.000062245 \\
\hline 3 & 9 & -0.000177152 & -0.000024722 & -0.000031629 \\
\hline 4 & 9 & 0.000058924 & 0.000072953 & -0.000005858 \\
\hline 5 & 1 & -0.000009820 & 0.000002957 & -0.000026980 \\
\hline 6 & 1 & -0.000016810 & -0.000024610 & 0.000029199 \\
\hline 7 & 1 & -0.000014380 & 0.000016248 & -0.000024677 \\
\hline 8 & 8 & -0.000035113 & -0.000034435 & 0.000010637 \\
\hline 9 & 1 & 0.000003845 & 0.000006502 & 0.000002145 \\
\hline \multicolumn{2}{|c|}{$\mathrm{CH}_{3} \mathrm{CF}_{2} \mathrm{OH}$} & \multicolumn{3}{|c|}{ Cartesian Coordinates } \\
\hline Center Number & Atomic Number & $\mathrm{X}$ & $\mathrm{Y}$ & $\mathrm{Z}$ \\
\hline 1 & 6 & 0.000056837 & -0.000094451 & 0.000110833 \\
\hline 2 & 6 & -0.000057651 & 0.000098699 & 0.000001478 \\
\hline 3 & 1 & 0.000011317 & -0.000004093 & -0.000026345 \\
\hline 4 & 1 & 0.000014600 & -0.000025891 & -0.000015228 \\
\hline 5 & 1 & -0.000001272 & -0.000012112 & -0.000025019 \\
\hline 6 & 9 & -0.000131047 & -0.000088396 & 0.000020206 \\
\hline 7 & 9 & 0.000140461 & 0.000063879 & 0.000020905 \\
\hline 8 & 8 & -0.000014132 & 0.000020314 & -0.000026071 \\
\hline 9 & 1 & -0.000019113 & 0.000042051 & -0.000060759 \\
\hline \multicolumn{2}{|c|}{$\mathrm{CH}_{2} \mathrm{FC} \cdot \mathrm{FOH}$} & \multicolumn{3}{|c|}{ Cartesian Coordinates } \\
\hline Center Number & Atomic Number & $\mathrm{X}$ & $\mathrm{Y}$ & Z \\
\hline 2 & 6 & 0.000107121 & 0.000054590 & 0.000053325 \\
\hline 3 & 9 & -0.000086887 & -0.000026092 & -0.000005448 \\
\hline 4 & 1 & -0.000015313 & -0.000013867 & -0.000003160 \\
\hline 5 & 1 & 0.000000240 & -0.000001842 & -0.000016239 \\
\hline 6 & 9 & 0.000003934 & 0.000030284 & 0.000010058 \\
\hline 7 & 8 & 0.000076030 & 0.000051318 & -0.000056283 \\
\hline 8 & 1 & 0.000000747 & 0.000022936 & 0.000051063 \\
\hline
\end{tabular}




\section{Continued}

\begin{tabular}{|c|c|c|c|c|}
\hline \multicolumn{2}{|c|}{$\mathrm{C} \cdot \mathrm{HFCFHOH}$} & \multicolumn{3}{|c|}{ Cartesian Coordinates } \\
\hline Center Number & Atomic Number & $\mathrm{x}$ & $\mathrm{Y}$ & $\mathrm{Z}$ \\
\hline 1 & 6 & -0.000058756 & 0.000023216 & 0.000035904 \\
\hline 2 & 6 & -0.000088748 & -0.000108788 & -0.000099552 \\
\hline 3 & 9 & 0.000068251 & 0.000068970 & 0.000065027 \\
\hline 4 & 1 & 0.000023870 & 0.000025841 & 0.000034527 \\
\hline 5 & 9 & 0.000011825 & -0.000021999 & -0.000019250 \\
\hline 6 & 1 & -0.000011611 & 0.000023111 & -0.000006306 \\
\hline 7 & 8 & 0.000047202 & 0.000013830 & -0.000008891 \\
\hline 8 & 1 & 0.000007966 & -0.000024181 & -0.000001459 \\
\hline \multicolumn{2}{|c|}{$\mathrm{CH}_{2} \mathrm{FCHFO} \bullet$} & \multicolumn{3}{|c|}{ Cartesian Coordinates } \\
\hline Center Number & Atomic Number & $\mathrm{X}$ & $\mathrm{Y}$ & $\mathrm{Z}$ \\
\hline 1 & 6 & -0.000043639 & 0.000033246 & 0.000009821 \\
\hline 2 & 6 & 0.000048716 & -0.000026830 & -0.000018036 \\
\hline 3 & 9 & -0.000022444 & 0.000006378 & -0.000008784 \\
\hline 4 & 1 & 0.000001695 & 0.000020117 & 0.000006913 \\
\hline 5 & 1 & 0.000003424 & 0.000009684 & 0.000001110 \\
\hline 6 & 9 & 0.000023881 & 0.000019171 & 0.000030292 \\
\hline 7 & 1 & 0.000006948 & -0.000005119 & 0.000004871 \\
\hline 8 & 8 & -0.000018580 & -0.000056646 & -0.000026187 \\
\hline \multicolumn{2}{|c|}{$\mathrm{CHF}_{2} \mathrm{C} \cdot \mathrm{HOH}$} & \multicolumn{3}{|c|}{ Cartesian Coordinates } \\
\hline Center Number & Atomic Number & $\mathrm{x}$ & $\mathrm{Y}$ & $\mathrm{Z}$ \\
\hline 1 & 6 & 0.000004676 & -0.000070251 & 0.000051141 \\
\hline 2 & 6 & 0.000027275 & -0.000132737 & -0.000118944 \\
\hline 3 & 1 & -0.000013476 & 0.000008382 & 0.000015950 \\
\hline 4 & 8 & 0.000006557 & 0.000005715 & -0.000023257 \\
\hline 5 & 1 & 0.000018970 & -0.000003557 & 0.000007094 \\
\hline 6 & 1 & -0.000051957 & -0.000006265 & -0.000015262 \\
\hline 7 & 9 & -0.000013983 & 0.000033076 & 0.000051050 \\
\hline 8 & 9 & 0.000021937 & 0.000165638 & 0.000032227 \\
\hline \multicolumn{2}{|c|}{$\mathrm{CF}_{2} \cdot \mathrm{CFOH}$} & \multicolumn{3}{|c|}{ Cartesian Coordinates } \\
\hline Center Number & Atomic Number & $\mathrm{x}$ & $\mathrm{Y}$ & $\mathrm{Z}$ \\
\hline 1 & 6 & 0.000045030 & 0.000052085 & -0.000051151 \\
\hline 2 & 6 & 0.000001125 & 0.000036125 & 0.000024114 \\
\hline 3 & 1 & -0.000002716 & 0.000001155 & 0.000006275 \\
\hline 4 & 8 & -0.000050429 & -0.000036049 & 0.000025853 \\
\hline 5 & 1 & -0.000005831 & -0.000009141 & -0.000009552 \\
\hline 6 & 1 & -0.000015223 & 0.000001185 & 0.000012909 \\
\hline 7 & 9 & 0.000011981 & -0.000006184 & 0.000002242 \\
\hline 8 & 9 & 0.000016063 & -0.000039174 & -0.000010691 \\
\hline
\end{tabular}




\section{Continued}

\begin{tabular}{|c|c|c|c|c|}
\hline \multicolumn{2}{|c|}{$\mathrm{CHF}_{2} \mathrm{CH}_{2} \mathrm{O} \bullet$} & \multicolumn{3}{|c|}{ Cartesian Coordinates } \\
\hline Center Number & Atomic Number & $\mathrm{X}$ & $\mathrm{Y}$ & $\mathrm{Z}$ \\
\hline 1 & 6 & -0.000032920 & -0.000119205 & 0.000076072 \\
\hline 2 & 6 & -0.000030593 & -0.000022551 & -0.000053428 \\
\hline 3 & 9 & -0.000036099 & 0.000010735 & 0.000025263 \\
\hline 4 & 9 & -0.000006345 & -0.000017725 & -0.000018129 \\
\hline 5 & 1 & -0.000014790 & -0.000026477 & 0.000021461 \\
\hline 6 & 1 & 0.000040237 & 0.000019571 & -0.000021746 \\
\hline 7 & 1 & 0.000038791 & 0.000046905 & -0.000010985 \\
\hline 8 & 8 & 0.000041718 & 0.000108747 & -0.000018508 \\
\hline \multicolumn{2}{|c|}{$\mathrm{CH}_{2} \cdot \mathrm{CF}_{2} \mathrm{OH}$} & \multicolumn{3}{|c|}{ Cartesian Coordinates } \\
\hline Center Number & Atomic Number & $\mathrm{X}$ & $\mathrm{Y}$ & $\mathrm{Z}$ \\
\hline 2 & 6 & -0.000001071 & -0.000001757 & -0.000000838 \\
\hline 3 & 1 & 0.000004590 & 0.000002074 & 0.000000801 \\
\hline 4 & 1 & 0.000019461 & 0.000007677 & -0.000000682 \\
\hline 5 & 9 & -0.000009537 & -0.000013694 & -0.000009305 \\
\hline 6 & 9 & -0.000017948 & 0.000004296 & 0.000010263 \\
\hline 7 & 8 & 0.000001500 & 0.000001265 & 0.000000690 \\
\hline 8 & 1 & -0.000006214 & -0.000001754 & -0.000000488 \\
\hline \multicolumn{2}{|c|}{$\mathrm{CH}_{3} \mathrm{CF}_{2} \mathrm{O} \bullet$} & \multicolumn{3}{|c|}{ Cartesian Coordinates } \\
\hline Center Number & Atomic Number & $\mathrm{X}$ & $\mathrm{Y}$ & $\mathrm{Z}$ \\
\hline 1 & 6 & -0.000049808 & 0.000049564 & 0.000101113 \\
\hline 2 & 6 & 0.000314370 & -0.000446227 & -0.000322008 \\
\hline 3 & 1 & -0.000001882 & 0.000003042 & 0.000019595 \\
\hline 4 & 1 & 0.000007442 & 0.000003265 & -0.000034062 \\
\hline 5 & 1 & 0.000007479 & -0.000020681 & 0.000033341 \\
\hline 6 & 9 & -0.000225548 & 0.000114125 & 0.000063416 \\
\hline 7 & 9 & -0.000008257 & 0.000232988 & 0.000078418 \\
\hline 8 & 8 & -0.000043796 & 0.000063924 & 0.000060187 \\
\hline
\end{tabular}

Table S2. Frequencies for target fluorinated ethanol and their related radicals at the $\mathrm{m} 062 \mathrm{x} / 6-31+\mathrm{g}(\mathrm{d}, \mathrm{p})$ level of theory $\left(\mathrm{cm}^{-1}\right)$.

\begin{tabular}{cccc}
\hline Target fluorinated ethanol & \multicolumn{3}{c}{ Frequencies $\left(\mathrm{cm}^{-1}\right)$} \\
\hline & 153.7115 & 318.4318 & 395.2338 \\
& 526.9463 & 879.0892 & 913.6087 \\
$\mathrm{CH}_{2} \mathrm{FCH}_{2} \mathrm{OH}$ & 1084.2017 & 1123.0654 & 1155.2746 \\
& 1227.6058 & 1278.1133 & 1382.4638 \\
& 1406.9769 & 1443.0414 & 1499.5695 \\
& 1503.3220 & 3040.8126 & 3082.2806 \\
& 3119.3826 & 3144.6188 & 3895.3665 \\
\hline
\end{tabular}


Continued

\begin{tabular}{|c|c|c|c|}
\hline \multirow{7}{*}{$\mathrm{CH}_{3} \mathrm{CHFOH}$} & 222.4821 & 381.0906 & 395.9003 \\
\hline & 476.6613 & 591.2739 & 882.7961 \\
\hline & 967.7343 & 1083.1639 & 1139.5797 \\
\hline & 1207.0636 & 1299.2263 & 1395.3640 \\
\hline & 1408.6162 & 1481.8193 & 1491.1530 \\
\hline & 1496.5668 & 3074.8833 & 3098.4238 \\
\hline & 3162.1117 & 3168.6137 & 3905.4122 \\
\hline \multirow{6}{*}{$\mathrm{C} \cdot \mathrm{HFCH}_{2} \mathrm{OH}$} & 117.2311 & 305.3799 & 387.2015 \\
\hline & 487.0844 & 672.8813 & 897.2987 \\
\hline & 1047.2118 & 1078.4074 & 1202.2847 \\
\hline & 1230.9543 & 1340.0387 & 1397.8007 \\
\hline & 1433.9762 & 1486.8167 & 3060.3820 \\
\hline & 3135.9012 & 3227.9702 & 3904.1459 \\
\hline \multirow{6}{*}{$\mathrm{CH}_{2} \mathrm{FCH} \bullet \mathrm{OH}$} & 136.3261 & 293.7121 & 372.9921 \\
\hline & 469.7867 & 588.3163 & 948.2198 \\
\hline & 976.5181 & 1064.6622 & 1237.5557 \\
\hline & 1261.6997 & 1324.3255 & 1380.0377 \\
\hline & 1477.7581 & 1517.2468 & 3089.0157 \\
\hline & 3148.4826 & 3209.7826 & 3921.1700 \\
\hline \multirow{6}{*}{$\mathrm{CH}_{2} \mathrm{FCH}_{2} \mathrm{O}$} & 138.8341 & 326.2587 & 461.7143 \\
\hline & 645.0346 & 900.2694 & 995.0829 \\
\hline & 095.1447 & 1132.5555 & 1209.7403 \\
\hline & 287.6953 & 1366.6408 & 1397.9225 \\
\hline & 436.1160 & 1499.8795 & 2978.3111 \\
\hline & 021.0190 & 3079.4857 & 3141.2839 \\
\hline \multirow{6}{*}{$\mathrm{CH}_{2} \cdot \mathrm{CHFOH}$} & 103.9492 & 354.4677 & 378.6019 \\
\hline & 464.4234 & 533.4936 & 615.0872 \\
\hline & 917.9447 & 973.1708 & 1082.8162 \\
\hline & 1204.3179 & 1292.9244 & 1375.2909 \\
\hline & 1421.9543 & 1501.7259 & 3093.4946 \\
\hline & 3176.3175 & 3301.9307 & 3902.4787 \\
\hline \multirow{6}{*}{$\mathrm{CH}_{3} \mathrm{CF} \cdot \mathrm{OH}$} & 183.0404 & 339.6091 & 381.8885 \\
\hline & 446.3670 & 566.0359 & 874.2810 \\
\hline & 992.1200 & 1063.5695 & 1140.5736 \\
\hline & 1302.2104 & 1363.7386 & 1442.6873 \\
\hline & 1477.4645 & 1485.2396 & 3031.7545 \\
\hline & 3128.3197 & 3174.6193 & 3874.3729 \\
\hline \multirow{6}{*}{$\mathrm{CH}_{3} \mathrm{CHFO} \bullet$} & 234.8451 & 359.2559 & 443.1211 \\
\hline & 572.8867 & 866.7266 & 929.6382 \\
\hline & 1040.2264 & 1088.1878 & 1158.3501 \\
\hline & 1216.4848 & 1326.7171 & 1391.8028 \\
\hline & 1484.8041 & 1492.1170 & 2997.0767 \\
\hline & 3077.4025 & 3170.0581 & 3179.9978 \\
\hline
\end{tabular}




\begin{tabular}{|c|c|c|c|}
\hline \multirow{7}{*}{$\mathrm{CH}_{2} \mathrm{FCHFOH}$} & 123.7297 & 253.3055 & 341.9812 \\
\hline & 442.3530 & 508.5501 & 585.7551 \\
\hline & 932.9529 & 1049.0131 & 1096.9489 \\
\hline & 1132.3163 & 1216.5809 & 1244.5656 \\
\hline & 1341.7047 & 1356.5333 & 1432.8601 \\
\hline & 1477.0191 & 1509.6783 & 3073.3252 \\
\hline & 3141.2603 & 3156.9890 & 3868.8911 \\
\hline \multirow{7}{*}{$\mathrm{CF}_{2} \mathrm{HCH}_{2} \mathrm{OH}$} & 261.5051 & 333.1831 & 429.7499 \\
\hline & 803.8978 & 905.9089 & 1069.3307 \\
\hline & 1113.2025 & 1153.4006 & 1280.2493 \\
\hline & 1377.1368 & 1403.4215 & 1433.7765 \\
\hline & 1493.5768 & 1500.2248 & 1525.4172 \\
\hline & 3032.2176 & 3044.0073 & 3104.6932 \\
\hline & 3125.0745 & 3137.9412 & 3910.0283 \\
\hline \multirow{7}{*}{$\mathrm{CH}_{3} \mathrm{CF}_{2} \mathrm{OH}$} & 213.0609 & 336.6797 & 378.8026 \\
\hline & 407.4641 & 552.4402 & 575.8400 \\
\hline & 584.5503 & 847.6087 & 959.8589 \\
\hline & 1000.3581 & 1135.7264 & 1204.1092 \\
\hline & 1301.5094 & 1390.3411 & 1483.7215 \\
\hline & 1488.5073 & 1492.2192 & 3086.0281 \\
\hline & 3175.9594 & 3186.0332 & 3866.1356 \\
\hline \multirow{6}{*}{$\mathrm{CH}_{2} \mathrm{FC} \cdot \mathrm{FOH}$} & 109.4115 & 244.9232 & 388.6686 \\
\hline & 436.4869 & 472.3557 & 575.6453 \\
\hline & 917.0734 & 1055.3780 & 1102.3841 \\
\hline & 1135.9138 & 1244.9454 & 1336.2661 \\
\hline & 1365.0202 & 1448.3398 & 1502.9979 \\
\hline & 3049.1641 & 3148.5710 & 3871.9507 \\
\hline \multirow{6}{*}{$\mathrm{C} \cdot \mathrm{HFCFHOH}$} & 107.9351 & 257.2006 & 353.4210 \\
\hline & 416.6962 & 546.9238 & 646.1233 \\
\hline & 765.4796 & 916.4141 & 991.0405 \\
\hline & 1161.1762 & 1227.5285 & 1327.6897 \\
\hline & 1356.7224 & 1403.4567 & 1473.0076 \\
\hline & 3148.9051 & 3254.0619 & 3863.4809 \\
\hline \multirow{6}{*}{$\mathrm{CH}_{2} \mathrm{FCHFO} \bullet$} & 122.5173 & 253.1428 & 406.6622 \\
\hline & 426.8055 & 595.6816 & 922.4453 \\
\hline & 981.5646 & 1062.7404 & 1130.5318 \\
\hline & 1159.6593 & 1193.8858 & 1264.5276 \\
\hline & 1306.1655 & 1411.2155 & 1509.8582 \\
\hline & 3020.9800 & 3106.4335 & 3176.7438 \\
\hline \multirow{6}{*}{$\mathrm{CHF}_{2} \mathrm{C} \cdot \mathrm{HOH}$} & 102.5287 & 255.5062 & 307.6957 \\
\hline & 498.7454 & 531.8669 & 588.6407 \\
\hline & 776.4400 & 943.7162 & 1040.6628 \\
\hline & 1117.9847 & 1203.9780 & 1346.8684 \\
\hline & 1361.3396 & 1407.7937 & 1499.6505 \\
\hline & 3165.5275 & 3252.1304 & 3846.9473 \\
\hline
\end{tabular}




\section{Continued}

\begin{tabular}{|c|c|c|c|}
\hline \multirow{6}{*}{$\mathrm{C} \cdot \mathrm{F}_{2} \mathrm{CH}_{2} \mathrm{OH}$} & 111.2059 & 240.3480 & 355.7645 \\
\hline & 439.8572 & 476.0825 & 590.2258 \\
\hline & 889.8898 & 1035.2006 & 1136.6153 \\
\hline & 1208.8861 & 1259.6672 & 1294.5087 \\
\hline & 1380.7274 & 1441.8811 & 1496.7631 \\
\hline & 3013.5503 & 3147.9499 & 3898.0279 \\
\hline \multirow{6}{*}{$\mathrm{CHF}_{2} \mathrm{CH}_{2} \mathrm{O}$ - } & 128.4545 & 257.5539 & 433.4387 \\
\hline & 494.0758 & 586.0455 & 917.5567 \\
\hline & 1096.8914 & 1139.6268 & 1157.8405 \\
\hline & 1232.6325 & 1307.2674 & 1385.1305 \\
\hline & 1404.8759 & 1510.7673 & 1859.0406 \\
\hline & 3031.4094 & 3120.2437 & 3174.3851 \\
\hline \multirow{6}{*}{$\mathrm{CH}_{2} \cdot \mathrm{CF}_{2} \mathrm{OH}$} & 90.5157 & 313.8094 & 380.5941 \\
\hline & 392.3412 & 492.4899 & 547.1101 \\
\hline & 593.9731 & 597.4030 & 869.8795 \\
\hline & 960.9120 & 1070.4557 & 1131.1978 \\
\hline & 1308.1224 & 1403.4875 & 1499.7334 \\
\hline & 3211.2694 & 3342.0724 & 3863.9788 \\
\hline \multirow{6}{*}{$\mathrm{CH}_{3} \mathrm{CF}_{2} \mathrm{O} \cdot$} & 168.6127 & 340.9654 & 360.5945 \\
\hline & 534.8395 & 551.7060 & 557.0587 \\
\hline & 846.1077 & 915.5633 & 974.0145 \\
\hline & 1162.5612 & 1220.2174 & 1281.3277 \\
\hline & 1397.7811 & 1466.2734 & 1488.6608 \\
\hline & 3077.0770 & 3170.5354 & 3190.3722 \\
\hline
\end{tabular}


Table S3. Moment of inertia for target fluorinated ethanol and their related radicals at the $\mathrm{m} 062 \mathrm{x} / 6-31+\mathrm{g}(\mathrm{d}, \mathrm{p})$ level of theory (GHZ).

\begin{tabular}{|c|c|c|c|}
\hline \multirow{2}{*}{$\begin{array}{l}\text { target fluorinated ethanol } \\
\qquad \mathrm{CH}_{2} \mathrm{FCH}_{2} \mathrm{OH}\end{array}$} & \multicolumn{3}{|c|}{ Moment of inertia(GHZ) } \\
\hline & 15.7788859 & 5.5493007 & 4.6093694 \\
\hline $\mathrm{CH}_{3} \mathrm{CHFOH}$ & 10.0766267 & 8.3371985 & 5.1680915 \\
\hline $\mathrm{C} \cdot \mathrm{HFCH}_{2} \mathrm{OH}$ & 18.77997 & 5.13190 & 4.51693 \\
\hline $\mathrm{CH}_{2} \mathrm{FCH} \bullet \mathrm{OH}$ & 23.2409534 & 4.5476319 & 4.3485670 \\
\hline $\mathrm{CH}_{2} \mathrm{FCH}_{2} \mathrm{O}$. & 18.24036 & 5.23748 & 4.59279 \\
\hline $\mathrm{CH}_{2} \cdot \mathrm{CHFOH}$ & 10.3760339 & 9.1250064 & 5.3599040 \\
\hline $\mathrm{CH}_{3} \mathrm{CF} \bullet \mathrm{OH}$ & 10.20355 & 9.05876 & 5.07651 \\
\hline $\mathrm{CH}_{3} \mathrm{CHFO} \bullet$ & 10.7702750 & 8.5850705 & 5.3185779 \\
\hline $\mathrm{CH}_{2} \mathrm{FCHFOH}$ & 9.4161023 & 3.5112856 & 2.7600267 \\
\hline $\mathrm{CF}_{2} \mathrm{HCH}_{2} \mathrm{OH}$ & 34.8864548 & 9.2467926 & 8.1783038 \\
\hline $\mathrm{CH}_{3} \mathrm{CF}_{2} \mathrm{OH}$ & 9.4161023 & 3.5112856 & 2.7600267 \\
\hline $\mathrm{CH}_{2} \mathrm{FC} \cdot \mathrm{FOH}$ & 9.7798799 & 3.5506736 & 2.7289504 \\
\hline $\mathrm{C} \cdot \mathrm{HFCFHOH}$ & 9.5295647 & 3.7425748 & 2.9008390 \\
\hline $\mathrm{CH}_{2} \mathrm{FCHFO} \bullet$ & 10.2331738 & 3.5038934 & 2.8019184 \\
\hline $\mathrm{CHF}_{2} \mathrm{C} \cdot \mathrm{HOH}$ & 9.1000423 & 3.8971695 & 3.0141244 \\
\hline $\mathrm{C} \cdot \mathrm{F}_{2} \mathrm{CH}_{2} \mathrm{OH}$ & 9.6401202 & 3.8114853 & 2.8520087 \\
\hline $\mathrm{CHF}_{2} \mathrm{CH}_{2} \mathrm{O}$ • & 9.21258 & 3.85327 & 2.93914 \\
\hline $\mathrm{CH}_{2} \cdot \mathrm{CF}_{2} \mathrm{OH}$ & 5.7024545 & 5.2730634 & 5.1759614 \\
\hline $\mathrm{CH}_{3} \mathrm{CF}_{2} \mathrm{O} \bullet$ & 5.9149594 & 5.2219748 & 5.0070937 \\
\hline
\end{tabular}

Optimized Geometries for target fluorinated ethanol and their related radicals at the $\mathrm{m} 062 \mathrm{x} / 6-31+\mathrm{g}(\mathrm{d}, \mathrm{p})$ level of theory

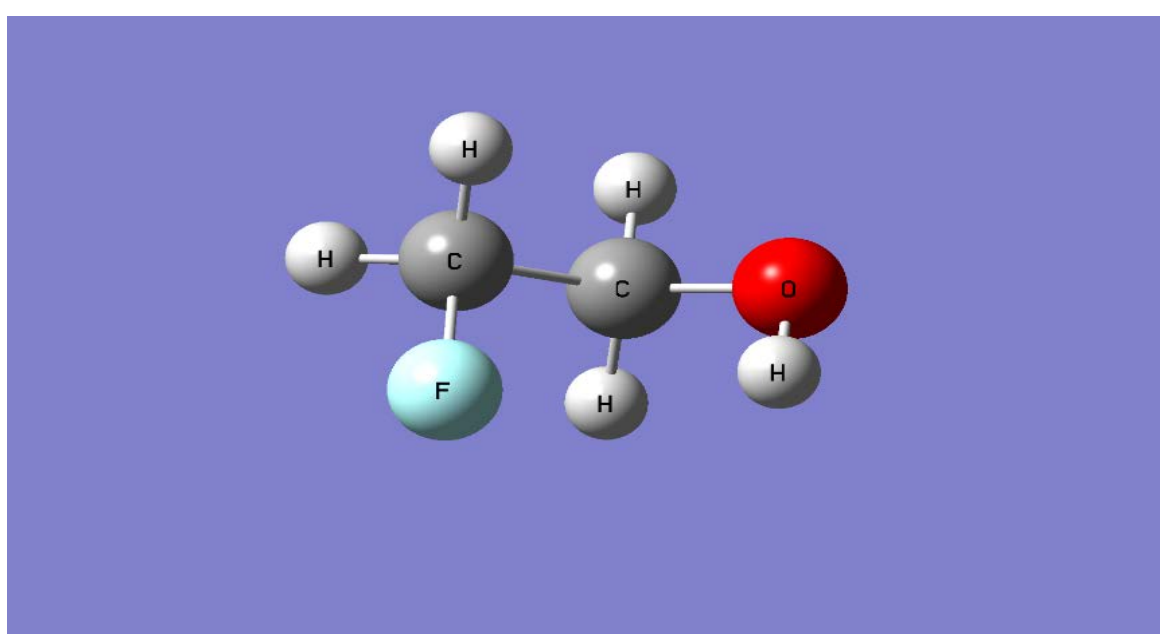

Figure S1. Optimized Geometry for $\mathrm{CH} 2 \mathrm{FCH} 2 \mathrm{OH}$ at the m062x/6-31 + g (d, p) level of theory. 


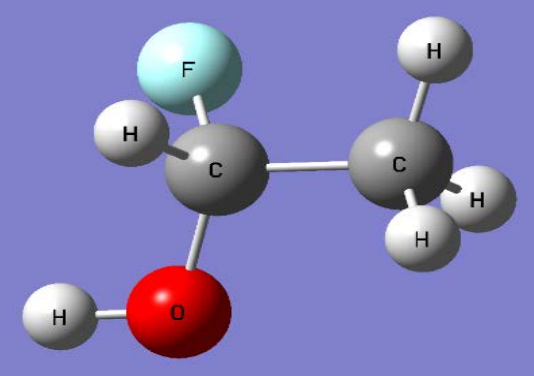

Figure S2. Optimized Geometry for $\mathrm{CH}_{3} \mathrm{CHFOH}$ at the m062x/6-31 $+\mathrm{g}(\mathrm{d}, \mathrm{p})$ level of theory.

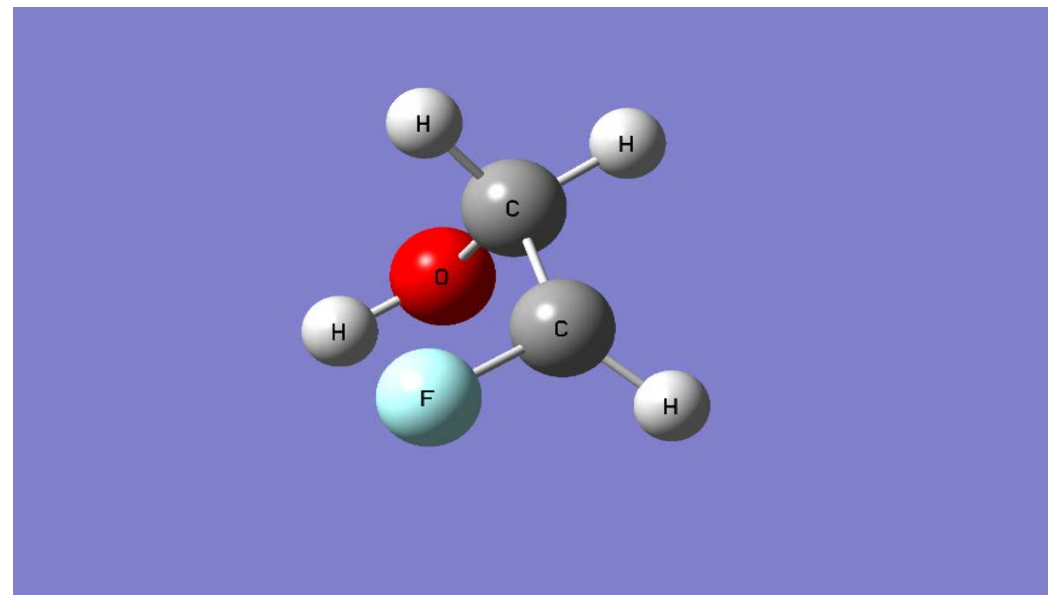

Figure S3. Optimized Geometry for $\mathrm{C} \cdot \mathrm{HFCH}_{2} \mathrm{OH}$ at the $\mathrm{m} 062 \mathrm{x} / 6-31+\mathrm{g}(\mathrm{d}, \mathrm{p})$ level of theory.

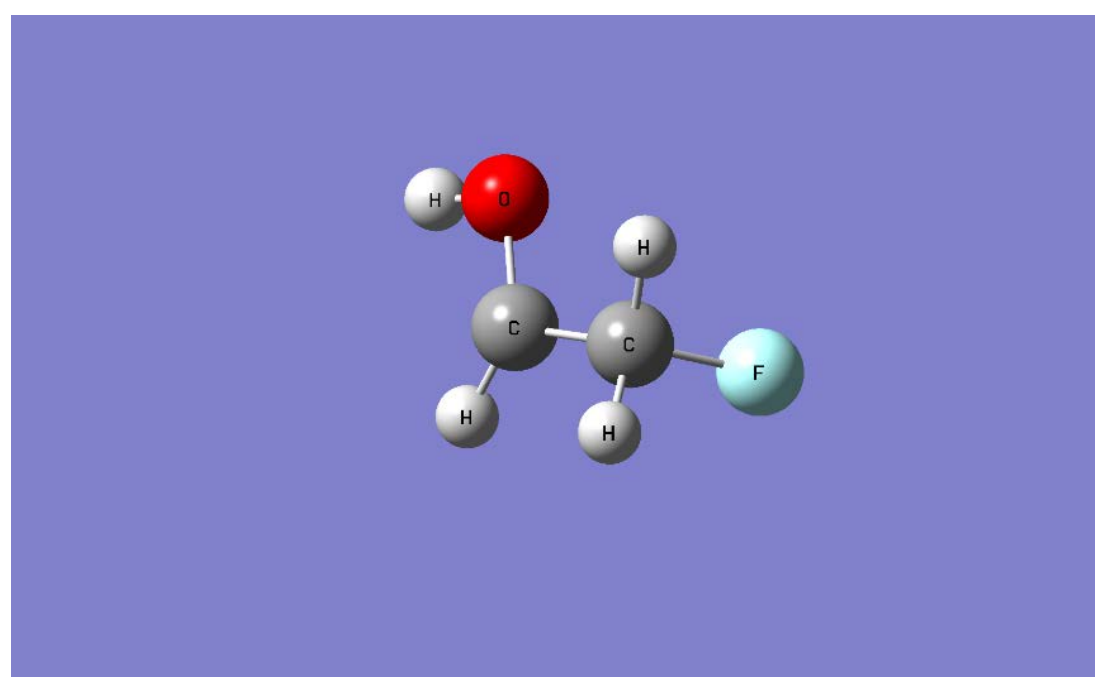

Figure S4. Optimized Geometry for $\mathrm{CH}_{2} \mathrm{FCH} \bullet \mathrm{OH}$ at the $\mathrm{m} 062 \mathrm{x} / 6-31+\mathrm{g}(\mathrm{d}, \mathrm{p})$ level of theory. 


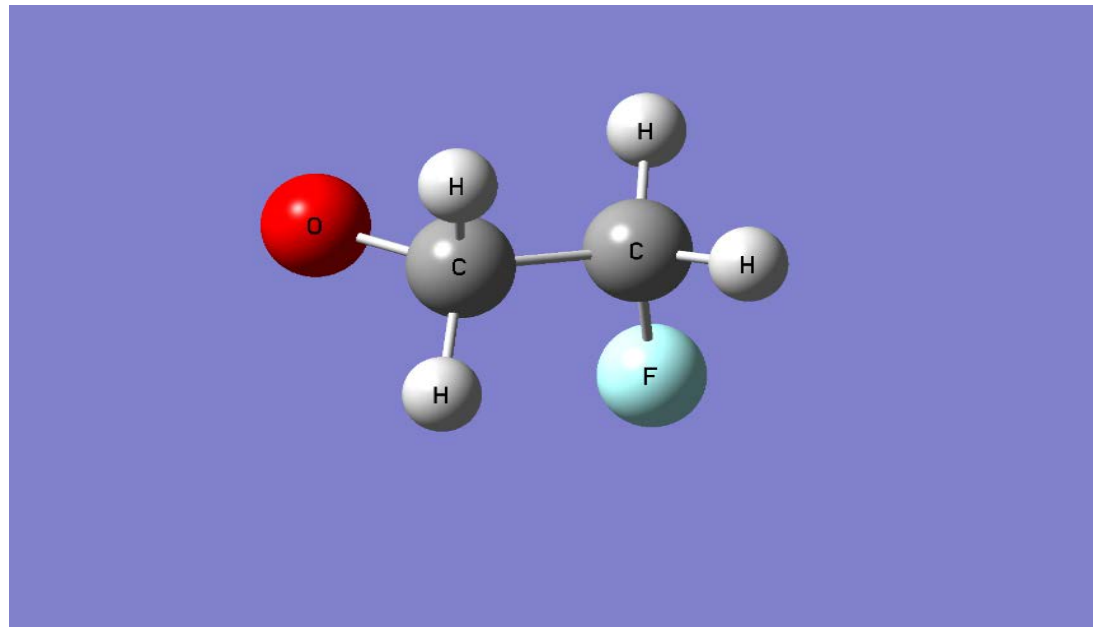

Figure S5. Optimized Geometry for $\mathrm{CH}_{2} \mathrm{FCH}_{2} \mathrm{O}$ - at the $\mathrm{m} 062 \mathrm{x} / 6-31+\mathrm{g}(\mathrm{d}, \mathrm{p})$ level of theory.

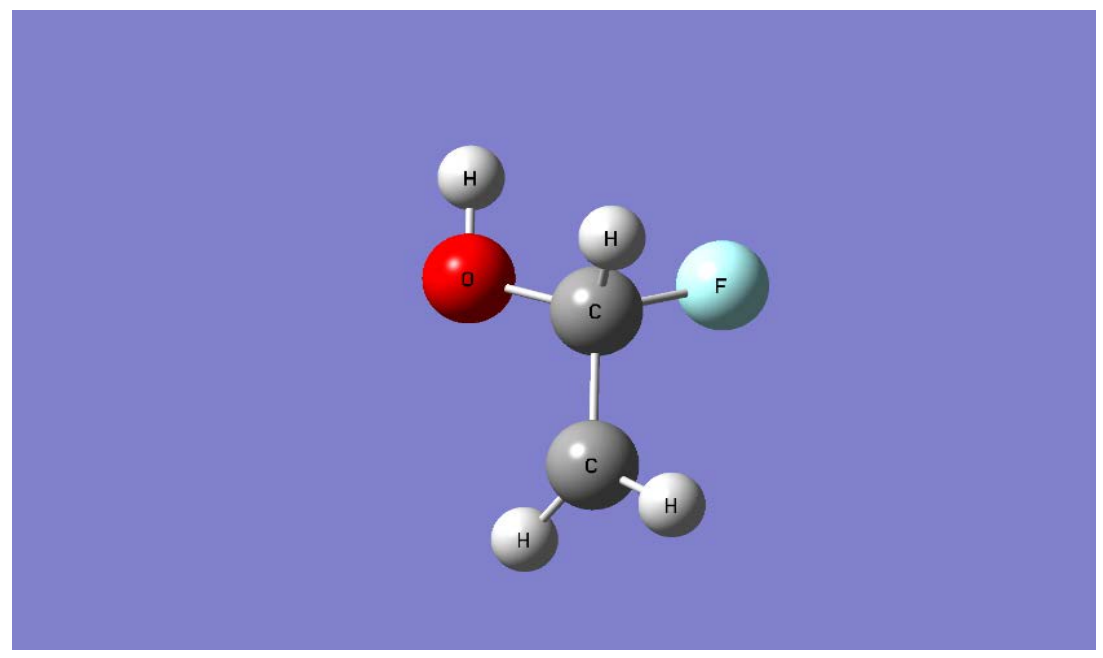

Figure S6. Optimized Geometry for $\mathrm{CH}_{2} \bullet \mathrm{CHFOH}$ at the m062x/6-31 $+\mathrm{g}(\mathrm{d}, \mathrm{p})$ level of theory.

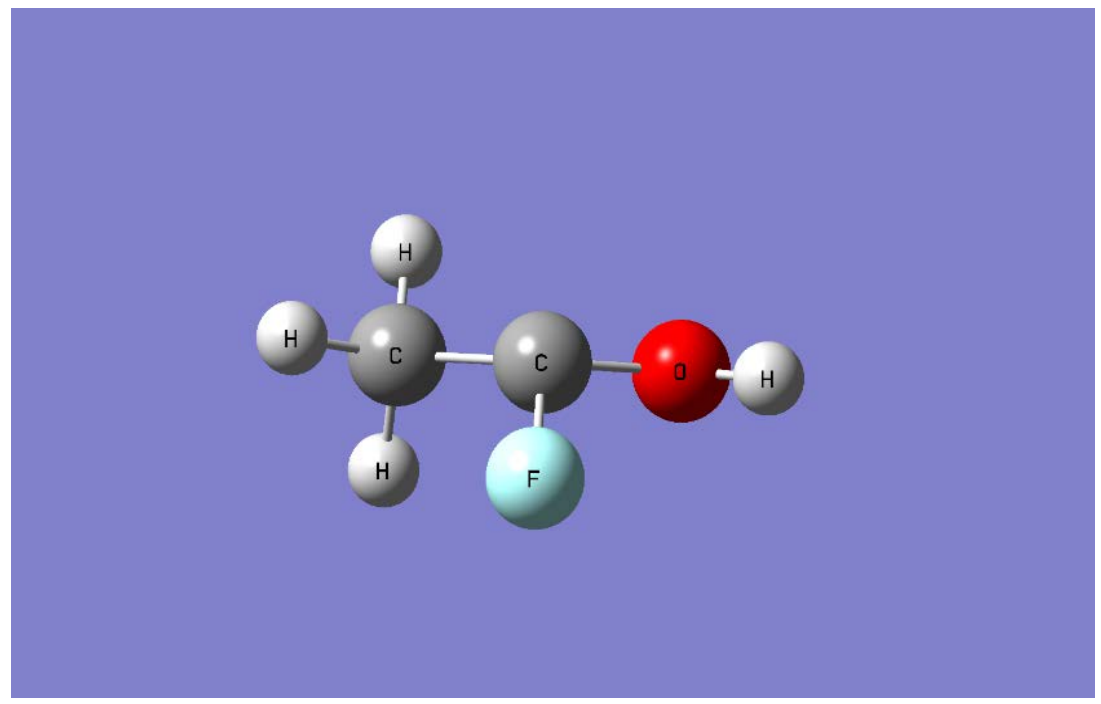

Figure S7. Optimized Geometry for $\mathrm{CH}_{3} \mathrm{CF} \bullet \mathrm{OH}$ at the $\mathrm{m} 062 \mathrm{x} / 6-31+\mathrm{g}(\mathrm{d}, \mathrm{p})$ level of theory. 


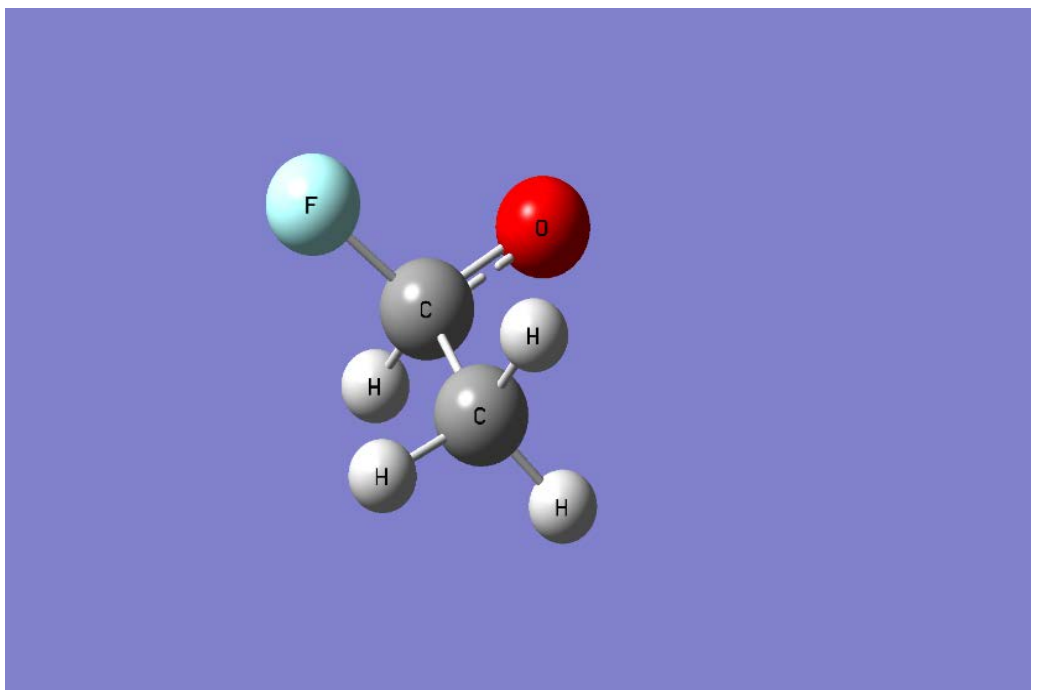

Figure S8. Optimized Geometry for $\mathrm{CH}_{3} \mathrm{CHFO}$ - at the m062x/6-31 + g (d, p) level of theory.

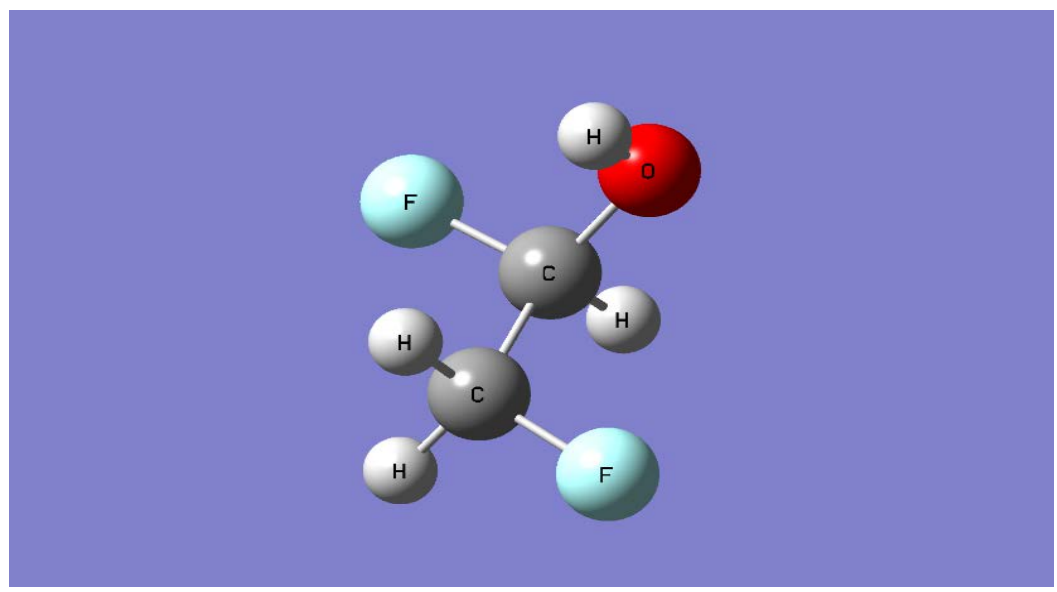

Figure S9. Optimized Geometry for $\mathrm{CH}_{2} \mathrm{FCHFOH}$ at the $\mathrm{m} 062 \mathrm{x} / 6-31+\mathrm{g}(\mathrm{d}, \mathrm{p})$ level of theory.

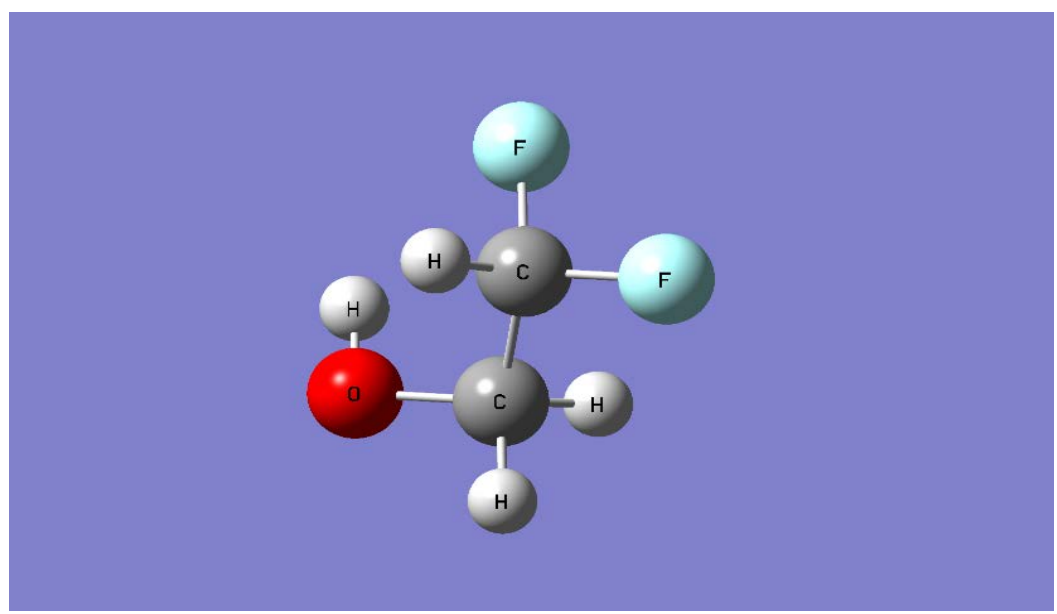

Figure S10. Optimized Geometry for $\mathrm{CF}_{2} \mathrm{HCH}_{2} \mathrm{OH}$ at the m062x/6-31 $+\mathrm{g}(\mathrm{d}, \mathrm{p})$ level of theory. 


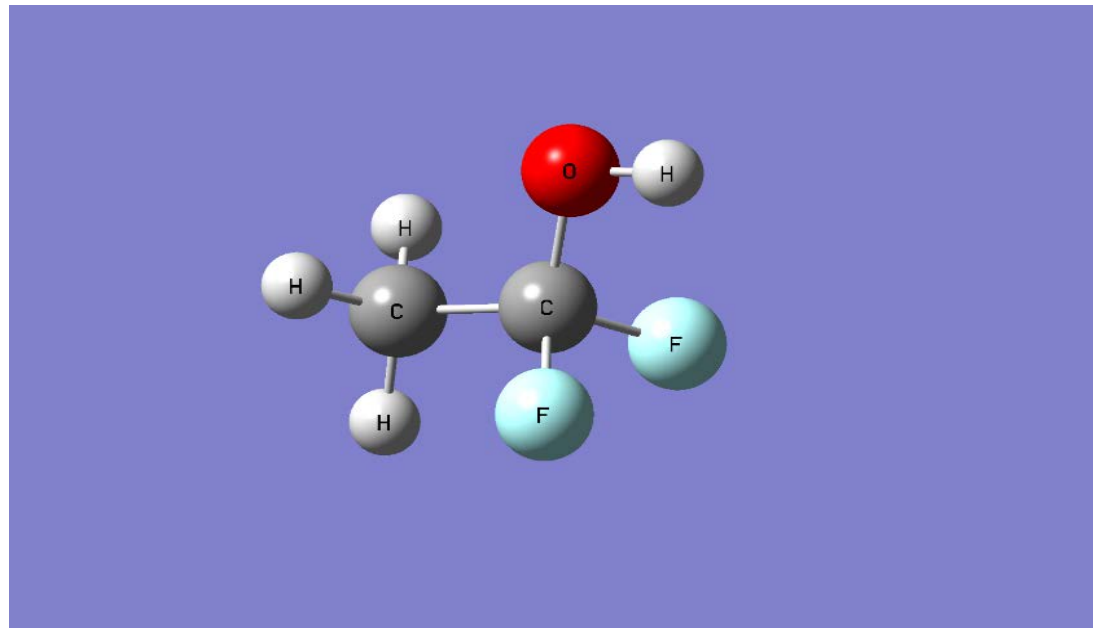

Figure S11. Optimized Geometry for $\mathrm{CH}_{3} \mathrm{CF}_{2} \mathrm{OH}$ at the $\mathrm{m} 062 \mathrm{x} / 6-31+\mathrm{g}(\mathrm{d}, \mathrm{p})$ level of theory.

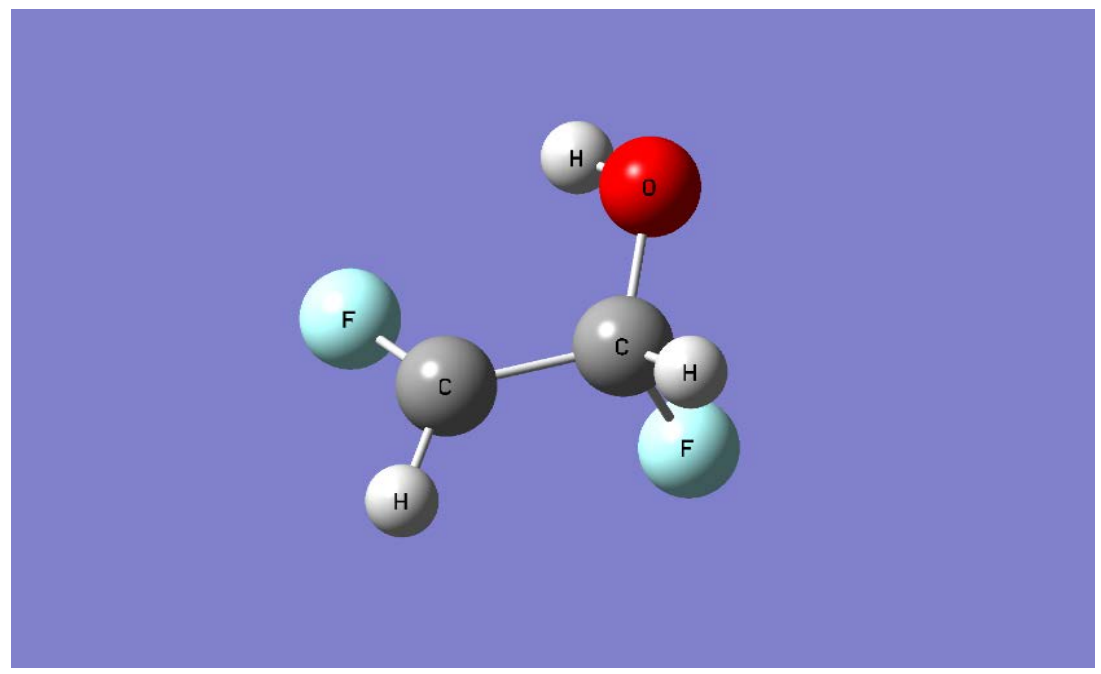

Figure S12. Optimized Geometry for C.HFCFHOH at the m062x/6-31 + g (d, p) level of theory.

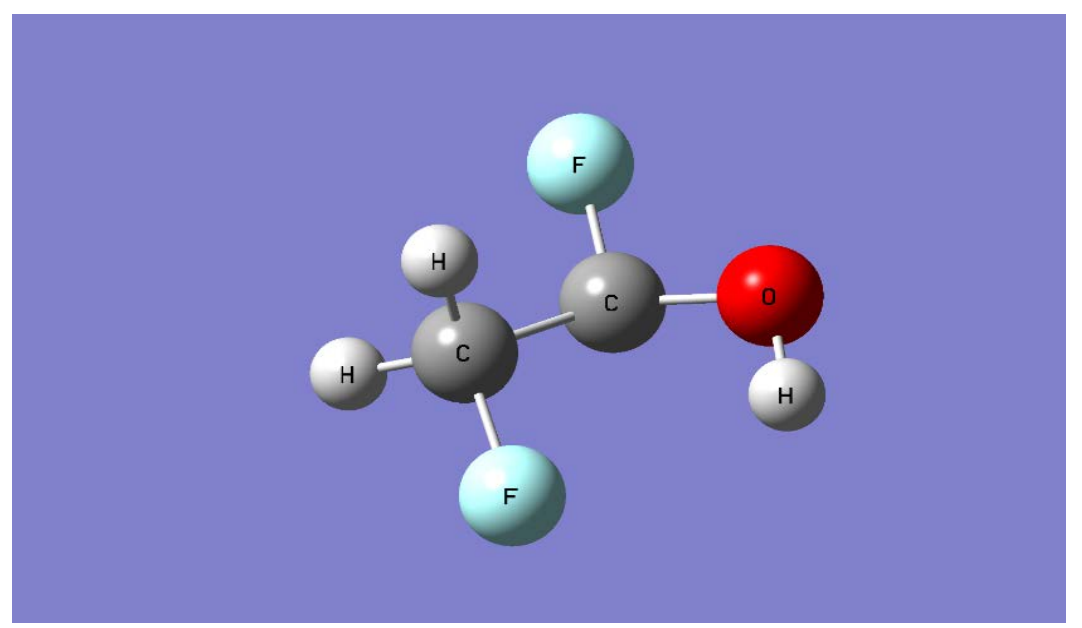

Figure S13. Optimized Geometry for $\mathrm{CH}_{2} \mathrm{FC} \cdot \mathrm{FOH}$ at the $\mathrm{m} 062 \mathrm{x} / 6-31+\mathrm{g}(\mathrm{d}, \mathrm{p})$ level of theory. 


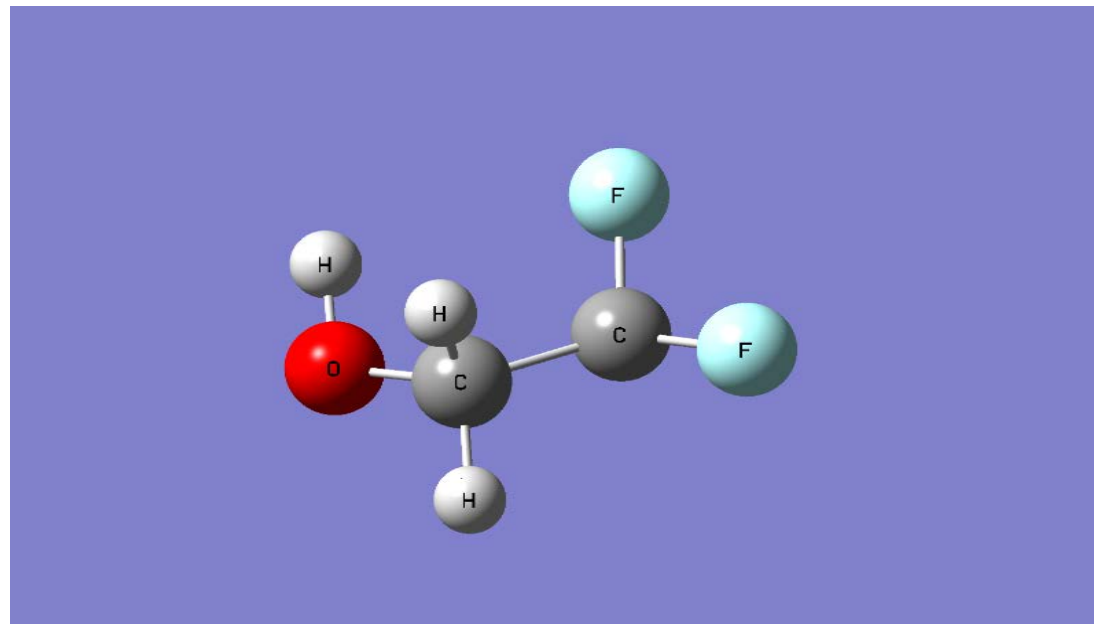

Figure S14. Optimized Geometry for $\mathrm{C}_{\bullet} \mathrm{F}_{2} \mathrm{CH}_{2} \mathrm{OH}$ at the $\mathrm{m} 062 \mathrm{x} / 6-31+\mathrm{g}(\mathrm{d}, \mathrm{p})$ level of theory.

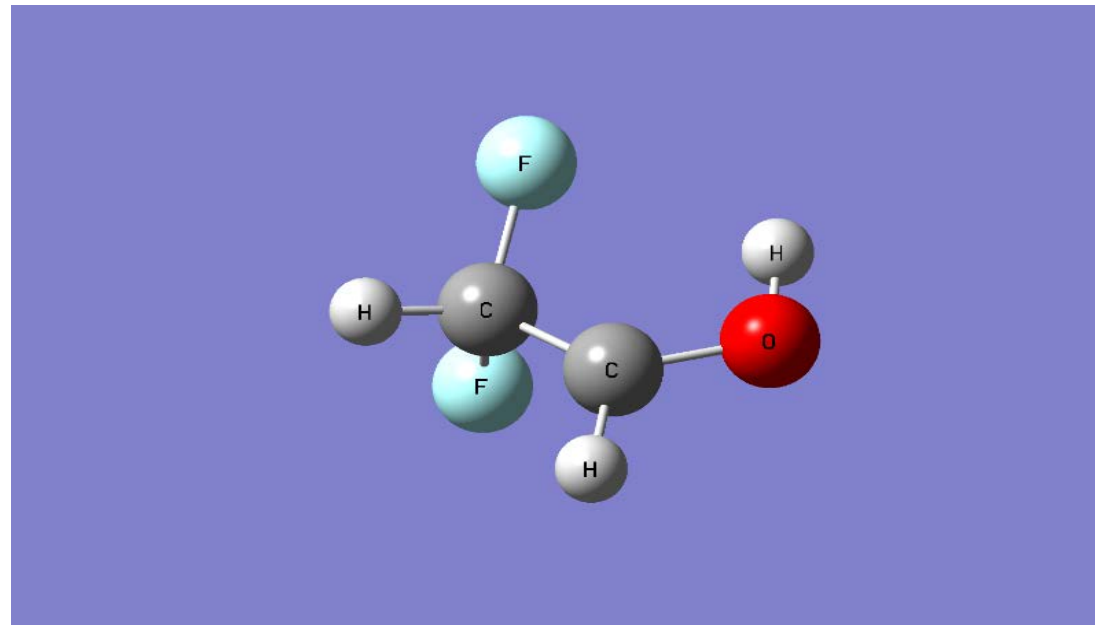

Figure S15. Optimized Geometry for $\mathrm{CHF}_{2} \mathrm{C} \cdot \mathrm{HOH}$ at the m062x/6-31 + g (d, p) level of theory.

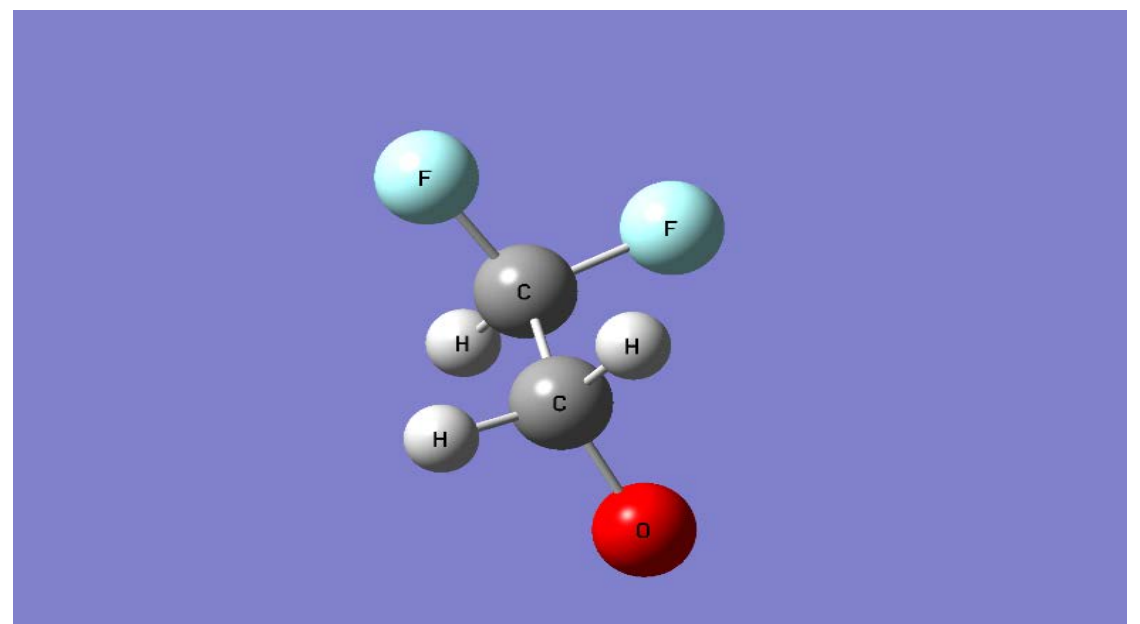

Figure S16. Optimized Geometry for $\mathrm{CHF}_{2} \mathrm{CH}_{2} \mathrm{O}$ - at the m062x/6-31 + g (d, p) level of theory. 


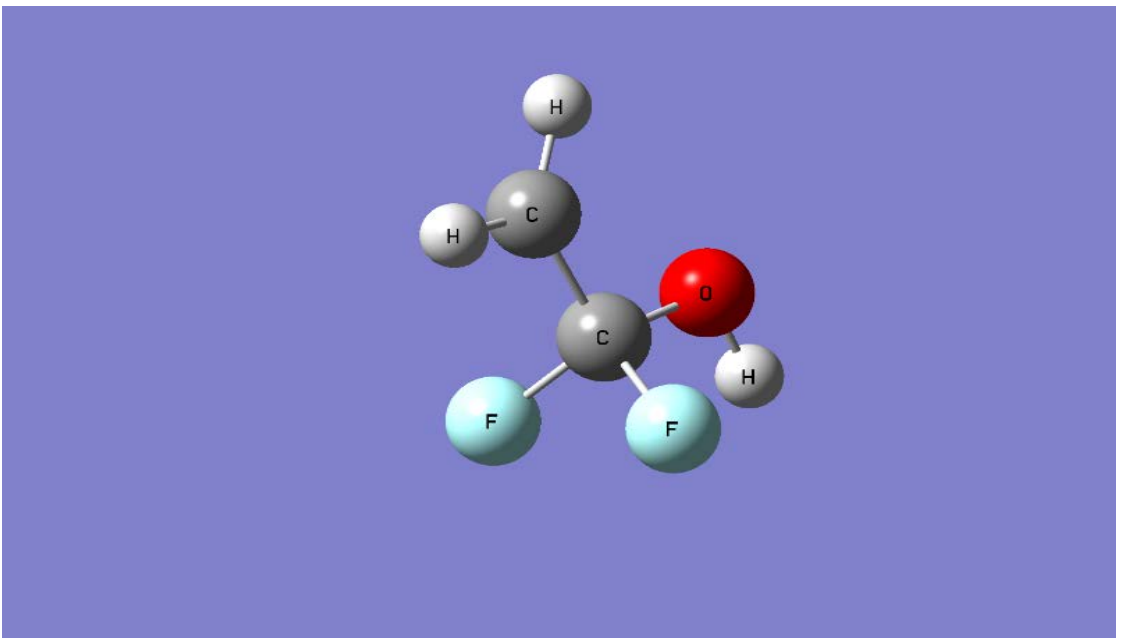

Figure S17. Optimized Geometry for $\mathrm{CH}_{2} \cdot \mathrm{CF}_{2} \mathrm{OH}$ at the $\mathrm{m} 062 \mathrm{x} / 6-31+\mathrm{g}(\mathrm{d}, \mathrm{p})$ level of theory.

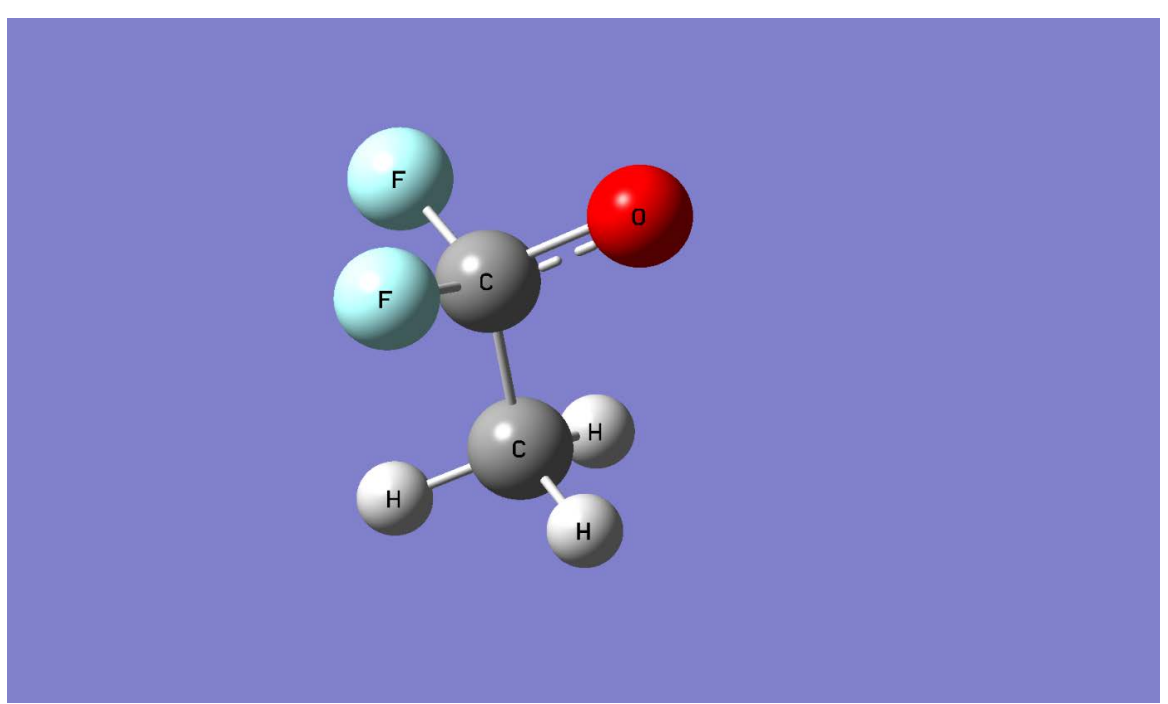

Figure S18. Optimized Geometry for $\mathrm{CH}_{3} \mathrm{CF}_{2} \mathrm{O} \bullet$ at the m062x/6-31 + g (d, p) level of theory. 

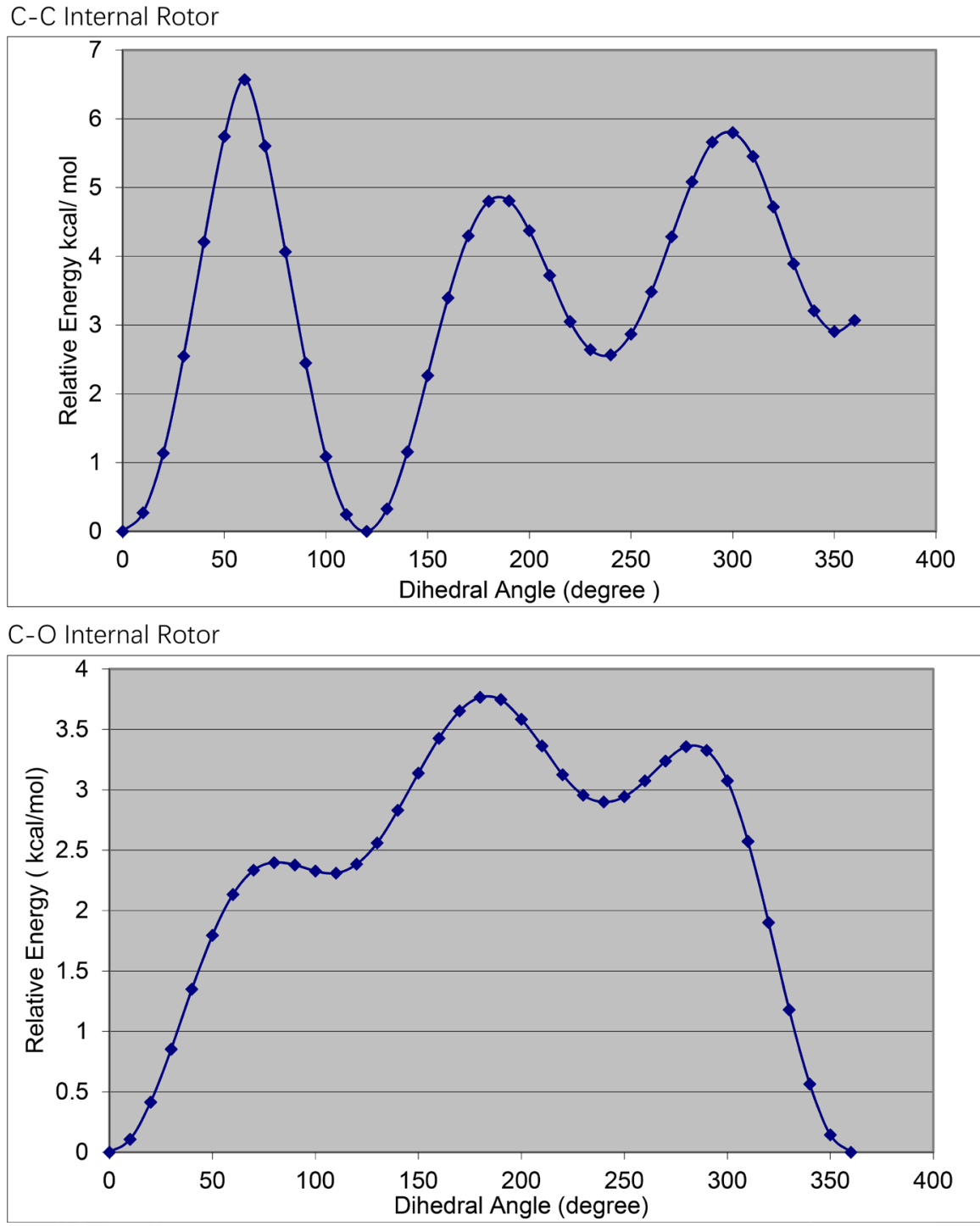

Figure S19. Potential energy profile of $\mathrm{C}-\mathrm{C}$ and $\mathrm{C}-\mathrm{O}$ internal rotors for $\mathrm{CH}_{2} \mathrm{FCH}_{2} \mathrm{OH}$. The solid lines indicate Fourier series expansion. 


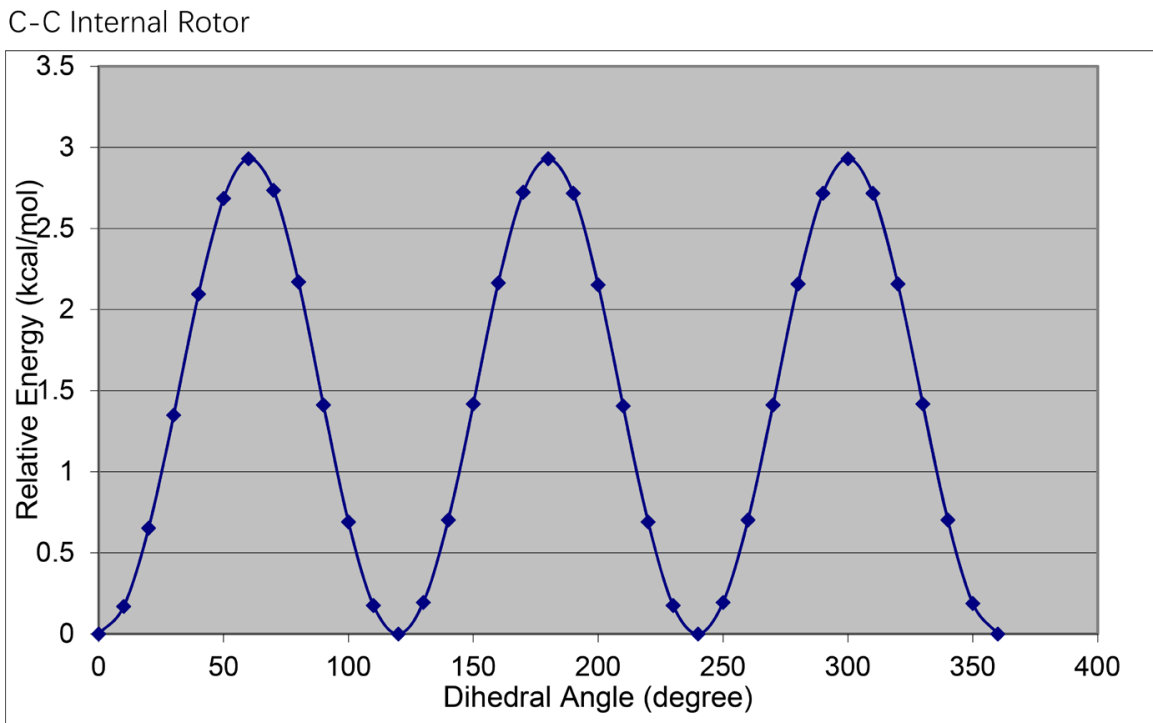

C-O Internal Rotor

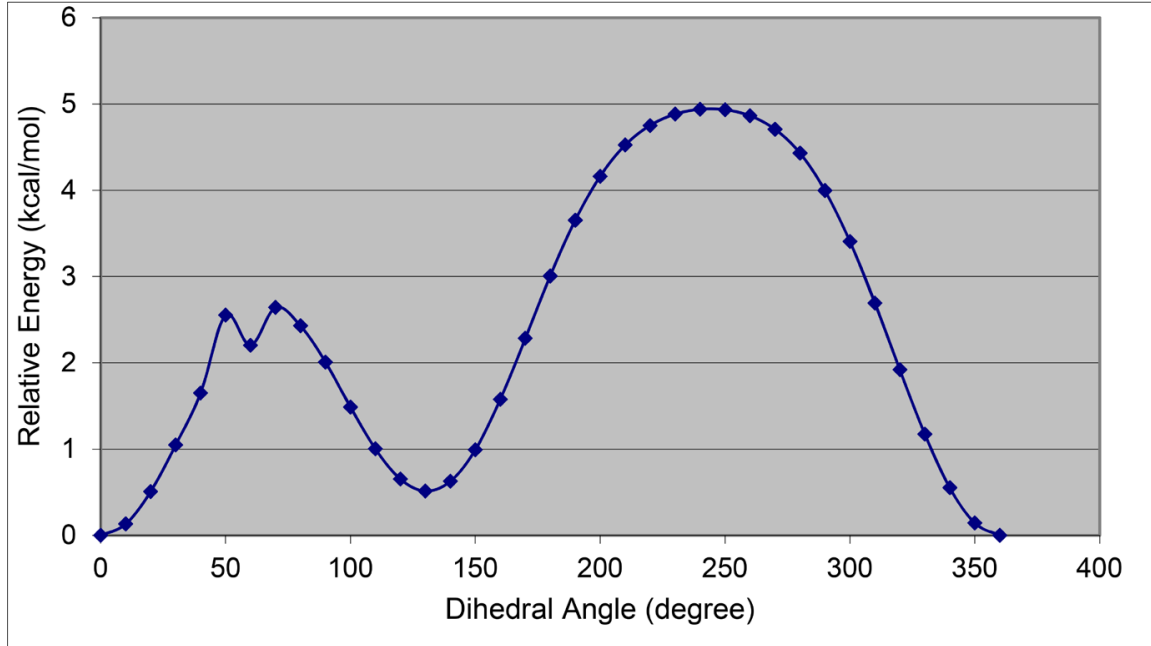

Figure S20. Potential energy profile of C-C and C-O internal rotors for $\mathrm{CH}_{3} \mathrm{CHFOH}$. The solid lines indicate Fourier series expansion. 
C-C Internal Rotor

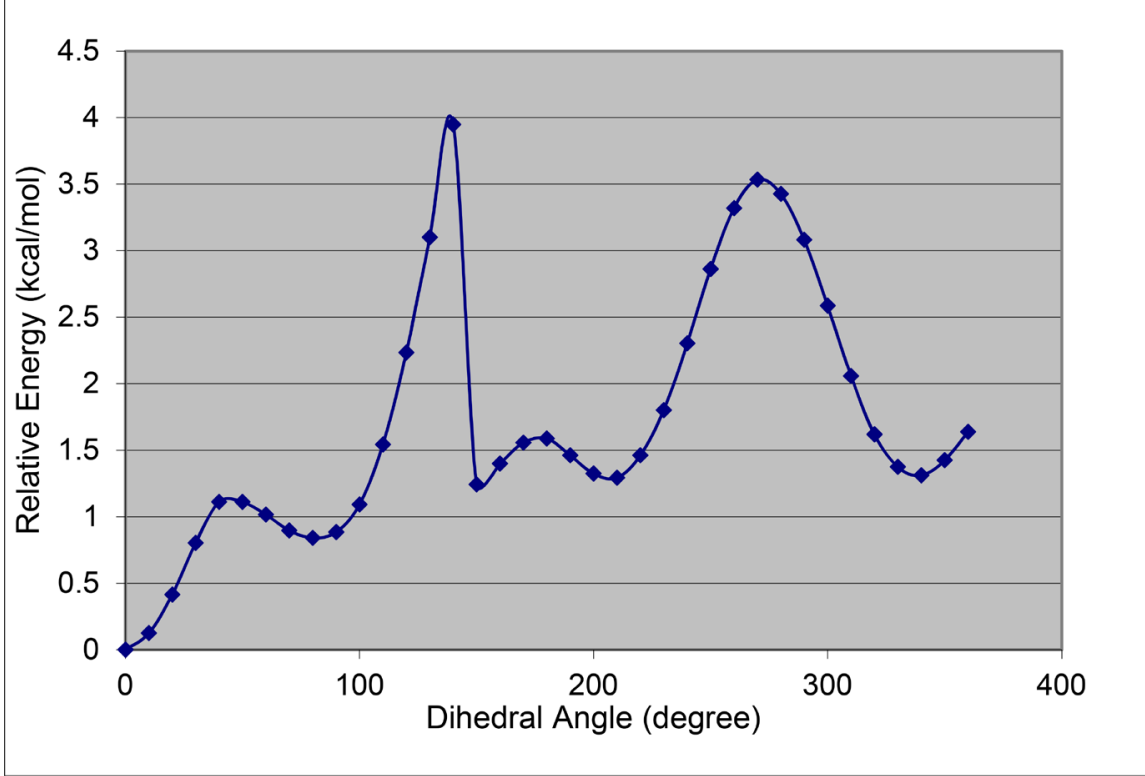

C-O Internal Rotor

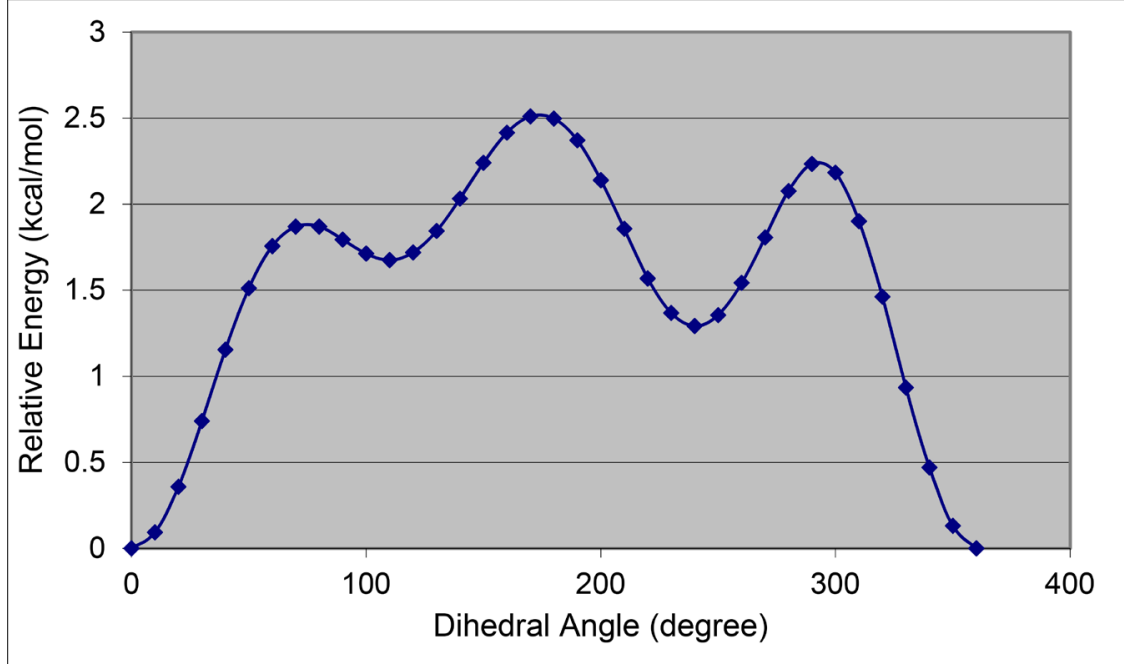

Figure S21. Potential energy profile of C-C and C-O internal rotors for C• $\mathrm{HFCH}_{2} \mathrm{OH}$. The solid lines indicate Fourier series expansion. 
C-C Internal Rotor
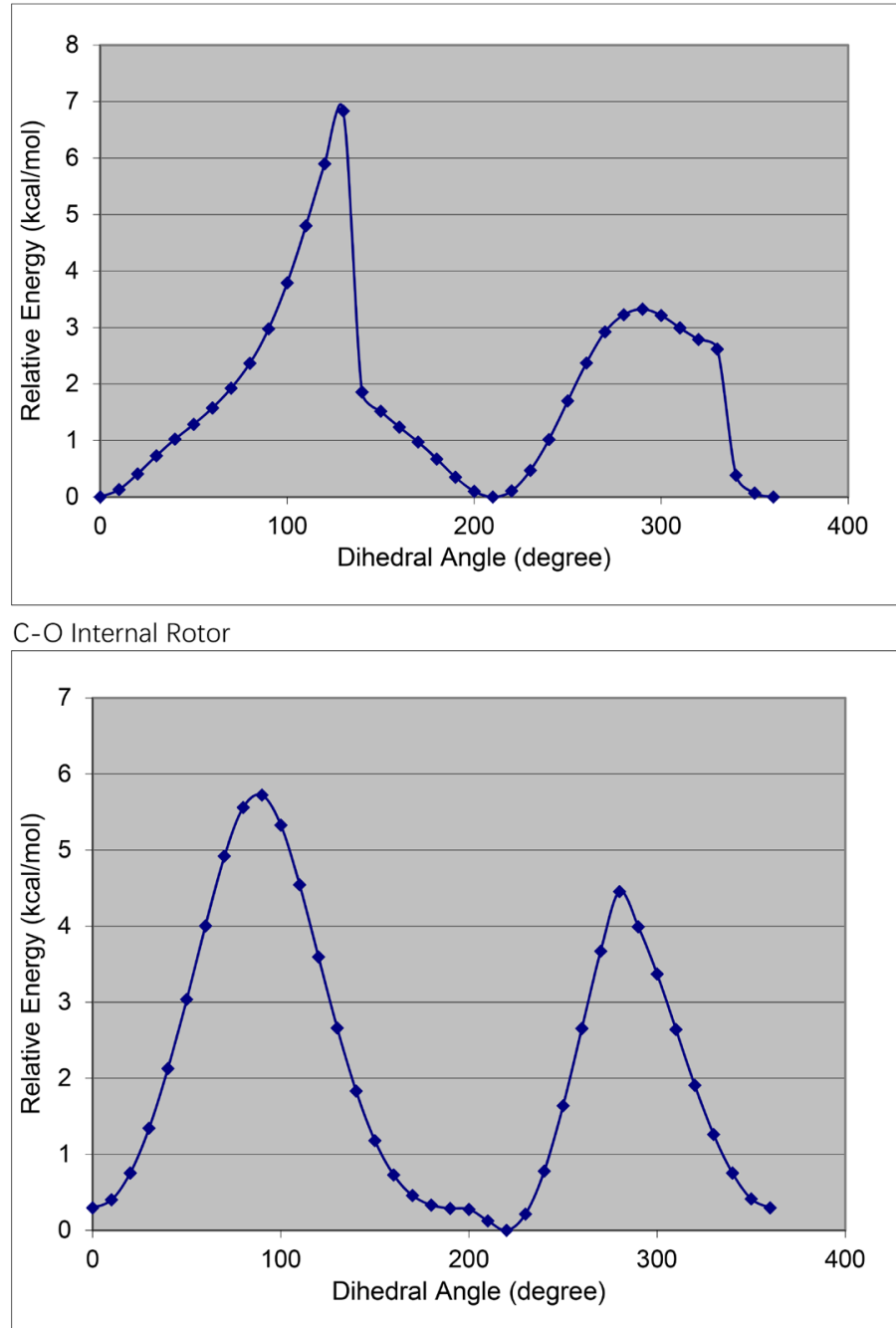

Figure S22. Potential energy profile of $\mathrm{C}-\mathrm{C}$ and $\mathrm{C}-\mathrm{O}$ internal rotors for $\mathrm{CH}_{2} \mathrm{FCH} \bullet \mathrm{OH}$. The solid lines indicate Fourier series expansion.

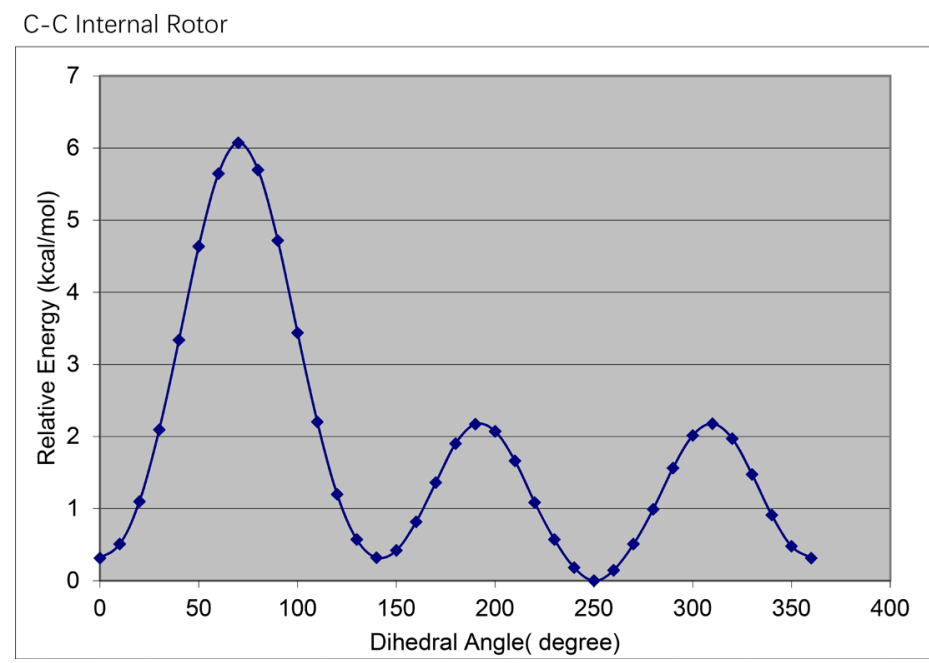

Figure S23. Potential energy profile of $\mathrm{C}-\mathrm{C}$ and $\mathrm{C}-\mathrm{O}$ internal rotors for $\mathrm{CH}_{2} \mathrm{FCH}_{2} \mathrm{O}$ •. The solid lines indicate Fourier series expansion. 


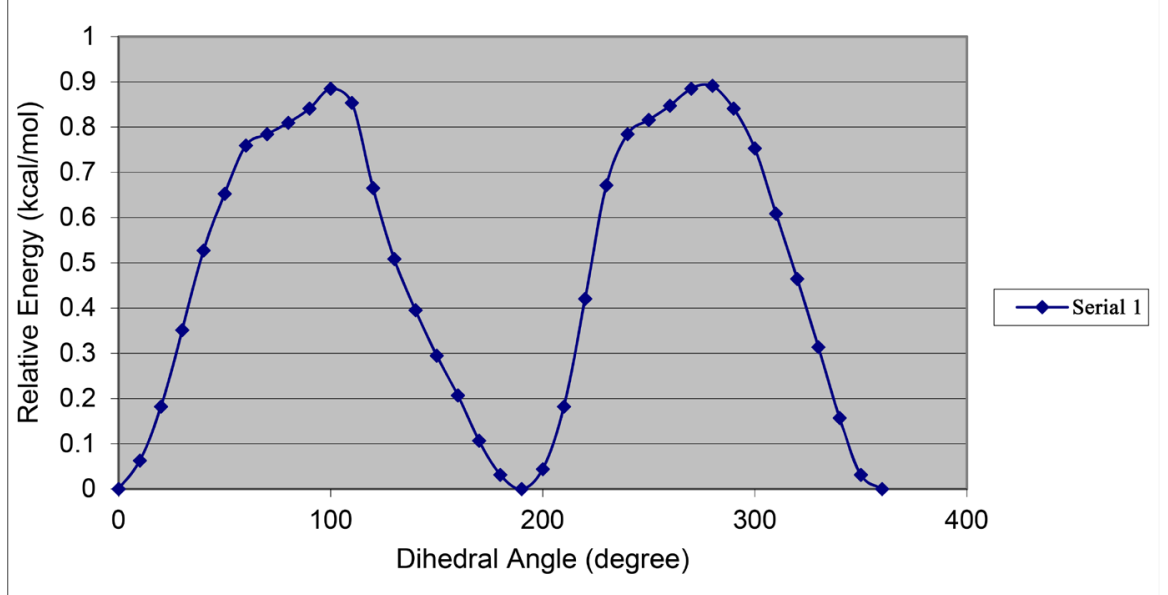

\section{C-O Internal Rotor}

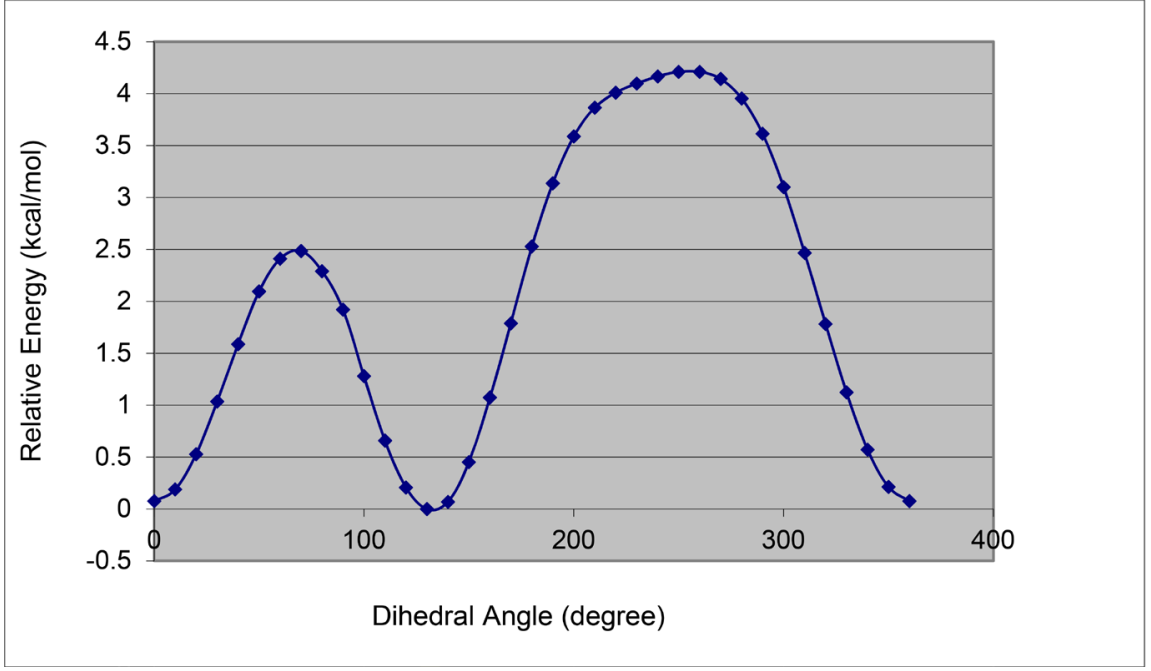

Figure S24. Potential energy profile of C-C and C-O internal rotors for $\mathrm{CH}_{2} \bullet \mathrm{CHFOH}$. The solid lines indicate Fourier series expansion. 


\section{C-C Internal Rotor}

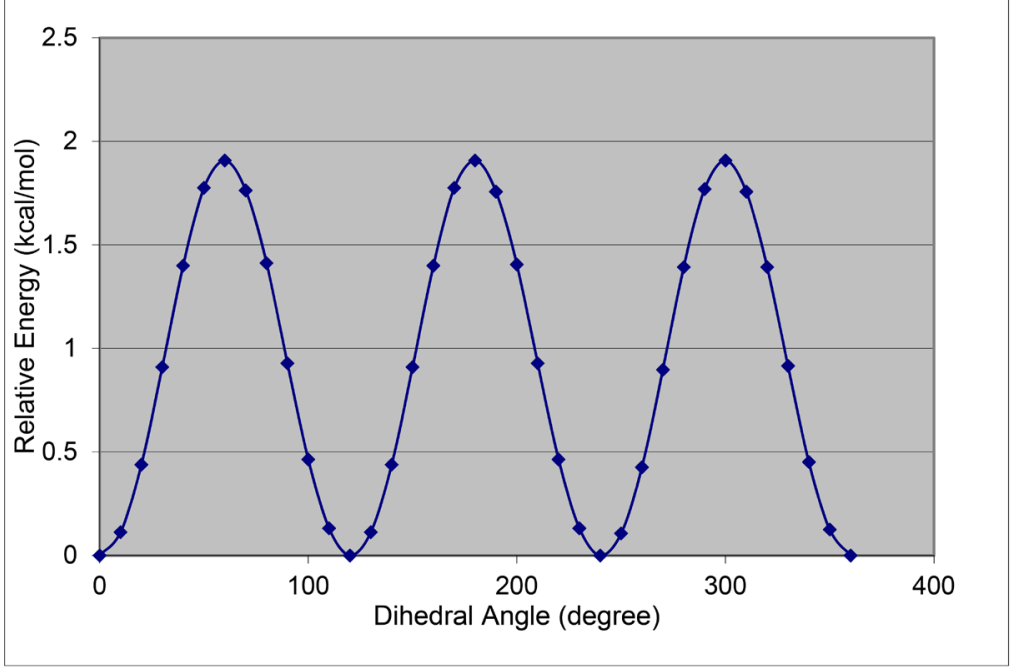

\section{C-O Internal Rotor}

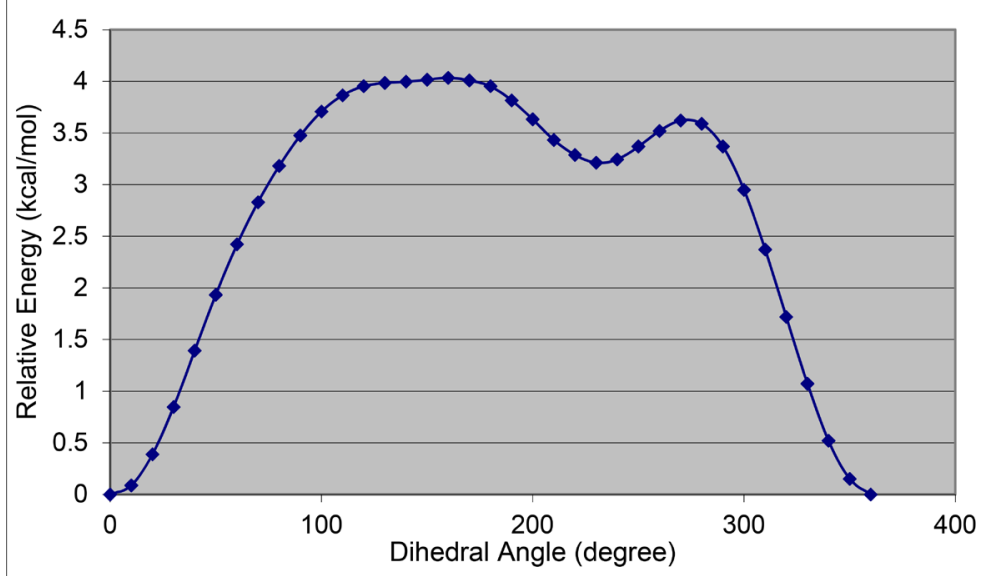

Figure S25. Potential energy profile of C-C and C-O internal rotors for $\mathrm{CH}_{3} \mathrm{CF} \bullet \mathrm{OH}$.The solid lines indicate Fourier series expansion.

\section{C-C Internal Rotor}

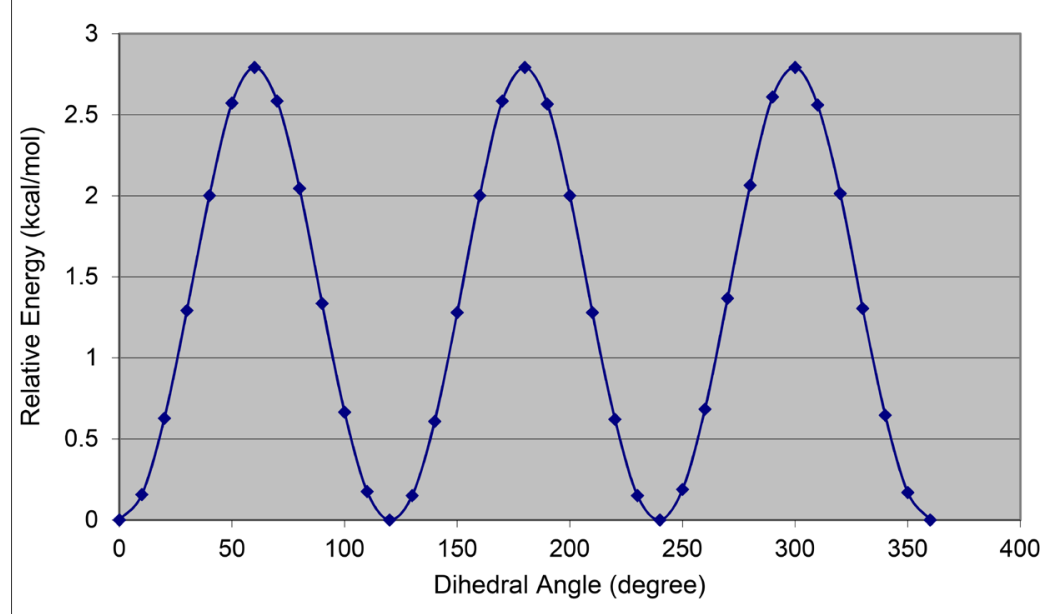

Figure S26. Potential energy profile of C-C and C-O internal rotors for $\mathrm{CH}_{3} \mathrm{CHFO} \bullet$.The solid lines indicate Fourier series expansion. 

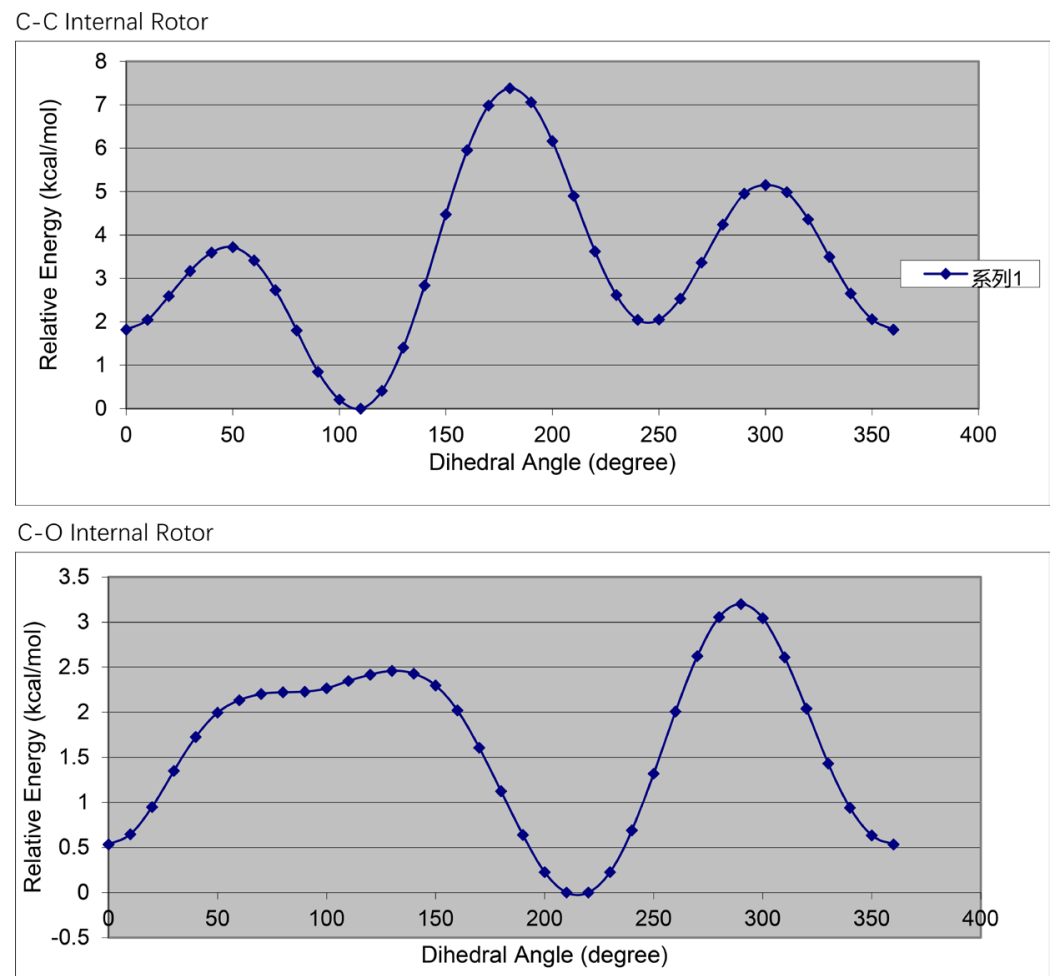

Figure S27. Potential energy profile of C-C and C-O internal rotors for $\mathrm{CH}_{2} \mathrm{FCHFOH}$. The solid lines indicate Fourier series expansion.
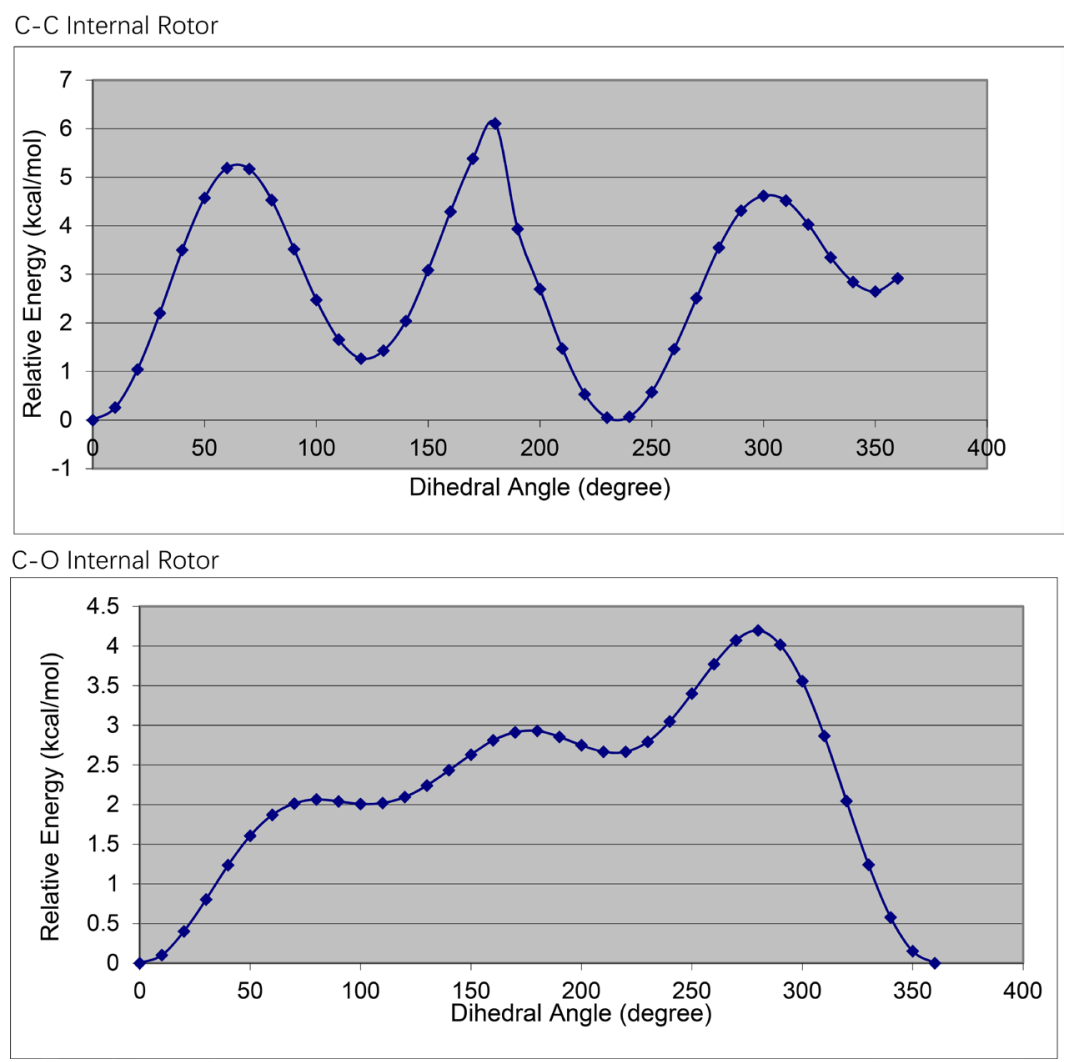

Figure S28. Potential energy profile of C-C and C-O internal rotors for $\mathrm{CF}_{2} \mathrm{HCH}_{2} \mathrm{OH}$. The solid lines indicate Fourier series expansion. 


\section{C-C Internal Rotor}
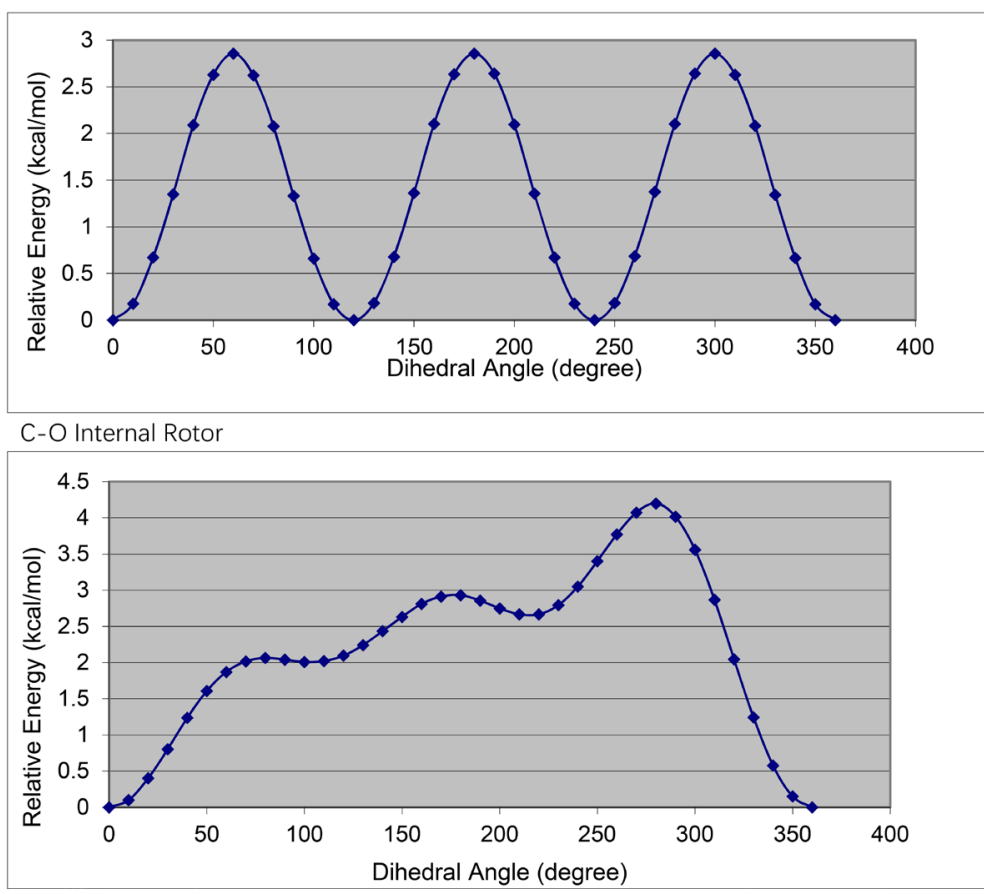

Figure S29. Potential energy profile of C-C and C-O internal rotors for $\mathrm{CH}_{3} \mathrm{CF}_{2} \mathrm{OH}$. The solid lines indicate Fourier series expansion.
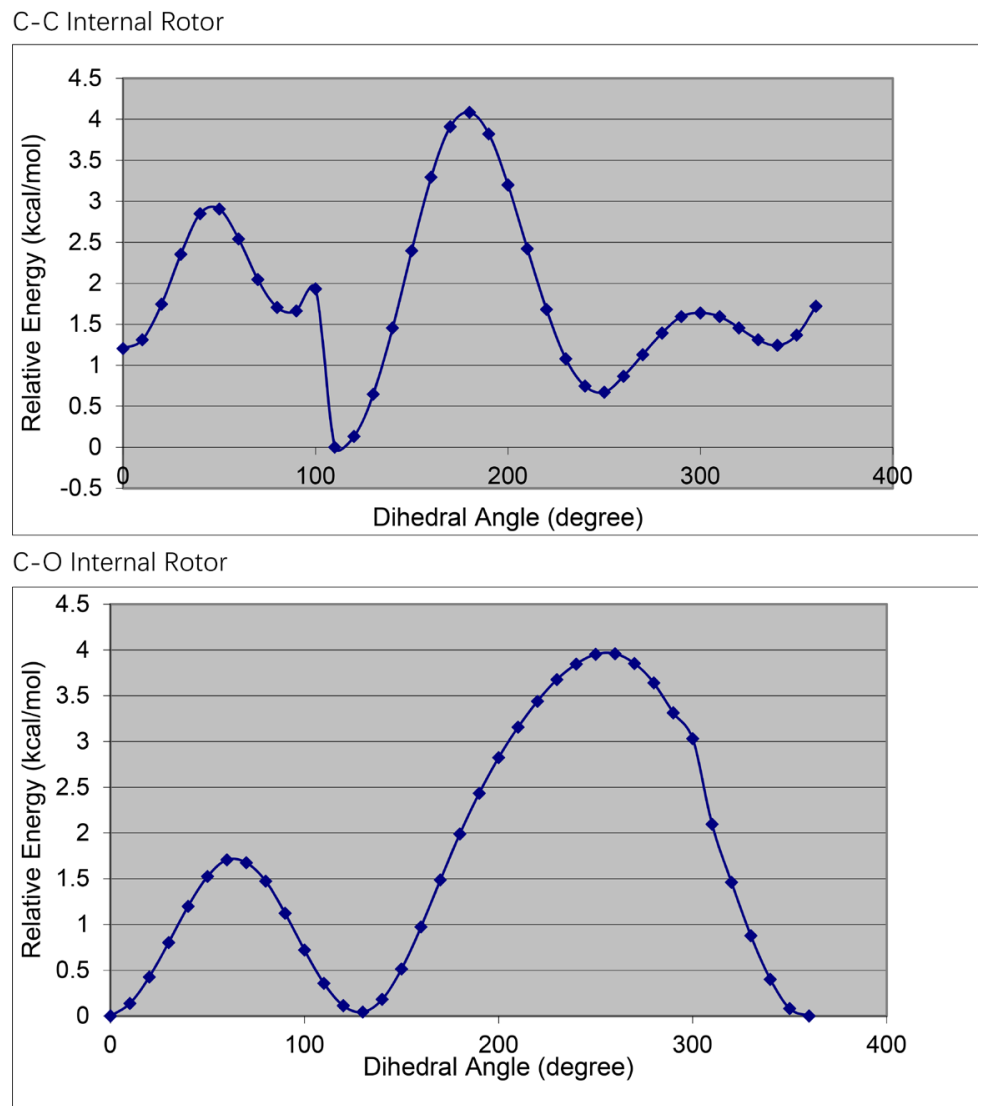

Figure S30. Potential energy profile of C-C and C-O internal rotors for $\mathrm{CH}_{2} \mathrm{FC} \cdot \mathrm{FOH}$. The solid lines indicate Fourier series expansion. 

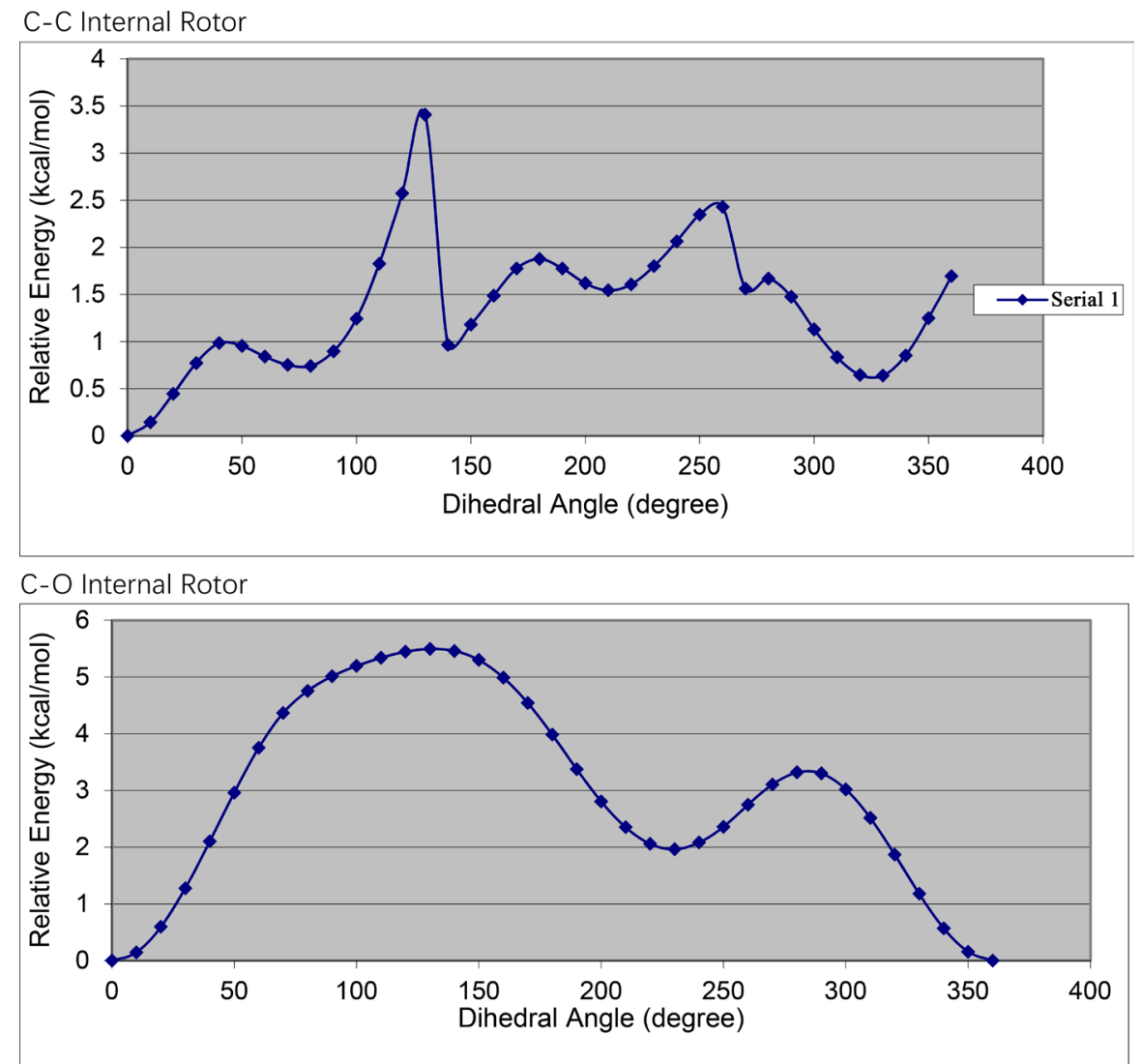

Figure S31. Potential energy profile of C-C and C-O internal rotors for C.HFCFHOH. The solid lines indicate Fourier series expansion.

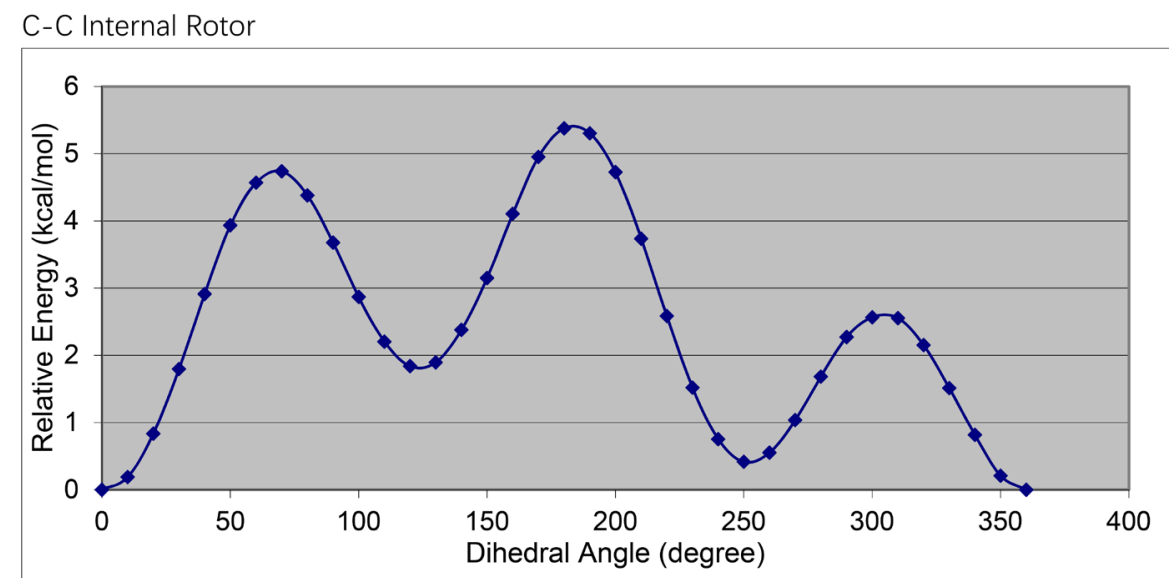

Figure S32. Potential energy profile of C-C and C-O internal rotors for $\mathrm{CH}_{2} \mathrm{FCHFO} \bullet$. The solid lines indicate Fourier series expansion. 

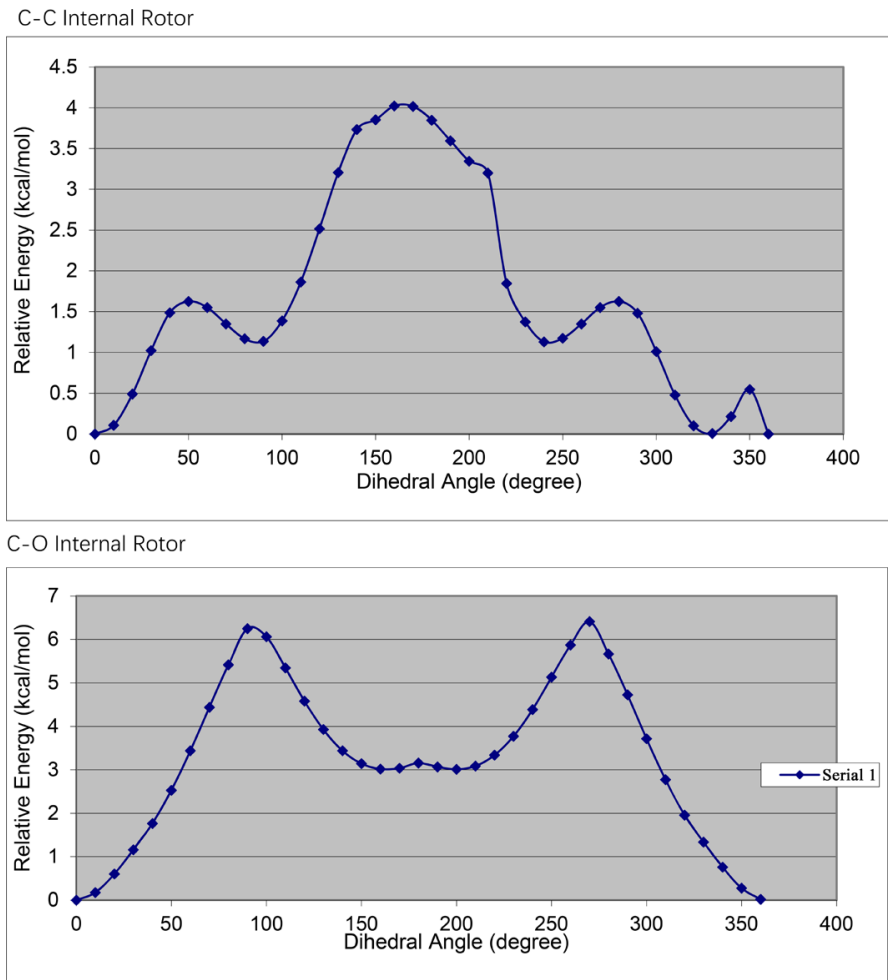

Figure S33. Potential energy profile of C-C and C-O internal rotors for $\mathrm{CHF}_{2} \mathrm{C} \cdot \mathrm{HOH}$. The solid lines indicate Fourier series expansion.
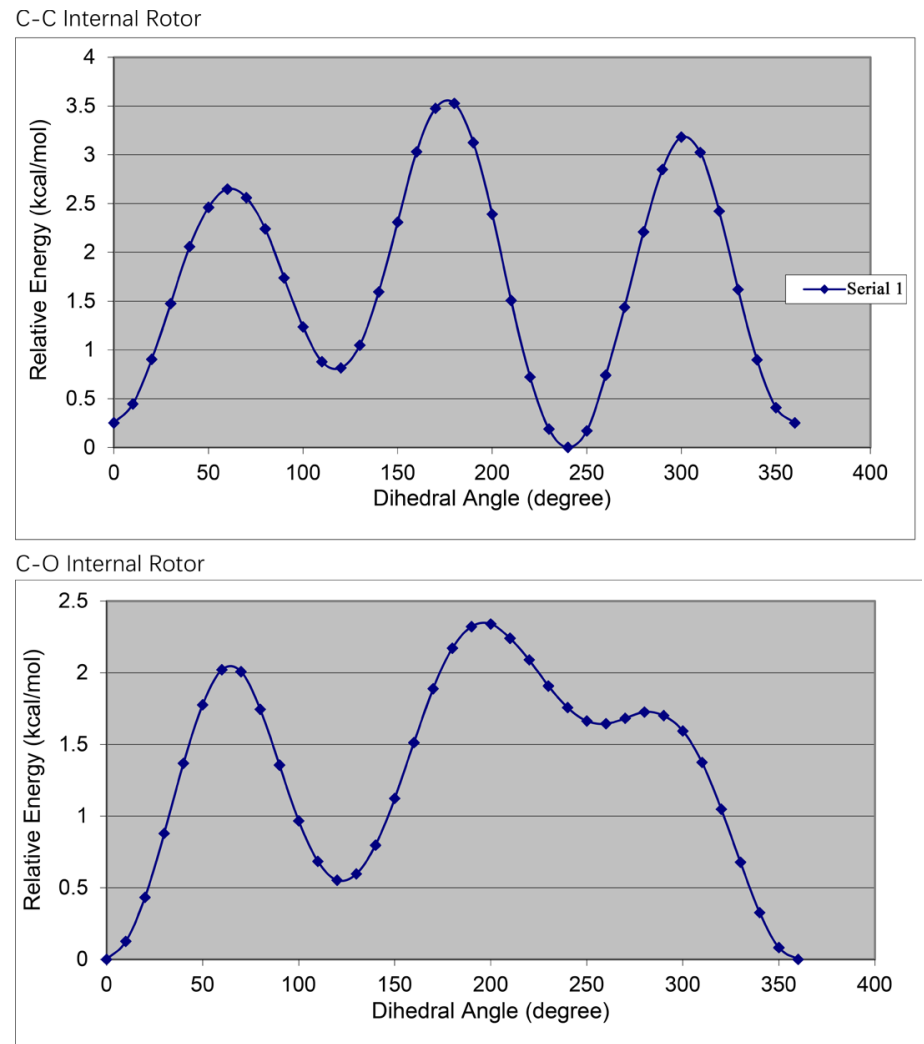

Figure S34. Potential energy profile of $\mathrm{C}-\mathrm{C}$ and $\mathrm{C}-\mathrm{O}$ internal rotors for $\mathrm{C} \cdot \mathrm{F}_{2} \mathrm{CH}_{2} \mathrm{OH}$. The solid lines indicate Fourier series expansion. 


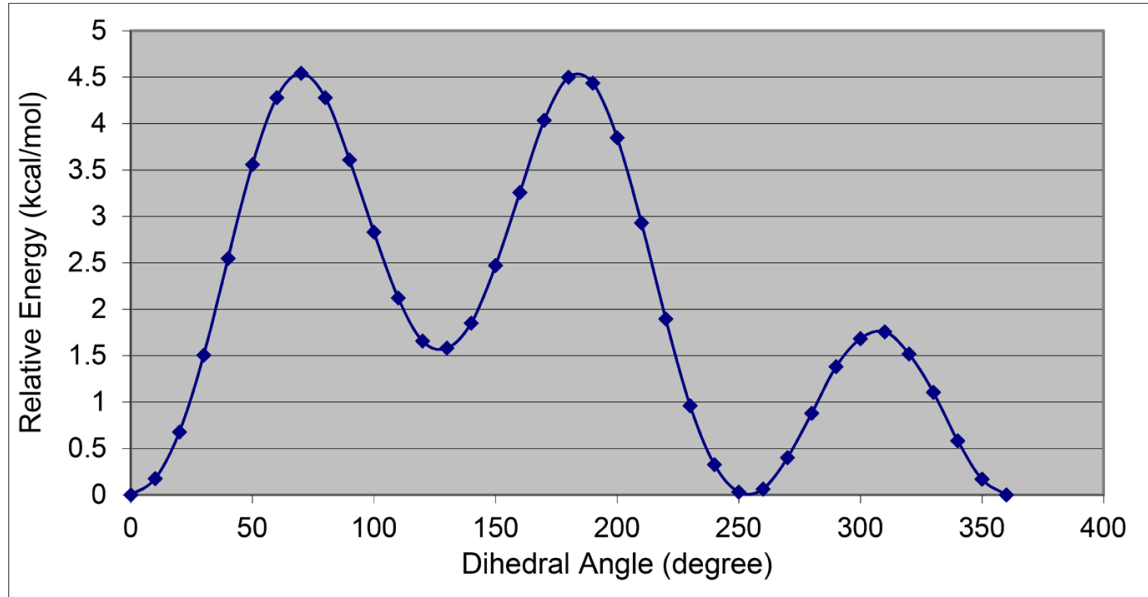

Figure S35. Potential energy profile of C-C and C-O internal rotors for $\mathrm{CHF}_{2} \mathrm{CH}_{2} \mathrm{O} \bullet$. The solid lines indicate Fourier series expansion.

\section{C-C Internal Rotor}

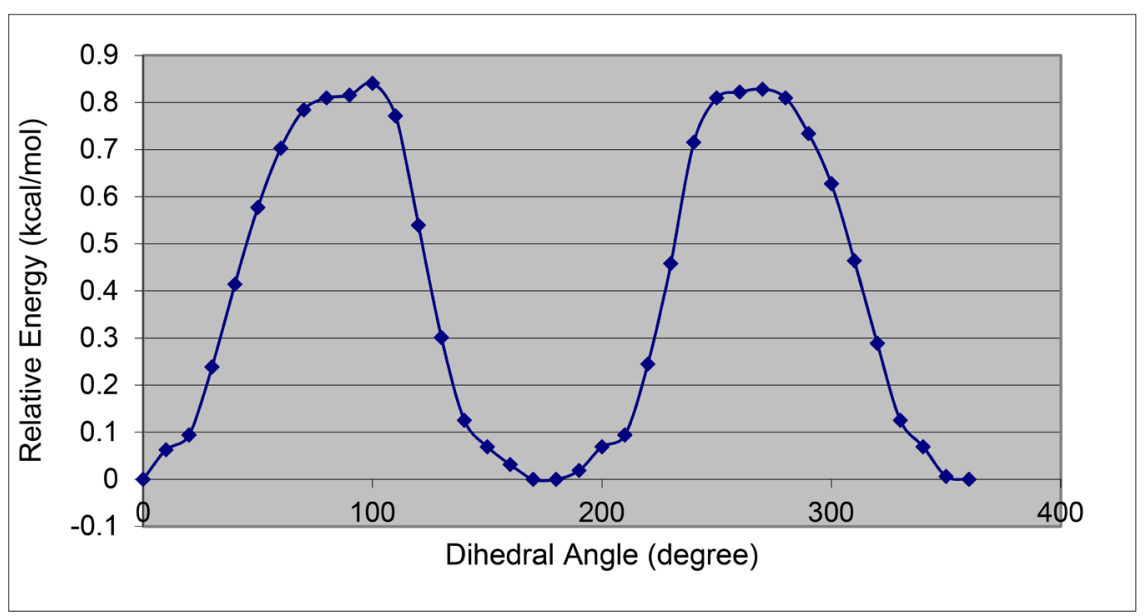

C-O Internal Rotor

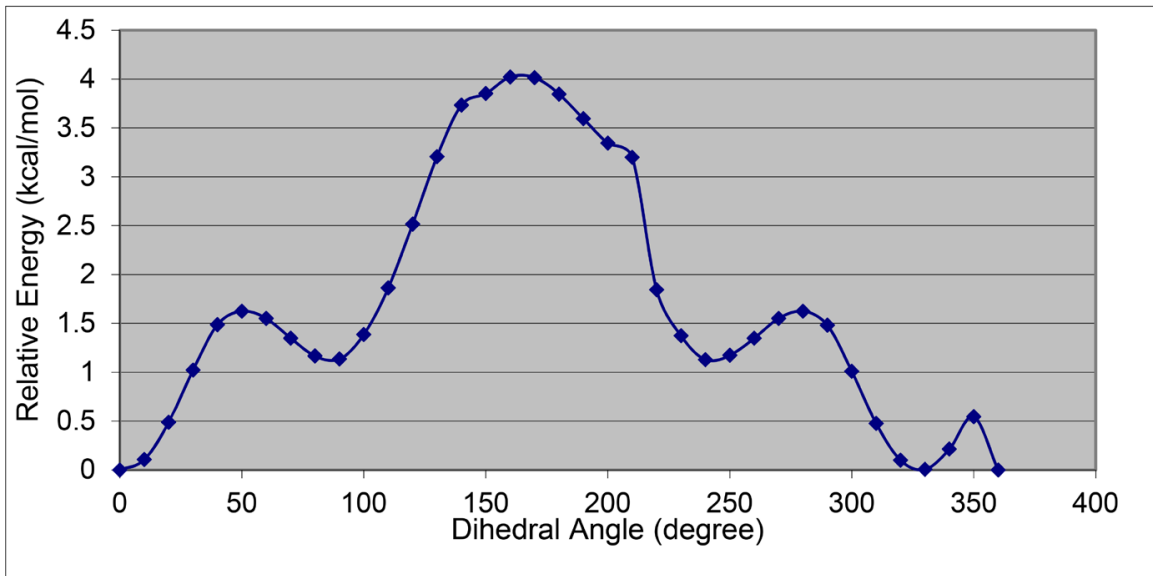

Figure S36. Potential energy profile of C-C and C-O internal rotors for $\mathrm{CH}_{2} \cdot \mathrm{CF}_{2} \mathrm{OH}$. The solid lines indicate Fourier series expansion. 


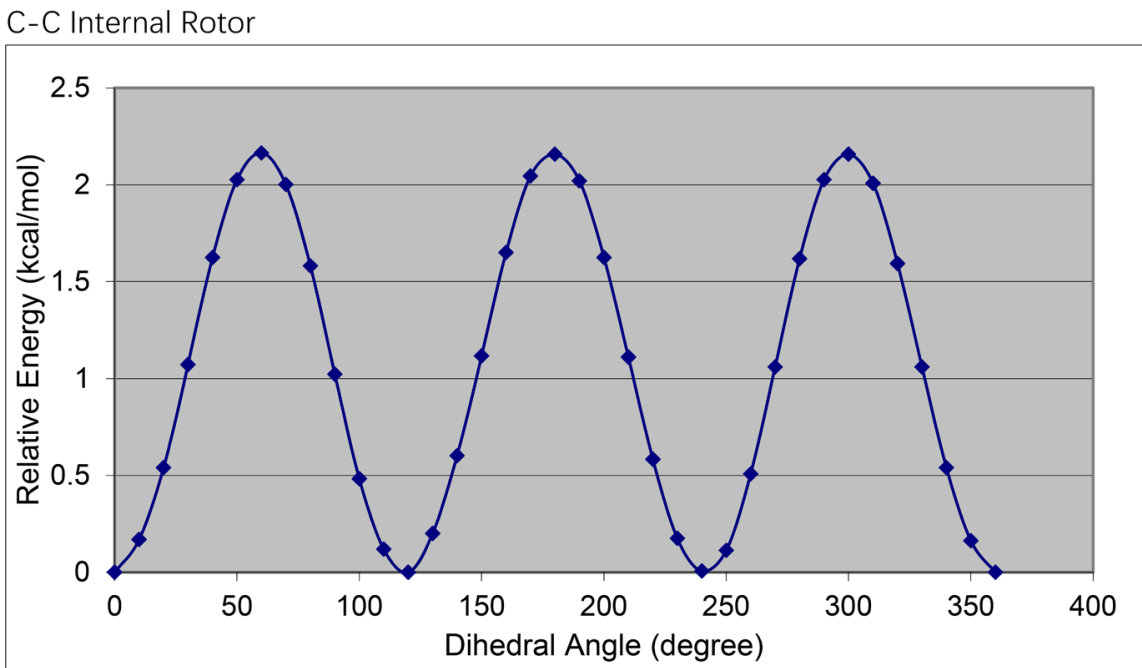

Figure S37. Potential energy profile of $\mathrm{C}-\mathrm{C}$ and $\mathrm{C}-\mathrm{O}$ internal rotors for $\mathrm{CH}_{3} \mathrm{CF}_{2} \mathrm{O} \bullet$. The solid lines indicate Fourier series expansion.

Table S4. The method of standard deviation and example calculation.

The standard deviation is calculated using the following formula (36):

$$
\sigma=\sqrt{\frac{1}{N} \sum_{i=1}^{N}\left(x_{i}-\mu\right)^{2}}
$$

where, $x_{i}$ is the mean; the average of the numbers, $\mu$ is the actual numbers to be calculated the standard deviation of, and

$$
\frac{1}{N} \sum_{i=1}^{N}\left(x_{i}-\mu\right)^{2}
$$

is the variance.

The standard deviation for standard enthalpy of formation for 1-fluroethanol is calculated to be \pm 0.1 . The calculation of standard deviation for this molecule is as follows:

$\mathrm{N}=2, \mu_{1}=-101.60, \mu_{2}=-101.86$

Step 1, $X_{i}=(-101.60-101.86) / 2=-101.73$

Step 2, $\left(X_{i}-\mu_{1}\right)=(-101.73+101.60)^{2}=0.0169$

$\left(X_{i}-\mu_{2}\right)=(-101.73+101.86)^{2}=0.0169$

Step 3, $1 / 2(0.0169+0.0169)=0.0169$

Step 4 , square root of $0.0169=0.13= \pm 0.1$

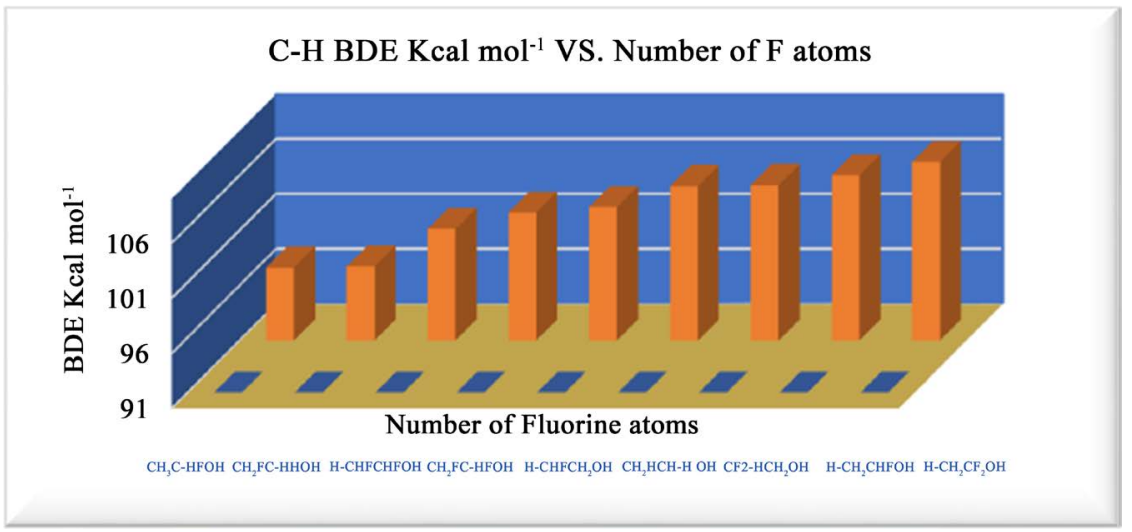

